\title{
FORSCHUNGSBERICHT 2017
}




\section{INHALTSVERZEICHNIS}

Forschungsbericht 2017

Grußwort der Präsidentin

Einführung in den Forschungsbericht

Übersicht über die laufenden Forschungsund Transferprojekte nach Forschungsbereichen der TH Wildau

FORSCHUNGSFELDER
9 Angewandte Biowissenschaften Applied Life Sciences

29 Informatik und Telematik Informatics and Telematics

43 Optische Technologien und Photonik Optical Technologies and Photonics

51 Produktion und Material Production and Materials

73 Verkehr und Logistik Transport and Logistics

85 Management und Recht Management and Law 


\section{GRUSSWORT \\ PROF. DR. RER. NAT. ULRIKE TIPPE}

Die Technische Hochschule Wildau im Land Brandenburg versteht sich als forschende Fachhochschule und ist als solche ein wichtiger Akteur in der Forschungslandschaft der Hauptstadtregion. Bereits zum neunten $\mathrm{Ma}$ legen wir mit diesem Forschungsbericht eine Übersicht über Projekte vor, die im Vorjahr bearbeitet worden sind. Das Spektrum reicht von kleineren Beratungs- und Forschungsprojekten bis hin zu mehrjährigen großen Verbundprojekten, die mit Partnern aus Wirtschaft und Wissenschaft bearbeitet werden. Die TH Wildau hat sich dabei auch überregional als kompetenter und verlässlicher Partner erwiesen.

Unsere Kooperationen in Forschung und Entwicklung verbinden unsere Hochschule sowohl mit international tätigen Großunternehmen der Industrie als auch mit innovativen kleinen und mittleren Unternehmen. Dabei steht die Anwendungsorientierung im Vordergrund. So konnten im Jahr 2017 beispielsweise acht Vorhaben im Zentralen Innovationsprogramm des Mittelstands (ZIM) des Bundesministeriums für Wirtschaft und Technologie erfolgreich eingereicht werden.
Ein besonderer Erfolg im Jahr 2017 war sicherlich die positive Bewertung des gemeinsam mit der BTU CottbusSenftenberg eingereichten Antrags "Innovation Hub 13 - Fast track to transfer" im Rahmen der Ausschreibung "Innovative Hochschule" des Bundesministeriums für Bildung und Forschung. Im Zeitraum von 2018 bis 2022 werden beide Hochschulen zusammen mit unseren Partnern aus Wirtschaft, Wissenschaft und Gesellschaft neue Wege im Bereich ,Transfer' gehen und so neue Impulse für die regionale Entwicklung in SüdostBrandenburg und Nordsachsen setzen.

Die erfolgreiche Projektarbeit ist ohne wirtschaftliche und wissenschaftliche Kooperationen nicht möglich. In den kommenden Jahren werden wir diese Partnerschaften weiter pflegen und ausbauen und dabei noch intensiver mit der Zivilgesellschaft in den Austausch kommen. Dazu bauen wir derzeit beispielsweise ein Bürgerpanel auf, das im Laufe des Jahres 2018 seine Arbeit aufnehmen wird.

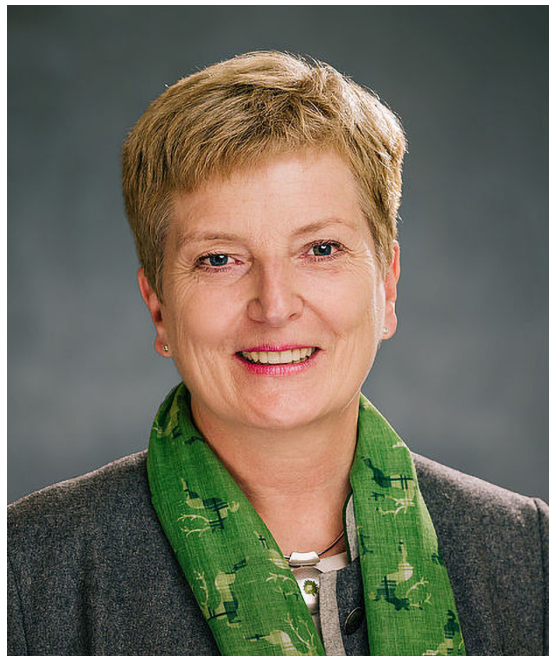

Ich lade Sie herzlich zur Lektüre des vorliegenden Forschungsberichts ein, in dem Sie sich über die „Forschungsbilanz 2017“ unserer Hochschule informieren können.

Alle Projekte finden Sie auch in der Projektdatenbank auf den Internetseiten der TH Wildau (https://www.th-wildau. de/forschung-transfer/projekte-publikationen-und-patente/).
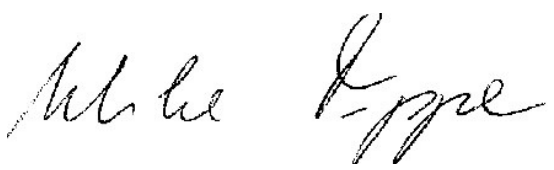

Prof. Dr. rer. nat. Ulrike Tippe Präsidentin der TH Wildau 


\section{EINLEITUNG}

\section{PROF. DR. RALF VANDENHOUTEN}

Angewandte Forschung und Entwicklung prägen den Charakter der $\mathrm{TH}$ Wildau, und Partner aus Wirtschaft, Politik und Verwaltung schätzen die Hochschule als verlässlichen Akteur. Im Jahr 2017 konnte die TH Wildau ihren erfolgreichen Weg weiterhin fortsetzen.

Drittmittel sind ein Indikator für erfolgreiche Forschungs- und Entwicklungsaktivitäten. Im Jahr 2017 lag das Drittmittelaufkommen der TH Wildau bei gut 11,04 Mio. Euro. Durch die eingeworbenen Drittmittel erhöhte sich der durch die Grundfinanzierung gedeckte Haushalt der Hochschule um fast zwei Drittel. Das ist bundesweit erneut ein Spitzenwert. Die Drittmitteleinnahmen lagen in den vergangenen 15 Jahren bei durchschnittlich 7,79 Mio. Euro, wobei es fast durchgängig einen Anstieg der Einnahmen pro Jahr gab. So konnte die Hochschule innerhalb der letzten fünf Jahre Drittmitteleinnahmen von durchschnittlich 11,37 Mio. Euro jährlich einwerben.

Die TH Wildau hat in den vergangenen Jahren die Interdisziplinarität in der Forschung auf verschiedene Weise gestärkt. Als ein Wegbereiter für disziplinübergreifende Projekte und Vorhaben hat die Digitalisierung in den vergangenen Jahren für die $\mathrm{TH}$ Wildau an Bedeutung gewonnen. Die moderne Arbeits- und Lebenswelt zeichnet sich dadurch aus, dass zunehmend Prozesse miteinander verknüpft und automatisiert werden. Die Informations- und Kommunikationstechnologien (IKT) sind der Treiber dieser Entwicklung. Die Verknüpfung und Automatisierung von Prozessen verschiedener Gewerke mittels IKT wird als digitale Integration verstanden. Sowohl die Hochschule als auch ihre Kooperationspartner stehen in diesem Bereich vor großen Herausforderungen. Für die TH Wildau ist es daher sinnvoll, diese Herausforde- rungen proaktiv anzugehen und Entwicklungen mitzugestalten.

Seit 2017 ist die TH Wildau beispielsweise Partner in zwei vom Bundesministerium für Wirtschaft und Energie geförderten Mittelstand 4.0-Kompetenzzentren. Das Mittelstand-4.0Kompetenzzentrum IT-Wirtschaft hat das Ziel, kleinen und mittleren ITUnternehmen vor Ort bei der Bewältigung der Herausforderungen der Digitalisierung zu helfen. Neben dem Konsortialführer - dem Bundesverband IT-Mittelstand e. V. - sind die TH Wildau und die TH Brandenburg Projektpartner. Die TH Wildau ist mit ihren Forschungsgruppen Innovations- und Regionalforschung und Rechtliche Rahmenbedingungen in dem Projekt vertreten. Im Mittelstand-4.0-Kompetenzzentrum Cottbus, welches von der BTU Cottbus-Senftenberg koordiniert wird, bringt die TH Wildau durch ihre Forschungsgruppen iC3@Smart Production und Sichere Objektidentitäten ihre Expertise ein. Im Zentrum des Vorhabens stehen Unternehmen des Mittelstandes und deren Mitarbeiter und Mitarbeiterinnen, die auf die verschiedenen, modularen Angebote des Kompetenzzentrums zurückgreifen können, um die digitale Transformation ihres Unternehmens weiter voranzutreiben.

Zudem ist die TH Wildau seit Ende 2017 als Testumgebung auf der Plattform i4KMU, der nationalen Kontaktund Koordinierungsstelle für I-4.0Testumgebungen des Bundesministeriums für Bildung und Forschung (BMBF), verzeichnet. Mit ihren Infrastrukturen und Kompetenzen in den Bereichen Automatisierung, Produktund Softwareentwicklung präsentiert die Hochschule Unternehmen und weiteren interessierten Partnern dabei nicht nur ein einzelnes Labor, sondern ein interdisziplinäres, fachbereichsübergreifendes Angebot.

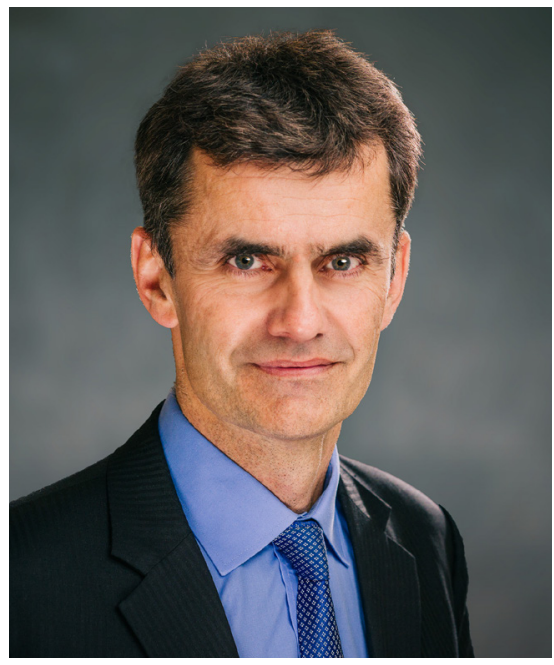

Viele Forschungsarbeiten und Projekte befassen sich in ihrem Kern bereits mit der digitalen Integration. In dieser Ausgabe des Forschungsberichts kennzeichnen wir diese Vorhaben wieder gesondert mit DI, um Ihnen eine Übersicht über die Spannweite der digitalen Integration zu geben.

Forschung bildet auch einen elementaren Bestandteil der praxisbezogenen Lehre, die ein Markenzeichen unserer Ausbildung ist. Für uns bilden Forschung und Lehre eine Einheit. Denn praxisorientierte Lehre muss sich durch die Forschungstätigkeit der Kolleginnen und Kollegen immer wieder erneuern und am aktuellen Stand der Wissenschaft ausrichten. Die anwendungsorientierten Forschungsprojekte bereichern nicht nur Lehre und Studium durch anspruchsvolle Themen. Sie bieten Studierenden auch die Möglichkeit zur aktiven Mitwirkung bereits während des Studiums und stellen die zentrale Finanzquelle für die praxisnahe Forschung der Zukunft dar. Wie die Lehre gehört an der TH Wildau zur Forschung auch der Wissens- und Technologietransfer. Angewandte Forschung und Entwicklung sind kein Selbstzweck, sondern sie leisten einen Beitrag zu unserem gesellschaftlichen Wohlstand. 


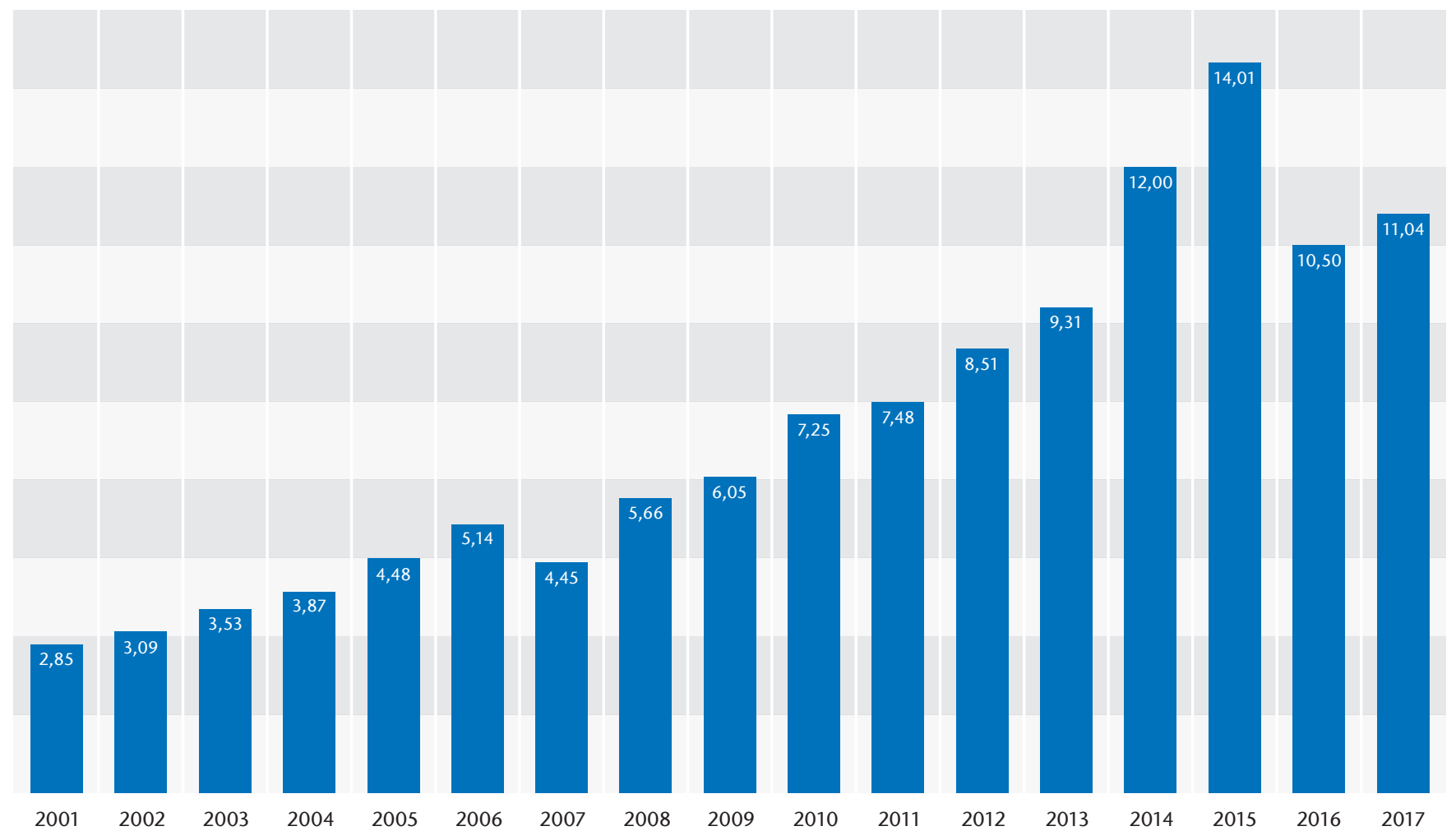

Ein Meilenstein für die zukünftige Weiterentwicklung des Wissens- und Technologietransfers war der erfolgreich eingereichte Antrag der $\mathrm{TH}$ Wildau mit der BTU Cottbus-Senftenberg im BMBF-Wettbewerb „Innovative Hochschule“. Im Vorhaben „Innovation Hub 13 - Fast track to transfer " werden die beiden Hochschulen ab 2018 einen Innovation Hub aufbauen und betreiben. Der Innovation Hub integriert und organisiert die verschiedenen Innovationsakteure der Region mit deren Wissen und Ressourcen, technischen Infrastrukturen sowie Methoden und Interessen, wobei die zusammengeführten Hochschulkompetenzen sein Aktivitätszentrum bilden. Mit dem Innovation Hub werden Voraussetzungen geschaffen, akteursübergreifend Problemlösungen für zentrale gesellschaftliche Herausforderungen der Region Südostbrandenburg zu entwickeln.

Mit der verstärkten Öffnung für Interessenten außerhalb der Hochschule festigt die TH Wildau entsprechend ihrem Leitbild ihre Rolle als gefragter und zuverlässiger Partner der Wirtschaft und als Wachstumsmotor der
Region. Vor allem kleine und mittlere Unternehmen profitieren von der angewandten Forschung, können eigene Forschungskompetenzen entwickeln und so ihre Wettbewerbsfähigkeit sichern.

Das zentrale An-Institut Technologieund Weiterbildungszentrum an der TH Wildau e. V. (TWZ e. V.) hat sich als Dach für die dort ansässigen Institute bewährt. Die Institute des TWZ e. V. führen vorwiegend Auftragsforschung und -entwicklung sowie Dienstleistungsprojekte durch, z. B. im Weiterbildungsbereich.

Die technische Ausstattung der Hochschule ist hervorragend, weil die $\mathrm{TH}$ Wildau in den vergangenen zwei Dekaden überdurchschnittlich erfolgreich Infrastrukturmittel einwerben konnte. Gerade die geräteintensiven Forschungsschwerpunkte, wie zum Beispiel Angewandte Biowissenschaften, Optische Technologien/ Photonik oder Informatik/Telematik können eine national wie international wettbewerbsfähige Infrastruktur vorweisen. Dadurch existieren Rahmenbedingungen, in denen exzellen- te Forschungs- und Entwicklungsleistungen möglich sind. Die Hochschule zeichnet ein forschungsfreundliches Klima aus, das den Forschergeist der Kolleginnen und Kollegen inspiriert und auch zunehmend zu disziplinübergreifenden Forschungsprojekten führt.

Der vorliegende Forschungsbericht 2017 gibt eine Übersicht über die laufenden Projekte aus dem Jahr 2017. Ausgewählte Projekte werden ausführlicher dargestellt. Die Projekte sind nach Forschungsfeldern geordnet. Ich wünsche Ihnen eine anregende Lektüre. Wir freuen uns über Ihre Kontaktaufnahme - wenn Sie Fragen oder Anregungen haben oder wenn Sie sich für eine Zusammenarbeit in bestimmten Bereichen interessieren.

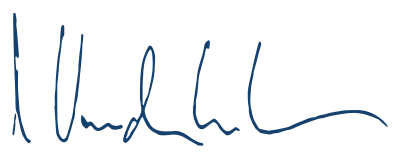

\section{Prof. Dr. Ralf Vandenhouten}

Vizepräsident für Forschung und Transfer 


\section{ÜBERSICHT ÜBER DIE LAUFENDEN FORSCHUNGS- UND TRANSFERPROJEKTE 2017}




\section{Angewandte Biowissenschaften Applied Life Sciences}




\title{
Photobioelektroden auf der Basis von dreidimensionalen Elektrodenstrukturen
}

\author{
Prof. Dr. Fred Lisdat
}

$\begin{array}{ll}\text { PROJEKTMITARBEITER(INNEN) } & \text { Dipl.-Biochem. D. Ciornii } \\ \text { KOOPERATIONSPARTNER } & \text { Dr. N. Nioradze, Prof. D. Khoshtariya (Javakhishvili Tbilisi State University) } \\ \text { PROJEKTVOLUMEN } & 10.000 € \\ \text { MITTELGEBER } & \text { DAAD | Rustaveli National Science Foundation Georgia } \\ \text { LAUFZEIT } & 6 / 2017-11 / 2017\end{array}$

Die schnelle Elektrochemie von Redoxproteinen wie Cytochrom c bietet die Möglichkeit, auch komplex aufgebaute, katalytisch aktive Proteine mit Elektrodenoberflächen zu verbinden. Hier wird auf die natürliche ShuttleFunktion dieses Hämproteins zurückgegriffen.

Ein neueres Anwendungsgebiet dieser Redoxproteine sind photokatalytisch aktive Elektroden, die für die Lichtzu-Strom- bzw. Licht-zu-WertstoffUmwandlung interessant sind. Hier konnten in der AG Biosystemtechnik bereits mehrere leistungsfähige Elektrodensysteme mit dem biologischen Superkomplex Photosystem I aufgebaut werden. Dreidimensionale BasisElektroden sind hierbei besonders wertvoll, da dadurch die Effektivität der Photostromgenerierung deutlich gesteigert werden kann.

Im Rahmen des gemeinsamen Forschungsvorhabens mit dem georgischen Partner wurden nun verschiedene Ansätze untersucht, wie solche strukturierten Elektrodenoberflächen für die Photobioelektrochemie aufgebaut werden können. Hierfür wurde ein Gastaufenthalt von Dr. N. Nioradze an der TH Wildau realisiert. Im Fokus der Arbeiten stand die Methode des Elektrospinnings mit anschließender Kalzinierung. Beim Elektrospinnen wird ein Hochspannungsfeld dafür genutzt, aus einer Polymerlösung eine faserartige Struktur aufzubauen. Dabei können vorteilhaft Nanostrukturen, wie zum Beispiel aus leitfähigen Indium/Zinnoxid-Nanopartikeln, integriert werden.

An diesen Oberflächen musste zunächst die Elektrochemie des Shuttlemoleküls Cytochrom c und dann die Interaktion mit dem Photosystem I charakterisiert werden. Hier verbinden sich die Expertisen der beiden Partner. Final sind schließlich neue Photobioelektroden konstruiert worden, die eine effektive Umwandlung von Licht in Strom ermöglichen. Dabei kann die Photostromproduktion durch die Spinnzeit in der Herstellung in einem weiten Bereich eingestellt werden.

KONTAKT

fred.lisdat@th-wildau.de

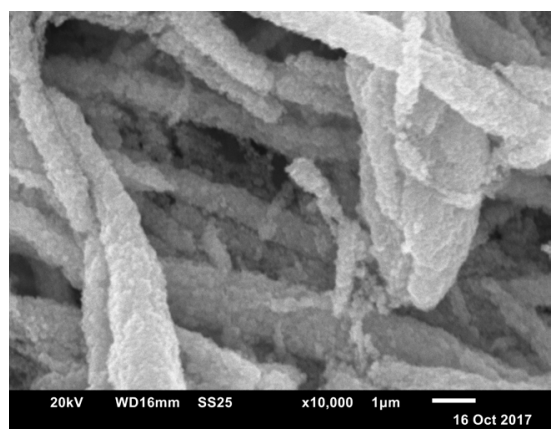

Abb. 1) Die Abbildung zeigt eine elektronenmikroskopische Aufnahme einer Elektrodenstruktur, die durch Elektrospinnen von Indium/Zinnoxid-Nanopartikeln in einer Polymerlösung mit anschließender Temperierung erzeugt wurde. Sie bietet eine erhöhte Oberfläche für die Anbindung und Kontaktierung der Biomoleküle Photosystem I und Cytochrom c.

Der weiße Strich zeigt die Dimension von einem Mikrometer $(10-6 \mathrm{~m})$. 


\title{
TABELLARISCHE ÜBERSICHT LAUFENDER PROJEKTE
}

\author{
MiniSeq: Low Cost Sequencing \\ \begin{tabular}{l|l} 
Projektleiter Prof. Dr. rer. nat. Peter Beyerlein & Pren
\end{tabular} \\ Mittelgeber Land Brandenburg | MWFK | EFRE | InfraFEI \\ Projektvolumen $\quad 90.000 €$ \\ Laufzeit $\quad$ 02/2017-01/2018 \\ Beschaffung eines Low-Cost Sequencers für die Entwicklung von alltagstauglichen Sequenzierungsprotokollen und \\ Sequenzierungs-Apps. Die zunehmende Reduktion von Chemiekosten und Gerätekosten erlauben unter Auswertung \\ bereits vorhandener BigData (und umfangreicher Erfahrung mit Forschungsstudien aus vergangenen Jahren) die F\&E an \\ Apps, auf Basis von Sequenzierungskleingeräten, mit denen alltägliche Sequenzierungs-Aufgaben gelöst werden können.
}

\section{Philips-2017-2: Prostate Cancer Cell Composition Analyses}

Projektleiter

Kooperationspartner

Mittelgeber

Laufzeit
Prof. Dr. rer. nat. Peter Beyerlein

\section{Wirtschaft}

Philips Research

$12 / 2017-06 / 2018$

Fortsetzungsprojekt: Bioinformatischen Analyse der Zelltypzusammensetzung von Prostata Tumoren von 500 klinischen Tumor Samples, Fokus auf Genexpression, Mutation, Gen-Fusion, Zell-Komposition

\section{Philips-NGS-2016: Sequenz-Datenanalyse Prostatakrebs}

Projektleiter

Kooperationspartner

Mittelgeber

Laufzeit
Prof. Dr. rer. nat. Peter Beyerlein

Wirtschaft

Philips Electronics

10/2016-12/2017

Die Philips Forschung beauftragt die Forschungsgruppe Diagnostische Bioinformatik von Prof. Dr. Beyerlein mit der Datenanalyse von ca. 500 Patientenprobenzum Aufbau von diagnostischen und therapiebegleitenden Tests. Der Fokus liegt dabei auf Transkritomen, Genomen und Fusionen. 
8sens: Universelles Lesegerät für Schnelltestsysteme (IBA-IVD)

Projektleiter

Prof. Dr. rer. nat. Andreas Foitzik

Kooperationspartner 8sens.biognostic $\mathrm{GmbH}$

Mittelgeber

VDI | VDE

Laufzeit

$01 / 2016-12 / 2017$

Projektvolumen

$153.248 €$

Projekthomepage

www.th-wildau.de/im-studium/fachbereiche/igw/igw-studiengaenge/bb-forschung/mikrosys-

temtechnik0/mst-forschung.html

Ziel ist die Entwicklung eines Low-Cost-Messsystems für Smartphones zur qualitativen und quantitativen Bestimmung von biologischen Analyten mittels Lateral Flow Assay-Teststreifen. Das Messsystem soll aus einem blickdichten Messgehäuse mit einer Teststreifenaufnahme, einer Weißlichtquelle und einer Fluoreszenz-Lichtquelle bestehen. Kernstück des Gehäuses ist eine Smartphoneaufname im Messgehäuse sowie einer App für Smartphones zur visuellen Aufnahme und Auswertung der Testergebnisse von Lateral.

\section{WeFilTi: 96-Well Probenfiltrationssystem für die Auftrennung von biologischen Probenbestandteilen}

Projektleiter

Kooperationspartner

Mittelgeber

Projektvolumen

Laufzeit
Prof. Dr. rer. nat. Andreas Foitzik

bbi-biotech $\mathrm{GmbH}$

Bund | BMWi | ZIM Kooperationsprojekt

$189.140 €$

07/2016-06/2018

Im Rahmen des Projektvorhabens wird durch die beteiligten Partner, die TH Wildau und die bbi-biotech GmbH, die Realisierung eines neuartigen Filtersystems auf Grundlage des 96-Well Mikrotiterformats angestrebt. Ziel ist es, beliebige zellhaltige Proben aus Bioreaktoren fluidisch aufzutrennen und die resultierenden Filtrate über einen begrenzten Zeitraum zu lagern. Durch die Projektpartner wird hierbei die Technik der Druckfiltration favorisiert.

Bilderkennungsstudie für landwirtschaftlich genutzte Flächen

\begin{tabular}{|l|l|}
\hline Projektleiter & Prof. Dr. rer. nat. Andreas \\
\hline Mitarbeiter & Thilo Liebscher \\
\hline Kooperationspartner & HORATIO GmbH \\
\hline Mittelgeber & FUE / Transferbonus klein \\
\hline Projektvolumen & $3.000 €$ \\
\hline Laufzeit & $04 / 2017-07 / 2017$ \\
\hline
\end{tabular}

Die automatisierte Erkennung von landwirtschaftlichen Flächen und deren Beurteilung nach ökonomischen und ökologischen Kriterien mit Hilfe einer mobilen Anwendung. 
Designstudie für ein Blutentnahmesystem

Projektleiter

Mittelgeber

Laufzeit
Prof. Dr. rer. nat. Andreas Foitzik

Midge medical UG

08/2016-01/2017

Die Entwicklung eines mobilen Sytems zur Entnahme von Blut durch einen einfachen Auslösemechanismus ist Ziel dieses Projektes. Abgenommenes Blut wird in ein Reservoir geleitet und dort für weitere analytische Schritte vorbereitet.

\section{Einweg Kühlwärmesystem}

Projektleiter

Prof. Dr. rer. nat. Andreas Foitzik

Mitarbeiter

Thilo Liebscher

Mittelgeber

Torsten Müller

Laufzeit

$11 / 2016-12 / 2018$

Dieses Projekt beinhaltet eine Konstruktionsstudie eines Einweg Heiz- und Kühlsystems für eine Herz-Lungen-Maschine auf Basis von 3D CAD-Daten zur Visualisierung des Wärme- und Flusskonzeptes.

Entwicklung eines innovativen Dispensers für Rein- und Reinstwassersysteme

Projektleiter

Prof. Dr. rer. nat. Andreas Foitzik

Mitarbeiter

Thilo Liebscher

Kooperationspartner MembraPure GmbH

Mittelgeber

FuE | BIG-FuE

Projektvolumen $49.454 €$

Laufzeit

$12 / 2016-12 / 2018$

Entwicklung eines neuartigen hochreinen Entnahmearms (auch Dispenser genannt) für Wasserreinigungssysteme mit Hilfe verbunden durch die Bluetooth-Technologie. Dieser Entnahmearm wird benötigt, um die Entnahme von der Wasseraufbereitung zu entkoppeln und damit Platz zu sparen im Gerät. 
HFM-IC: Hochdruckfiltereinsatzmagazin für IC-Systeme

Projektleiter

HFM-IC: Hochdruckfiltereinsatzmagazin für IC-Systeme

Mitarbeiter

Thilo Liebscher

Kooperationspartner

MembraPure GmbH

Mittelgeber

Bund | BMWi | ZIM Kooperationsprojekt

Projektvolumen

$189.071 €$

Laufzeit

07/2017-06/2019

Gegenstand des kooperativen Projektvorhabens ist die Entwicklung eines modularen Filtereinsatzmagazins zur Anwendung in IC-Analyse-Systemen. Dieses System soll in der Lage sein, Proben rückstandslos und innerhalb des Druckbereiches zu filtrieren. Hierbei darf das Filtrationssystem unter keinen Umständen die Analysestrecke negativ beeinflussen. Entsprechend ist die Integration ohne Druckverlust bzw. Austreten von Flüssigkeit zu gewährleisten.

\section{ISKS: Innovative Systemlösung für Kugellager durch Steckverbindungen}

Projektleiter

Mitarbeiter

Kooperationspartner

Mittelgeber

Projektvolumen

Laufzeit
Prof. Dr. rer. nat. Andreas Foitzik

\section{Thilo Liebscher}

Metallbau Glawion GmbH

Bund | BMWi | ZIM Kooperationsprojekt

$189.945 €$

06/2017-11/2018

Das Ziel des Projekts ist es, einen Käfig in vorgewalzte Segmente aufzuteilen, welche beim Kunden, auch durch den Kunden, zu einem Käfig zusammengefügt werden können. Die Verbindung soll aber nicht durch herkömmliche Schweißnähte erfolgen, sondern durch eine Verpressung. Dazu soll eine Steckverbindung entwickelt werden, mit welcher die einzelnen Segmente des Kugelkäfigs wie bei einem Puzzle zusammengefügt werden können.

MACIC - Mikro-Absorber für Combustion-Ionenchromatographie

\begin{tabular}{|l|l|}
\hline Projektleiter & Prof. Dr. rer. nat. Andreas Foitzik \\
\hline Mittelgeber & Bund / BMWi | ZIM \\
\hline Projektvolumen & $194.085 €$ \\
\hline Laufzeit & $05 / 2015-04 / 2017$ \\
\hline
\end{tabular}

Ziel des zu beantragenden Projekts ist die Entwicklung eines miniaturisierten und mikrostrukturierten Absorbers, der einer Verbrennungseinheit nachgestellt und einer Messeinheit für lonenchromatographie vorgeschaltet ist. Mit diesem Absorber sollen kleinste Volumina an Ausgangsstoffen einer dennoch präzisen chemischen Analyse für Halogene und Schwefel zugeführt werden. 
MBTK - Entwicklung des Prototyps eines vollautomatischen zellbiologischen Multi BenchTop

Projektleiter

Prof. Dr. rer. nat. Andreas Foitzik

Mittelgeber

Bund | BMWi | ZIM

Projektvolumen

$194.292 €$

Laufzeit

02/2015-01/2017

Das angestrebte Projekt verfolgt als Ziel die Realsisierung eines Multi BenchTop Kultivators (MBTK) mit integrierter Sensorik, einem Heiz- und Kühlkreislauf inklusive externen und internen Versorgunsmodulen. Dies soll letztlich die autarke und vor allem vollautomatische Kultivierung von (i) Organoiden, (ii) 3D-Zellkulturen ohne organähnliche Eigenschaften und (iii) 2D-Zellkulturen in Mikroreaktoren ( $\mu$ l-Volumina) ermöglichen.

Mechanische und medizinische Untersuchung anhand eines Funktionsmusters für ein neuartiges

Blutentnahmesystem

Projektleiter

Prof. Dr. rer. nat. Andreas Foitzik

Mittelgeber

Midge medical UG

Laufzeit

$11 / 2016-01 / 2017$

In diesem Projekt wird ein mobiles System zur Entnahme von Blut durch einen einfachen Auslösemechanismus entwickelt. Abgenommenes Blut wird in ein Reservoir geleitet und dort für weitere analytische Schritte vorbereitet.

Midge: Innovatives miniaturisiertes Blutabnahmesystem. Mechanisches Sicherheitssystem und Assemblierung

\begin{tabular}{|c|c|}
\hline Projektleiter & Prof. Dr. rer. nat. Andreas Foitzik \\
\hline Mitarbeiter & Thilo Liebscher \\
\hline Kooperationspartner & Meisterwerk Ventures GmbH \\
\hline Mittelgeber & Bund | BMWi | ZIM \\
\hline Projektvolumen & $189.976 €$ \\
\hline Laufzeit & $11 / 2017-11 / 2019$ \\
\hline
\end{tabular}


MNA: Mikro-Nadel-Array - Entwicklung von Abformwerkzeugen sowie Erarbeitung einer reproduzierbaren

kontinuierlichen Fertigung

\begin{tabular}{|c|c|}
\hline Projektleiter & Prof. Dr. rer. nat. Andreas Foitzik \\
\hline Mitarbeiter & Kai-Henning Lietzau \\
\hline Kooperationspartner & TheraKine BioDelivery GmbH \\
\hline Mittelgeber & Bund | BMWi | ZIM \\
\hline Projektvolumen & $189.430 €$ \\
\hline Laufzeit & 04/2017-10/2017 \\
\hline
\end{tabular}

PolyMed: Polymere für die Medizintechnik

\begin{tabular}{|c|c|}
\hline Projektleiter & Prof. Dr. rer. nat. Andreas Foitzik \\
\hline Mittelgeber & Bund | BMWi | ZIM \\
\hline Projektvolumen & $59.862 €$ \\
\hline Laufzeit & $03 / 2016-02 / 2018$ \\
\hline \multicolumn{2}{|c|}{$\begin{array}{l}\text { Es ist eine Innovationsplattform auf dem Gebiet der Medizintechnik, insbesonere im Bereich der Kunststoffverarbeitung } \\
\text { entstanden. Das strategische Ziel der im Netzwerk zusammengeschlossenen Unternehmen besteht in der Entwicklung, } \\
\text { Realisierung und Vermarktung innovativer Kunststoffprodukte sowie die Vernetzung dieser mit elektronischen Kompo- } \\
\text { nenten für die Medizinbranche, um langfristig die Innovationskraft der regionalen Wirtschaft zu stärken. Die Grundidee } \\
\text { besteht darin, Problemstellungen in Zusammenarbeit zu lösen. }\end{array}$} \\
\hline
\end{tabular}

agru Physics - physikalische Methoden für Nachhaltigkeit in Landwirtschaft und Umwelt

\begin{tabular}{|l|l|}
\hline $\begin{array}{l}\text { Projektleiter } \\
\text { Kooperationspartner }\end{array}$ & Prof. Dr. sc. hum. Marcus Frohme \\
\hline Mittelgeber & Bund | BMWi | ZIM Netzwerk \\
\hline Laufzeit & 04/2017-03/2018 \\
\hline
\end{tabular}

Das Netzwerk versteht sich als eine offene FuE-Plattform für innovative Unternehmen auf den Gebieten der Umwelt, Landwirtschaft und verarbeitenden Industrie landwirtschaftlicher Rohstoffe. 
BIOMES.world - wissenschaftliche Analyse der individuellen Mikrobiota

Projektleiter

Prof. Dr. sc. hum. Marcus Frohme

Mittelgeber

Bund | BMWi | ESF | EXIST

Projektvolumen

$150.600 €$

Laufzeit

$07 / 2017-06 / 2018$

Das Gründungsvorhaben baut eine onlinebasierte Produktdienstleistung zur Analyse der individuellen Mikrobiota auf. Dem Kunden wird per Webinterface sein mikrobielles Profil, bspw. aus dem Darm, erläutert und verständlich gemacht, es werden Hinweise zur Ernährung und Lebensführung gegeben und ggf. weitere Dienstleistungen oder Produkte angeboten.

\section{CyanoScreen - Entwicklung von Microcystin als Wirkstoffkandidaten gegen Tumore}

\begin{tabular}{l|l} 
Projektleiter & Prof. Dr. sc. hum. Marcus Frohme
\end{tabular}

Mittelgeber $\quad$ Bund | BMWi | ZIM

\begin{tabular}{l|l} 
Projektvolumen & $175.000 €$
\end{tabular}

Laufzeit $\quad$ 07/2015-09/2017

Cyanobakterien werden auf neue Microcystine (Toxine) im Hochdurchsatz untersucht. Hierzu wird eine an der TH Wildau entwickelte Screeningplattform weiterentwickelt und eingesetzt. Zusätzliche Variabilität wird über Fütterung gesonderter Aminosäuren und genetische Manipulation erzeugt. Ziel ist die Identifikation eines auf den OATP1B3-Transporter spezifischen Toxins für den potenziellen Einsatz als Tumortherapeutikum.

Digilog: Digitale und analoge Begleiter für eine alternde Bevölkerung

\begin{tabular}{l|l} 
Projektleiter Prof. Dr. sc. hum. Marcus Frohme & Prohe
\end{tabular}

Mittelgeber Land Brandenburg | MWFK | Gesundheitscampus

Projektvolumen $\quad 84.000 €$

Laufzeit

$03 / 2017-12 / 2018$

Die Vorsorge, Behandlung und Nachsorge von kranken Menschen und Risikozielgruppen sollen nicht an eine stationäre Einrichtung oder Praxis gebunden sein müssen, sondern um technische Leistungen und Prozesse in einer digitalen Welt ergänzt werden und damit den Verlust einer heimatnahen Versorgung ausgleichen. Ziel ist die Etablierung eines eHealthCenters von mHealth Daten aus „digitalen“ und „analogen“ Quellen und seine Vernetzung mit traditionellen Instanzen der regionalen Versorgungsstruktur. 
Projektleiter

Prof. Dr. sc. hum. Marcus Frohme

Mittelgeber

HS Diagnomics GmbH

Laufzeit

$10 / 2016-01 / 2017$

Im Rahmen des Projektes sollen bekannten Sequenzen der einzelnen Ketten von T-Zell Rezeptoren aus verschiedenen TZelllinien verknüpft werden. Die Verknüpfung soll in einem Rasterformat und ggf. in Tröpfchen einer Emulsion erreicht werden. Die Verknüpfung soll auf der Ebene von RNA und auch der genomischen DNA getestet werden.

LipH Cycle: Lichtgesteuerte Nukleinsäurereaktionen für die Point-of-Care Diagnostik

\begin{tabular}{l|l}
\hline $\begin{array}{l}\text { Projektleiter } \\
\text { Mittelgeber }\end{array}$ & Prof. Dr. sc. hum. Marcus Frohme \\
\hline Projektvolumen & $450.350 €$ \\
\hline Laufzeit & $01 / 2016-12 / 2018$ \\
\hline
\end{tabular}

In diesem Projekt soll eine neue, patentierbare und interdisziplinäre Technologie von Nukleinsäurereaktionen entwickelt werden. Diese arbeiten anders als klassische PCR statt mit Temperatur mit Lichtsteuerung. Sie können als Nachweissysteme für die Point-of-Care Diagnostik genutzt werden.

MALDI Blast - MALDI-TOF Massenspektrometrie Analytik zur Untersuchung von spreng- und explosivstoffbelasteten Liegenschaften und Gebäuden

\begin{tabular}{|c|c|}
\hline Projektleiter & Prof. Dr. sc. hum. Marcus Frohme \\
\hline Kooperationspartner & $\begin{array}{l}\text { terracon Laboratorium für Umwelt- und Pestizidanalytik GmbH, Prof. Dr. Machholz } \\
\text { Umweltprojekte GmbH }\end{array}$ \\
\hline Mittelgeber & Bund | BMWi | ZIM \\
\hline Projektvolumen & $193.072 €$ \\
\hline Laufzeit & $10 / 2014-03 / 2017$ \\
\hline \multicolumn{2}{|c|}{$\begin{array}{l}\text { Das Projekt hat die Etablierung einer innovativen Technik zum Nachweis von Spreng-/Explosivstoffverbindungen sowie } \\
\text { deren Abbauprodukten in Boden-/Grundwasserproben zum Ziel. Hierfür wird die MALDI-TOF-MS als Hochdurchsatz- } \\
\text { methode eingesetzt. Mit der zu entwickelnden Vor-Ort-Extraktion und -Probenvorbereitung werden die labilen Verbin- } \\
\text { dungen direkt am Probenentnahmeort konserviert. Zusätzlich wird der Einfluss der Bindung an Huminsäuren und die } \\
\text { Auswirkungen auf die Bioverfügbarkeit untersucht. }\end{array}$} \\
\hline
\end{tabular}

MALDI on-site-Prep - MALDI-Messmethodik zum Nachweis von Mikroorganismen in sprengstoffbelasteten Böden

Projektleiter

Prof. Dr. sc. hum. Marcus Frohme

Mittelgeber

Bund | BMWi | ZIM

Laufzeit

$10 / 2014-03 / 2017$

Entwicklung eines Hochdurchsatz-Nachweisverfahrens für Mikroorganismen in sprengstoffkontaminierten Böden. Mit Hilfe des MALDI-TOF Intact Protein Profiling werden die Zeigerorganismen für Sprengstoffkombinationen bestimmt. Zur schonenden Probengewinnung aus verschiedenen Bodenschichten wird ein neues Probenentnahmegerät (Multikapillarer-Mikroorganismen-Sampler) konstruiert. Zudem wird die Möglichkeit untersucht, intrazelluläre Sprengstoffverbindungen nachzuweisen. 


\section{MALDI-Coffee}

Projektleiter

Mittelgeber

Projektvolumen

Laufzeit
Prof. Dr. sc. hum. Marcus Frohme

Bund | BMWi | ZIM

$189.836 €$

03/2016-05/2018

Entscheidend für die Qualität von Röstkaffee ist die etwa einjährige Phase vor der Röstung. Ziel des Projekts ist die Charakterisierung dieser Reifungsprozesse. Dazu werden massenspektrometrische Analysemethoden entwickelt und mit sensorischen Geschmacksprofilen des Röstkaffees korreliert. Zur Optimierung der Reifungsprozesse wird eine Apparatur zur Begasung von Kaffeebohen konstruiert. Unterschiedliche Einflussfaktoren sollen untersucht und optimiert werden.

\section{MoMiSmart: Mobile Mikroskopie auf dem Smartphone}

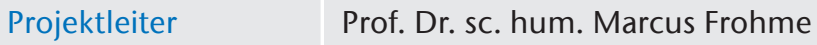

Mittelgeber Land Brandenburg I MWFK

Projektvolumen $\quad 84.000 €$

Laufzeit $\quad$ 03/2017-12/2018

MoMiSmart wird im Forschungscluster DigiLog (Digitale und analoge Begleiter für eine alternde Bevölkerung) im Rahmen des Gesundheitscampus durchgeführt. Das Projekt zielt auf die Entwicklung Smartphone-basierter Mikroskopielösungen für die Diagnostik. Im Fokus stehen hierbei Blutuntersuchungen. So könnte ein „Kleines Blutbild“ direkt am Bett des Patienten entstehen, wenn nicht auf eine Laboruntersuchung gewartet werden kann (bspw. bei akuten Infektionen).

SOCS2 CompDiag: Companion Diagnostics von Wachstumshormonstörungen basierend auf SOCS2- Komplexen

Projektleiter

Mittelgeber

Projektvolumen

Laufzeit
Prof. Dr. sc. hum. Marcus Frohme

Land Brandenburg | MWFK | EFRE | STaF

$397.000 €$

$03 / 2016-05 / 2018$

In diesem Projekt soll ein potenzieller Biomarker für Companion Diagnostics bei Wachstumshormondefizienz (GHD) oder Akromegalie validiert werden,d.h., eine Untersuchung von Veränderungen in der Gen- und Proteinexpression des SOCS2Komplexes. Im Anschluss soll dies als Lab-on-a-Chip-System implementiert werden durch Messung von zirkulierender zellfreier DNA im Blut.

\section{VBT-Ageing: Konsequenzen der altersassoziierten Zell- und Organfunktion}

Projektleiter

Kooperationspartner

Mittelgeber

Laufzeit
Prof. Dr. sc. hum. Marcus Frohme

\section{Wirtschaft}

Helmholtz-Zentrum Geesthacht

03/2017-12/2018

Untersuchung von Glycosilierungsvarianten des RSV-Proteins auf ihre Immunkompetenz für ältere Patienten. Ziel ist die Entwicklung von maßgeschneiderten Vakzinen für ältere Patienten. 
MolEpiArm: Molecular epidemiology and diagnostics of Leishmaniasis in Armenia

Projektleiter Dr. Katrin Kuhls

Kooperationspartner Alexsanyan Institute, Jerewan, Armenia; Trakia University, Stara Zagora, Bulgaria

Mittelgeber

DAAD

Projektvolumen $\quad 1.000 €$

Laufzeit

$10 / 2016-07 / 2017$

In the scientific focus are epidemiological screening studies on prevalence/distribution of Leishmaniasis in Armenia and its neighboring countries and sampling from infected humans and animals. The samples will be used for genotyping of the parasites. The results will help to understand the transmission cycles, the dynamics, origin and the way of spread of the disease including also neighboring countries and the prediction of risk factors. Training will be provided in molecular techniques.

Bioelektrokatalyse $2020+-$ Nutzung von Sonnenenergie für die Bioelektrokatalyse

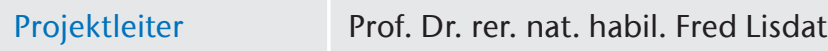

Mittelgeber Bund I BMBF

Projektvolumen $\quad 836.760 €$

Laufzeit $\quad 11 / 2012-10 / 2017$

Im Rahmen des Tandemvorhabens sollen lichtaktivierbare Elektroaufbauten für die biokatalytische Synthese entwickelt werden. Hierzu sollen zunächst Photosysteme oder Photosystemkomponenten mit Elektroden derart kombiniert werden, dass ein effektiver Photoelektronentransfer möglich wird. Durch die Kopplung unterschiedlicher Enzymsysteme mit photoaktivierbaren Elektroden soll dann der generierte Photostrom für die biokatalysierte Synthese wichtiger chemnischer Verbindungen genutzt werden.

CDN-Parkinson: Fluidisches System zur Detektion von bei der Parkinson-Erkrankung relevanten Enzymen und Medikamenten

\begin{tabular}{l|l} 
Projektleiter Prof. Dr. rer. nat. habil. Fred Lisdat & Prot
\end{tabular}

Mitarbeiter Dipl.-Biotechnol. Gero Göbel

Kooperationspartner BioTeZ Berlin-Buch GmbH, UP TRANSFER GmbH Potsdam

Mittelgeber $\quad$ Bund | BMWi | ZIM

\begin{tabular}{l|l} 
Projektvolumen $\quad 190.000 €$
\end{tabular}

Laufzeit $\quad 03 / 2016-08 / 2018$

Für eine individuelle Behandlung und Diagnose bei Parkinson-Patienten sollen im Rahmen einer personalisierten Medizin neue diagnostische Systeme entwickelt werden. Ziel des Kooperationsprojektes ist es Analyseverfahren zu entwickeln, die eine Bestimmung der Wirkeffizienz von Parkinsonmedikamenten ermöglichen und dadurch eine auf den Patienten maßgeschneiderte Therapie erlauben. 
EDS Detektor: Detektor zur energiedispersiven Röntgenspektroskopie EDS

\begin{tabular}{|c|c|}
\hline Projektleiter & Prof. Dr. rer. nat. habil. Fred Lisdat \\
\hline Mittelgeber & Land Brandenburg | MWFK | EFRE | InfraFEI \\
\hline Projektvolumen & $99.500 €$ \\
\hline Laufzeit & $11 / 2016-12 / 2017$ \\
\hline \multicolumn{2}{|c|}{$\begin{array}{l}\text { Bei der im Rahmen dieses Projektes beabsichtigten Beschaffung eines Detektors zur energiedispersiven Röntgenspektro- } \\
\text { skopie (EDS) sollen die bestehenden Fähigkeiten eines bereits etablierten Rasterelektronenmikroskops zur strukturellen } \\
\text { Charakterisierung von Oberflächen im Mirko- und Nanometerbereich um die Fähigkeit zur Elementanalyse erweitert wer- } \\
\text { den. Dies ist für zahlreiche Fragestellungen von Interesse, da es hier oft um die sinnvolle Kombination von verschiedenen } \\
\text { Materialien geht. }\end{array}$} \\
\hline
\end{tabular}

\section{NaWiTex - naturwissenschaftlich und technische Schülerlabore der TH Wildau}

\begin{tabular}{|c|c|}
\hline Projektleiter & Prof. Dr. rer. nat. habil. Fred Lisdat \\
\hline Mittelgeber & Land Brandenburg | MWFK | ESF \\
\hline Projektvolumen & $505.140 €$ \\
\hline Laufzeit & $06 / 2015-09 / 2017$ \\
\hline \multicolumn{2}{|c|}{$\begin{array}{l}\text { NaWiTex dient der Studienvorbereitung der TH Wildau und soll den Übergang an die Hochschule speziell in den MINT- } \\
\text { Studiengängen erleichtern. Es beinhaltet den Auf-/Ausbau von } 4 \text { Schülerlaboren im Fachbereich INW, die mit einer } \\
\text { einheitlichen Koordination und Außendarstellung versehen werden. Zielgruppen sind Schülerinnen und Schüler der } \\
\text { Sekundarstufe II (u.a. weibliche TN und TN aus bildungsfernen Haushalten). Des Weiteren soll das Schnupperstudium auf } \\
\text { Berufsqualifizierende erweitert werden. Ziel ist eine Teilnehmerzahl von } 4200 \text {. }\end{array}$} \\
\hline
\end{tabular}

\section{Proteinelektrochemie: Voltammetrische Charakterisierung von glycosyliertem Cytochrom c an SAMs}

\begin{tabular}{l|l}
$\begin{array}{l}\text { Projektleiter } \\
\text { Mittelgeber }\end{array}$ & Prof. Dr. rer. nat. habil. Fred Lisdat \\
\hline $\begin{array}{l}\text { Projektvolumen } \\
\text { DAAD }\end{array}$ & $10.000 €$ \\
\hline Laufzeit & $06 / 2017-11 / 2017$ \\
\hline $\begin{array}{l}\text { Im geplanten Projekt soll der Einfluss der Glycosylierung auf das elektrochemische Verhalten von globulärem Cytochrom c } \\
\text { untersucht werden, das sich frei in Lösung befindet oder eine Goldelektrode gebunden ist. Im Fokus der Forschung steht } \\
\text { dabei die Untersuchung der Auswirkungen der Glucosylierung auf den intrinsischen Ladungstransfermechanismus und } \\
\text { die heterogene Elektronentransferkonstante. }\end{array}$
\end{tabular}


STEM EU: Innovative Model of learning STEM in secondary schools

Projektleiter

Prof. Dr. rer. nat. habil. Fred Lisdat

Mittelgeber

EU | Erasmus+ | Key Action 2

Projektvolumen

$45.000 €$

Laufzeit

$11 / 2017-04 / 2020$

Das Projekt zielt auf die Stärkung der Fähigkeit von Sekundarschulen, Fähigkeiten in Fächern wie Wissenschaft, Technologie, Ingenieurwesen und Mathematik (STEM) durch innovative und interaktive pädagogische Methoden und Ansätze zu entwickeln. Es wird Schulen mit Werkzeugen und einem pädagogischen Modell ausstatten, das Schüler unterstützt, sich mit den „realen“ Anwendungen von STEM auseinanderzusetzen.

ForExChem: Forschendes Experimentieren in den modernen Chemiewissenschaften für Schüler

Projektleiter

Dr. Anke Renger

Kooperationspartner

Fond der Chemischen Industrie

Mittelgeber

Wirtschaft

Laufzeit

$10 / 2016-03 / 2018$

Mit dem vorliegenden Projekt sollen Schülern naturwissenschaftlich-technische Arbeitsmethoden vermittelt werden. Dies soll dazu führen, dass die Schüler ersten eigenen Ideen in den modernen Chemiewissenschaften nachgehen. Diese individuelle Förderung von MINT-interessierten Schülern und die Einbindung von Experimenten in das wissenschaftliche Arbeiten soll nach der Schule außerdem eine authentische Perspektive für einen Beruf oder ein Studium aufzeigen. Darüber hinaus gibt es Workshops für Lehrer. 


\section{TABELLARISCHE ÜBERSICHT PUBLIKATIONEN}

Akhoundi M, Downing T, Votýpka J, Kuhls K, Lukeš J, Cannet A, et al. (2017)

Amro A, Al-Dwibe H, Gashout A, Moskalenko O, Galafin M, Hamarsheh O, Frohme M, Jaeschke A, Schönian G, Kuhls K (2017)

Bay DH, Busch A, Lisdat F, lida K, Ikebukuro K, Nagasawa K, Karube I, Yoshida W (2017)

Ciornii D, Feifel SC, Hejazi M, Kölsch A, Lokstein H, Zouni A, Lisdat F (2017)

Ciornii D, Feifel SC, Hejazi M, Kölsch A, Lokstein H, Zouni A, Lisdat F (2017)

Fandrich A, Buller J, Memczak H, Stöcklein W, Hinrichs K, Wischerhoff E, Schulz B, Laschewsky A, Lisdat F (2017)

Fischbach J, Frohme M, Glökler JF (2017)

Fischbach J, Loh Q, Bier FF, Lim TS, Frohme M, Glökler JF (2017)

Fusco G, Göbel G, Zanoni R, Kornejew E, Favero G, Mazze F, Lisdat F (2017)

Gering V, Kober L, Brödel O, Koskei R, Mugendi B, Frohme M (2017)
Leishmania infections: Molecular targets and diagnosis Molecular Aspects of Medicine. 57, 1-29.

Spatiotemporal and molecular epidemiology of cutaneous leishmaniasis in Libya. PLoS Neglected Tropical Diseases 11(9):e0005873. doi: 10.1371/journal.pntd.0005873

Identification of G-quadruplex structures that possess transcriptional regulating functions in the Dele and Cdc6 CpG islands. BMC Molecular Biology 18:1-8. doi: 10.1186/s12867-017-0094-Z

Construction of photobiocathodes using multi-walled carbon nanotubes and photosystem I. Physica Status Solidi (A) - Applications and Materials Science 214(9):201700017. doi: 10.1002/pssa.201700017

Bioelectronic circuit on a 3D electrode architecture: Enzymatic catalysis interconnected with photosystem I. J Am Chem Soc 139(46):16478-16481. doi: 10.1021/jacs.7b10161

Responsive Polymer-Electrode Interface-Study of its Thermo- and $\mathrm{pH}$-Sensitivity and the Influence of Peptide Coupling. Electrochimica Acta 229:325-333. doi: 10.1016/j.electacta.2017.01.080

Hinge-initiated Primer-dependent Amplification of Nucleic Acids (HIP) - A New Versatile Isothermal Amplification Method. Scientific Reports 7:7683. doi: 10.1038/s41598-017-08067-x

Alizarin Red S for Online Pyrophosphate Detection Identified by a Rapid Screening Method. Scientific Reports 7:45085. doi: 10.1038/ srep45085

Polymer-supported electron transfer of PQQ-dependent glucose dehydrogenase at carbon nanotubes modified by electropolymerized polythiophene copolymers. Electrochimica Acta 248:64-74. doi: 10.1016/j.electacta.2017.07.105

LC-MS/MS als Methode zur Detektion und Quantifizierung der Inhaltsstoffe in Kaffee. Wissenschaftliche Beiträge 21:7-12. doi: 10.15771/0949-8214_2017_1 
Hornemann A, Sinning D, Cortes S, Campino L, Emmer P, Kuhls K, Ulm G, Frohme M, Beckhoff B (2017)

Kaltdorf KV, Schulze K, Helmprobst F, Kollmannsberger P, Dandekar T, Stigloher C (2017)

Karakus M, Nasereddin A, Onay H, Karaca E, Özkeklikci A, Jaffe CL, Kuhls K, Özbilgin A, Ertabaklar H, Demir S, Özbel Y, Töz S (2017)

Keil K, Dierenbach J, Schattschneider C, Enke H, Steuer R, Frohme M, Tillich UM (2017)

Kinuthia C, Frohme M, Brödel O, Mugendi B (2017)

Kovalenko DA, Kudratova Z, Moskalenko O, Lobstein S, Suvonkulov U, Strelkova M, Cortes S, Frohme M, Kuhls K (2017)

Lisdat F (2017)

Matuschek L, Göbel G, Lisdat F (2017)

Meier R, Graw S, Beyerlein P, Koestler D, Molina JR, Chien J (2017)

Moskalenko O, Sukiasyan A, Keshishyan A, Melik-Andreasyan G, Manukyan D, Atshemyan L, Frohme M, Cortes S, Kuhls K (2017)

Prylutska S, Grynyuk I, Grebinyk A, Hurmach V, Shatrava I, Sliva T, Amirkhanov V, Prylutskyy Y, Matyshevska O, Slobodyanik M, Frohme M, Ritter U (2017)

Radke L, Sandig G, Lubitz A, Schließer U, Horsten HH von, Blanchard V, Keil K, Sandig V, Giese C, Hummel M, Hinderlich S, Frohme M (2017)

Riedel M, Hölzel S, Hille P, Schörmann J, Eickhoff M, Lisdat F (2017)
A Pilot study on Fingerprinting Leishmania species from the Old World using Fourier transform infrared spectroscopy. Anal Bioanal Chem k.A: 1-17. doi: 10.1007/s00216-017-0655-5

FIJI Macro 3D ART VeSElecT: 3D Automated Reconstruction Tool for Vesicle Structures of Electron Tomograms. PLoS Computational Biology 13(1):e1005317. doi: 10.1371/journal.pcbi.1005317

Epidemiological Analysis of Leishmania tropica Strains and Slide Samples from Syrian and Turkish Leishmaniasis Patients using Multilocus Microsatellite Typing (MLMT). PLoS Neglected Tropical Diseases 11(4): 1-12. doi: 10.1371/journal.pntd.0005538

Photoscreener - ein integriertes Pipettierrobotersystem für Kultivierung und Screening von Mikroalgen in der Biotechnologie. In: Reiff-Stephan J (ed) Tagungsband AALE 2017. VDE VERLAG, Berlin-Offenbach, ISBN: 978-3-8007-4360-5

LCMS Analysis of Biochemical Composition in Different Kenyan Coffee Classifications. Food Science and Quality Management 69: 44-52

Leishmaniasis in Uzbekistan - an epidemiological update. 6th World Congress on Leishmaniasis, Toledo, Spain, p 1457

Trends in the layer-by-layer assembly of redox proteins and enzymes in bioelectrochemistry. Current Opinion in Electrochemistry 5(1):165-172. doi: 10.1016/j.coelec.2017.09.002

Electrochemical detection of serotonin in the presence of 5-hydroxyindoleacetic acid and ascorbic acid by use of 3D ITO electrodes. Electrochemistry Communications 81:145-149. doi: 10.1016/j.elecom.2017.07.003

digit - a tool for detection and identification of genomic interchromosomal translocations. Nucleic Acids Research 45(9): e72. doi: 10.1093/ nar/gkx010

Emerging foci of visceral leishmaniasis in Armenia - molecular epidemiology and pilot risk assessment by ecological niche modeling. 6th World Congress on Leishmaniasis, Toledo, Spain, p 254

Cytotoxic Effects of Dimorfolido-N-Trichloroacetylphosphorylamide and Dimorfolido-N-Benzoylphosphorylamide in Combination with C60 Fullerene on Leukemic Cells and Docking Study of Their Interaction with DNA. Nanoscale Research Letters 12(124): 1-9. doi: 10.1186/s11671-0171893-3

In Vitro Evaluation of Glycoengineered RSV-F in the Human Artificial Lymph Node Reactor. Bioengineering 4(3):70. doi: 10.3390/bioengineering4030070

InGaN/GaN nanowires as a new platform for photoelectrochemical sensors - detection of NADH. Biosensors and Bioelectronics 94:298-304. doi: 10.1016/j.bios.2017.03.022 
Riedel M, Lisdat F (2017)

Riedel M, Lisdat F (2017)

Riedel M, Sabir N, Scheller FW, Parak WJ, Lisdat F (2017)

Riedel M, Schäfer D, Lisdat F (2017)

Rogge C, Zinn S, Prosposito P, Francini R, Foitzik A (2017)

Sandig G, Horsten HH von, Radke L, Blanchard V, Frohme M, Giese C, Sandig V, Hinderlich S (2017)

Sukiasyan A, Keshishyan A, Manukyan D, MelikAndreasyan G, Atshemyan L, Frohme M, Cortes S, Kuhls K (2017)

Wettstein C, Kano K, Schäfer D, Wollenberger U, Lisdat F (2017)
Biosensorial Application of Impedance Spectroscopy with Focus on DNA Detection. In: Springer Series on Chemical Sensors and Biosensors. Springer, Berlin Heidelberg, ISBN: 2365-0729, pp 1-46. doi: 10.1007/5346_2017_18

Integration of enzymes in polyaniline-sensitized 3D inverse opal TiO2 architectures for light-driven biocatalysis and light-to-current conversion. ACS Applied Materials \& Interfaces. doi: 10.1021/acsami.7b15966

Connecting quantum dots with enzymes: Mediator-based approaches for the light-directed read-out of glucose and fructose oxidation. Nanoscale 9: 2814-2823. doi: 10.1039/C7NR00091]

Quantum Dot Architectures on Electrodes for Photoelectrochemical Analyte Detection. In: Grunwald P (ed) Biocatalysis and Nanotechnology. Pan Stanford, Singapore, ISBN: 9789814613699

Transmitted light pH optode for small sample volumes. Journal of Sensors and Sensor Systems 6(2):351 -359. doi: 10.5194/jsss-6-351-2017

Engineering of $\mathrm{CHO}$ Cells for the Production of Recombinant Glycoprotein Vaccines with Xylosylated N-glycans. Bioengineering 4(2): 1-12. doi: 10.3390/bioengineering4020038

Molecular Diagnosis of Visceral Leishmaniasis in Armenia. 6th World Congress on Leishmaniasis, Toledo, Spain, p 1389

Die Flavin-abhängige Fruktosedehydrogenase und Cytochrom c: Elektronentransfer und Sensorstrategien. Wissenschaftliche Beiträge 21: 13-21. doi: 10.15771/0949-8214_2017_2 


\section{FORSCHUNGSPREISE}

Prof. Dr. sc. hum. Marcus Frohme
Forschungsprofessur 2014-2019

der TH Wildau
„Molekulare Biotechnologie und Funktionelle Genomik“

\section{KOOPERATIVE PROMOTIONEN}

\begin{tabular}{|c|c|c|c|}
\hline Artur Fandrich & $\begin{array}{l}\text { Untersuchung des Verhaltens von ther- } \\
\text { moresponsiven Polymeren auf Elektro- } \\
\text { den in Interaktion mit biomolekularen } \\
\text { Systemen }\end{array}$ & Prof. Dr. habil. Fred Lisdat & Universität Potsdam \\
\hline Kai Stieger & $\begin{array}{l}\text { Nanobionic Strategies for the Imple- } \\
\text { mentation of Photosystem I into Biohyb- } \\
\text { rid Photoelectrodes }\end{array}$ & Prof. Dr. habil. Fred Lisdat & Humboldt Universität zu Berlin \\
\hline
\end{tabular}




\section{Informatik und Telematik Informatics and Telematics}




\section{SecAware4job: Informationssicherheitsbewusstsein für den Berufseinstieg}

Prof. Dr. rer. nat. Margit Scholl

PROJEKTMITARBEITER(INNEN) Frauke Fuhrmann, Denis Edich, Peter Koppatz (zeitweise), Peter Ehrlich (bis Ende 2016); Studentische Mitarbeiter: Benjamin Leiner, Robin Scholl

KOOPERATIONSPARTNER

PROJEKTVOLUMEN known_sense, Köln

MITTELGEBER

$199.805 €$

LAUFZEIT

Horst Görtz Stiftung

09/2015-08/2017

Die voranschreitende Digitalisierung aller Lebensbereiche erfordert ein höheres Bewusstsein für Informationssicherheit und entsprechende Kompetenzen zum Schutz sensibler Informationen - sowohl im Privat- als auch im Arbeitsleben. Insbesondere KMU können es sich oftmals nicht leisten, ihre Mitarbeiterinnen und Mitarbeiter in der umfassenden Weise für Informationssicherheit zu sensibilisieren und fortzubilden, die für ein langfristiges Informationssicherheitsbewusstsein und entsprechende Verhaltensweisen notwendig ist.

Vor diesem Hintergrund ist es das Ziel des Forschungsprojektes „SecAware4job: Informationssicherheitsbewusstsein für den Berufseinstieg“, Studierende als zukünftige Mitarbeiterinnen und Mitarbeiter für die alltäglichen Herausforderungen des Schutzes der Informationssicherheit und der digitalen Infrastruktur zu sensibilisieren sowie entsprechende Kompetenzen fundiert zu fördern. Denn nicht nur ITFachkräfte, sondern alle Mitarbeiterinnen und Mitarbeiter sollten über ein Informationssicherheitsbewusstsein und entsprechende Kompetenzen zum Schutz sensibler Informationen in ihrem Aufgaben- und Verantwortungsbereich verfügen.

Zur Erreichung dieses Ziels wird eine berufsorientierte Zusatzqualifikation konzipiert, die insbesondere in den nicht technischen Studiengängen als Wahlpflichtfach etabliert werden soll.
Um das abstrakte und komplexe Thema Informationssicherheit mit all seinen Facetten (z. B. rechtliche Rahmenbedingungen, Normen \& Standards, Schutzmaßnahmen) leicht verständlich sowie greif- und erlebbar zu vermitteln, wird eine Vielfalt an analogen (haptischen) und digitalen spielebasierten Lernszenarien (Serious Games) entwickelt und erprobt. Zum Nachweis ihrer erworbenen Kompetenzen im Hinblick auf Informationssicherheit haben die Studierenden der interaktiven Vorlesung die Möglichkeit, die Prüfung des Datenschutz- oder IT-SicherheitsModuls des Europäischen Computerführerscheins (ECDL) oder das Zertifikat zum IT-Sicherheitsbeauftragten (IT-SiBe), das fünf Jahre gültig ist, zu absolvieren. Während der Projektlaufzeit wird die Teilnahme an diesen Zertifikatsprüfungen durch Projektmittel gefördert und ist für die beteiligten Studierenden kostenfrei. Insgesamt haben im Rahmen der drei angebotenen Durchläufe der Vorlesung sieben Studierende und ein studentischer Mitarbeiter des Projektes die umfangreiche IT-SiBe-Prüfung erfolgreich bestanden. Drei weitere Studierende aus dem Wintersemester 16/17 sowie ein studentischer und zwei wissenschaftliche Mitarbeitende haben die ECDLPrüfung erfolgreich absolviert.

\section{KONTAKT}

margit.scholl@th-wildau.de

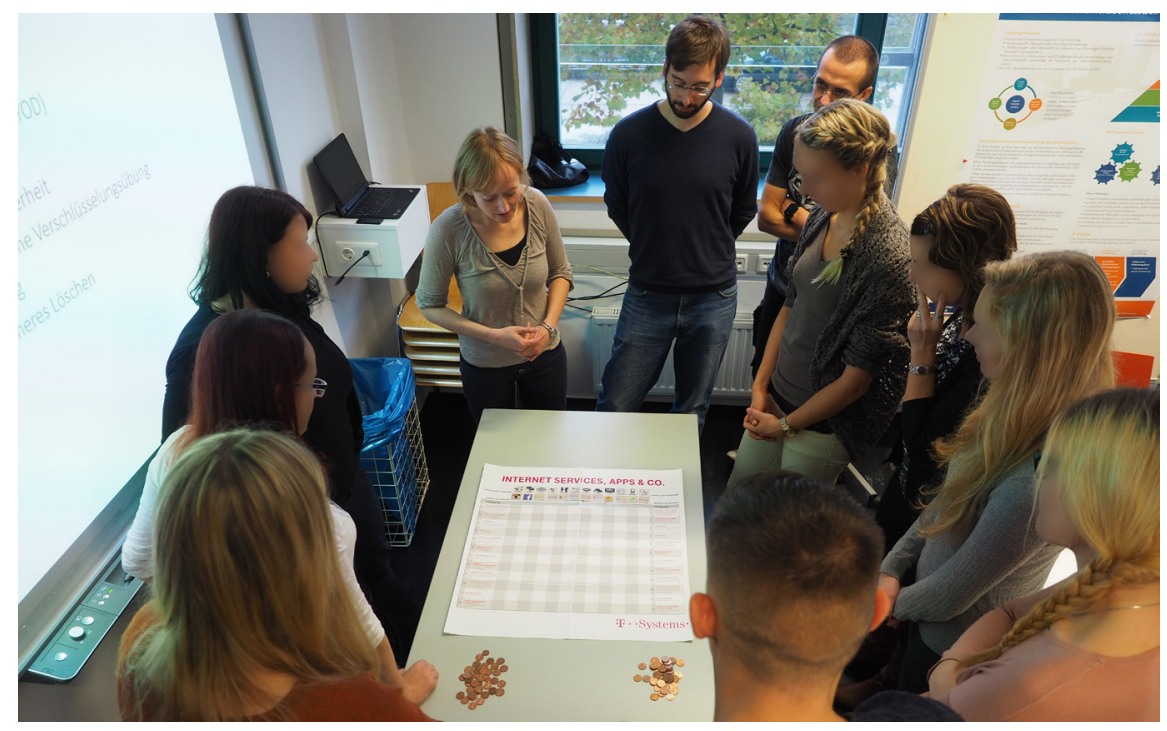

Abb. 1) Spielerische Sensibilisierung für die Verwendung von Apps und Internetdiensten 



\section{INNOMAP: Innenbereichs-Ortungssystem auf der Basis einer Bildanalyse markanter Punkte}

Prof. Dr. rer. nat. Ralf Vandenhouten

PROJEKTMITARBEITER(INNEN) Richard Fiebelkorn, Pascal Fechner

PROJEKTVOLUMEN

MITTELGEBER

LAUFZEIT
$334.340 €$

Land Brandenburg | MWFK | EFRE | StaF

01/2017-12/2019
Ortung und Navigation sind Verfahren, die seit Jahrhunderten verwendet und bereichsübergreifend eingesetzt werden. Nicht nur militärisch, sondern vor allem auch wirtschaftlich sind sie von essenzieller Bedeutung. Durch die ständige Weiterentwicklung existieren mittlerweile Technologien wie GNSS (u. a. GPS), die in unterschiedlichsten Prozessen Anwendung finden. Durch die Beschleunigung von Prozessen bei gleichzeitiger Qualitätssteigerung sind sie als wesentlicher Teil von Wertschöpfungsketten verschiedenster Branchen nicht mehr wegzudenken.

Allerdings sind bedeutsame Problemstellungen immer noch ungelöst. Nach wie vor gibt es kein Pendant zu GPS für den Indoor-Bereich, während alternative Ansätze, die auf Funkoder Infrarotortung basieren, die Anforderungen an Präzision, Zuverlässigkeit und Robustheit nicht erfüllen.
INNOMAP adressiert diese Problematik und fußt dabei auf einer Grundidee, für die die Technische Hochschule Wildau zum Schutz des geistigen Eigentums bereits ein Patent besitzt. Es verspricht, bei niedrigen Infrastrukturkosten eine Ortungs- und Navigationslösung zu realisieren, die sowohl außerhalb als auch innerhalb von Gebäuden eine Genauigkeit im Zentimeterbereich liefert.

Kernelement des Ortungsverfahrens in INNOMAP ist der Einsatz von Bildverarbeitung. Die Umgebung wird durch Kameras erfasst und im Anschluss von einem Computer verarbeitet. Dabei werden individuelle Landmarken erkannt und, mit einer zuvor eingespielten Datenbank abgeglichen, um dann mithilfe von mathematischen Ausgleichsverfahren die aktuelle Position der Kamera relativ zu den Landmarken zu berechnen. Die Landmarken können entweder zuvor erstellt und angebracht worden sein oder in Form

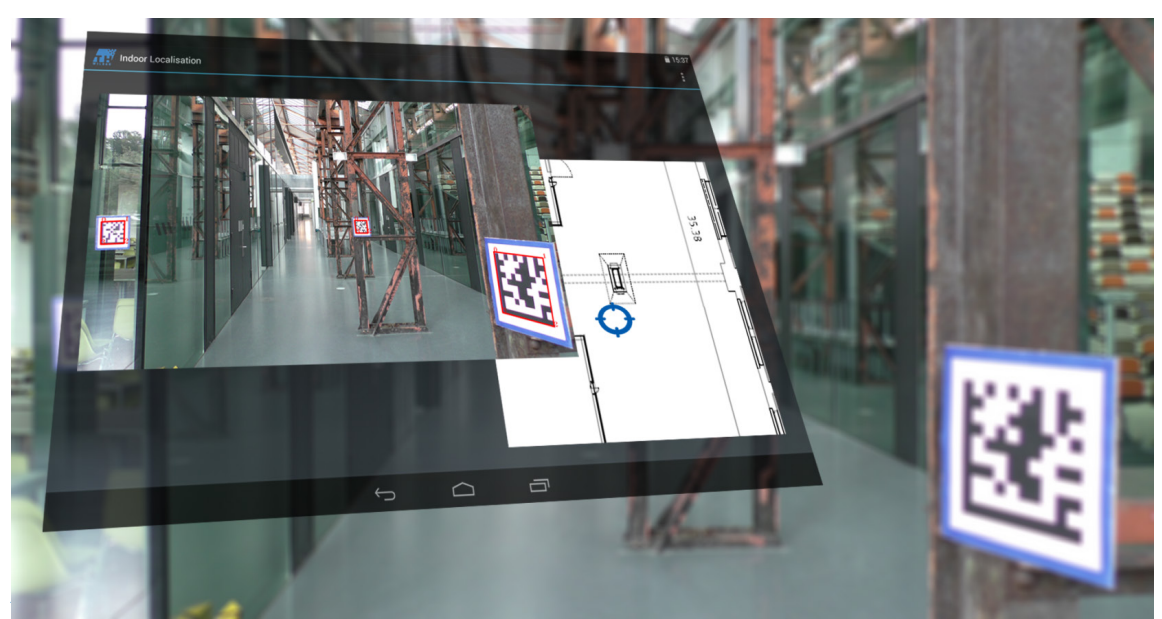

Grundsätzlich sind viele Anwendungen denkbar - von der Logistikbranche über den Handel bis zum Verkehr lässt sich mit präziser Ortung ein höherer Grad an Automation erreichen. Auch lassen sich Location-Based Services realisieren und in bereits vorhandene Infrastrukturen integrieren.

\section{PROJEKTWEBSEITE}

www.th-wildau.de/forschung-transfer/telematik/projekte/innomap 
Gefördert durch das Ministerium für Wissenschaft, Forschung und Kultur aus

Mitteln des Europäischen Fonds für regionale Entwicklung und des Landes Brandenburg.

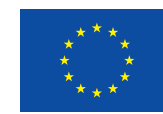

EUROPÄISCHE UNION

Europäischer Fonds für

Regionale Entwicklung

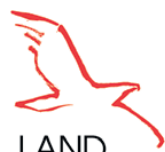

LAND

BRANDENBURG

Investition in Ihre Zukunft!

Ministerium für Wissenschaft,

Forschung und Kultur 


\title{
TABELLARISCHE ÜBERSICHT LAUFENDER PROJEKTE
}

\author{
ARES: Ablaufumgebung für einen Regelenergie- Leitstand \\ \begin{tabular}{l|l} 
Projektleiter Prof. Dr.-Ing. Michael Hendrix & Prix
\end{tabular} \\ Kooperationspartner Wirtschaft \\ Mittelgeber WWF Solar $\mathrm{GmbH}$ \\ Laufzeit $\quad 11 / 2016-08 / 2018$
}

Inhalt dieses Projektes ist die Konzeption und Entwicklung einer Hard- und Software als Ablaufumgebung für den operativen Betrieb eines Leitstandes zur Überwachung, Steuerung und Abrechnung eines Netzwerkes von Regelenergieanlagen.

\section{KIS-NI - Kampfmittelinformationssystem Niedersachsen}

Projektleiter

Prof. Dr.-Ing. Michael Hendrix

Mittelgeber

Landesamt für Geoinformation und Landvermessung Niedersachsen

Projektvolumen $409.735 €$

Laufzeit

12/2015-05/2020

Inhalt dieses Projektes ist die Erstellung einer Anwendungssoftware „Kampfmittelinformationssystem Niedersachsen (KIS-NI)“ zur digitalen Bearbeitung aller wesentlichen Geschäftsprozesse für den Kampfmittelbeseitigungsdienst Niedersachsen (KBD) in folgenden Leistungsstellen: Auftragsverwaltung, Luftbildauswertung, Räumstellendokumentation, Kampfmittelfund und -entsorgung, Berichtwesen, Datenhaltung in einer Kampfmitteldatenbank, Kampfmittelwebdienste

\section{KMBD- FIS 6. Modul: Kampfmittelbeseitigung Fachinformationssystem 6. Modul - 2016}

\begin{tabular}{l|l} 
Projektleiter $\quad$ Prof. Dr.-Ing. Michael Hendrix
\end{tabular}

Mittelgeber Land Brandenburg I Zentraldienst der Polizei

\begin{tabular}{l|l} 
Projektvolumen $\quad 125.300 €$
\end{tabular}

Laufzeit $\quad 11 / 2016-10 / 2017$

Die Bearbeitung des Projektes zur Implementierung des Fachinformationssystems für den Kampfmittelbeseitigungsdienst (KMBD-FIS) soll mit der Entwicklung eines sechsten Moduls zzgl. der Entwicklung einer App (KMBD-FIS Touch) und Durchführung eines Langzeitfeldversuches bezüglich des praktischen Einsatzes von KMBD-FIS Touch fortgesetzt werden. 
MI4G- Navigator: Bewertung der mobilen Datenverbindung

Projektleiter

Prof. Dr.-Ing. Michael Hendrix

Mittelgeber

Bund | BMWi | ZIM

Projektvolumen

$169.543 €$

Laufzeit

$09 / 2016-12 / 2017$

Inhalt des Projektes ist die Entwicklung einer Messmethode zur Nutzung semiprofessioneller Endgeräte (Smartphones) für die automatisierte Messung und Bewertung von Netz-, System- und Geräteperformance in Mobilfunknetzwerken mit dem Ziel der Ermittlung von Leistungsindizes und leichtverständlicher Darstellung der Reports in Form nutzerspezifischer Interpretationsmatrizen unter Berücksichtigung der relevanten Einflüsse.

SensoSphere: Mobiler sensorbasierter Kugelroboter zur spontanen Aufklärung sicherheitskritischer Situationen

\begin{tabular}{l|l} 
Projektleiter $\quad$ Prof. Dr.-Ing. Michael Hendrix
\end{tabular}

Mittelgeber $\quad$ Bund | BMWi | ZIM

Projektvolumen $\quad 190.000 €$

Laufzeit $\quad 08 / 2016-07 / 2018$

Ziel dieses Projektes ist die Entwicklung eines mobilen, fernsteuerbaren, mit Kameras und Sensoren ausgestatteten KugelRoboters, der mittels Wurf oder Luftdruck-Schussgerät in ein Beobachtungsgebiet verbracht werden kann, ohne dass ein Operator in dieses Gerät eindringen kann. SensoSphere soll als jederzeit einsetzbares und einfach zu handhabendes System zur spontanen Feldaufklärung bei Großschadensereignissen und Gefährungslagen sowohl indoor als auch outdoor verwendbar sein. Die Sensorik und die Kommunikationsmöglichkeiten des Systems sind modular konzipiert und lassen sich damit spontan an die Erfordernisse des jeweiligen Einsatzszenarios vor Ort anpassen.

AM@Ferrari: Optimierung des Anforderungsmanagements in der Elektronik- Entwicklung bei Ferrari S. p. A.

Projektleiter Prof. Dr.-Ing. Stefan Kubica

Mittelgeber Ferrari S.p.A.

Laufzeit $\quad 12 / 2015-02 / 2017$

Ziel dieses Projektes ist es, die Digitalisierung im Bereich des Anforderungsmanagements elektrischer/elektronischer Fahrzeugfunktionen bei Ferrari S.p.A. zu steigern. Hierbei geht es u.a. um die Einführung entwicklungsbegleitender Kennzahlen, datenbankorientiertem Anforderungsmanagement und dazugehörigen Prozessbeschreibungen. 
RFID@Crashsensorik_VW: Initialisierung einer Machbarkeitsanalyse zur Auslegung von Crashsensoren auf RFID- Basis

Projektleiter

Prof. Dr.-Ing. Stefan Kubica

Kooperationspartner Wirtschaft

Mittelgeber Volkswagen AG

Laufzeit $\quad 10 / 2016-02 / 2017$

Durch stetig steigende Anforderungen und komplexer werdende Unfallszenarien wird permanent nach neuen Sensortechnologien zur Unfallsensierung in punkto Leistungsvermögen und Kosten gesucht. Neben den bekannten Technologien (Druck- und Beschleunigungsmessung) haben sich in anderen Bereichen diverse Technologien etabliert. Das vorliegende Projekt untersucht die Potentiale von RFID-Technologie für den Einsatz in der Unfallsensierung.

\section{SecAware4job - Informationssicherheitsbewusstsein für den Berufseinstieg}

Projektleiter

Mitarbeiter

Kooperationspartner

Mittelgeber

Projektvolumen

Laufzeit

Projekthomepage
Prof. Dr. rer. nat. Margit Scholl

Frauke Fuhrmann, Denis Edich, Peter Ehrlich, Peter Koppatz

known_sense

Horst Görtz Stiftung

$199.805 €$

$09 / 2015-08 / 2017$

www.secaware4job.wildau.biz

SecAware4job verfolgt das Ziel, Studierende nicht-technischer Studiengänge als zukünftige Mitarbeiter/innen für die alltäglichen Herausforderungen des Schutzes der Informationssicherheit und der digitalen Infrastruktur zu sensibilisieren. Zur Erreichung dieses Ziels wird eine berufsorientierte Zusatzqualifikation für Studierende in Form einer innovativen Weiterbildung zur Stärkung des Bewusstseins und der Kompetenzen bzgl. Informations- und insbesondere IT-Sicherheit entwickelt. Diese Zusatzqualifikation soll zielgruppenspezifisch aufgebaut und vor allem in den nicht-technischen Studiengängen der TH Wildau direkt integriert werden. Die Studierenden erhalten die Möglichkeit, ein vierstufiges Zertifikat zu erwerben: vom einfachen Teilnahme- bzw. Moderationszertifikat, über den Europäischen Computerführerschein (ECDL) in den Modulen IT-Sicherheit und/oder Datenschutz, bis hin zum fünf Jahre gültigen Zertifikat IT-Sicherheitsbeauftragte/r (IT-SiBe). Um das abstrakte und komplexe Thema Informationssicherheit mit all seinen Facetten (z. B. rechtliche Rahmenbedingungen, Normen \& Standards, Schutzmaßnahmen, Konzepte) verständlich sowie greif- und erlebbar zu vermitteln, werden kreative Lehr- und Lernmethoden, u. a. analoge und digitale spielebasierte Lernszenarien, entwickelt und erprobt. 
Projektleiter

Mitarbeiter

Mittelgeber

Projektvolumen

Laufzeit

Projekthomepage

Prof. Dr. rer. nat. Margit Scholl

Frauke Fuhrmann, Denis Edich

Bund | BMBF

$240.832 €$

09/2017-08/2019

www.security.wildau.biz

Das Ziel des vom BMBF geförderten Projektes „Security“ liegt in der Weckung des Interesses von jungen Frauen (Schülerinnen) für den Beruf der Security Spezialistin. Dadurch sollen Studiengänge und Ausbildungen mit Informatikbezug für junge Frauen attraktiver werden und der Frauenanteil in diesen erhöht werden. Durch eine ansprechende und gendersensible Darstellung des Berufsbildes Security Spezialistin, durch Porträts von weiblichen Rollenvorbildern, die im Bereich Informationssicherheit tätig sind, sowie durch eine interaktive und erlebnisorientierte Pilotmaßnahme können Schülerinnen erfahren, dass Studiengänge und Ausbildungen mit Informatikbezug nicht nur technisch, sondern sehr vielseitig sind. Abgerundet wird das Projekt durch Informationsveranstaltungen, in denen sich Eltern, Lehrkräfte, MINT-Initiativen, Berufsberatungen, IHK etc. über das entwickelte Berufsbild und die Pilotmaßnahme sowie die Ausleihmöglichkeiten der entwickelten Materialien (z. B. spielebasierte Lernszenarien) nach Projektende informieren können.

\section{Skill Up: Matching graduates'skills and labour world demands through authentic learning scenarios}

Projektleiter

Mitarbeiter

Kooperationspartner

Mittelgeber

Projektvolumen

Laufzeit

Projekthomepage
Prof. Dr. rer. nat. Margit Scholl

\section{Frauke Fuhrmann}

Fundacio Per A La Universitat Oberta De Catalunya (Uoc) (Federführung), Institut Obert De

Catalunya (loc), Stockholms Universitet (Su), Hochschule der Bundesagentur für Arbeit (HdBA), Fundació Factor Humá

EU | Erasmus+ | KA2 Strategic Partnerships

$17.881 €$

$09 / 2016-02 / 2019$

Skill Up answers the urgent priority established by Europe 2020 policies of promoting graduates' employability by means of strengthen ties between higher education (HE) and VET curricula and the labour world through authentic learning scenarios and career counselling and guidance. The project sets the stage for scaling up innovative learning approaches, ICT-based methodologies, greater inter-sector cooperation, and the European-wide transfer of a replicable process that responds to real world work demands.A strong transnational strategic cooperation between four universities with diverse backgrounds (online and face-to-face settings, as well applied, technical and research oriented), an online VET (vocational and educational training) institution, and an employer representative from three European countries (Spain, Sweden and Germany) is built to carry out this project. 
Projektleiter

Mittelgeber

Projektvolumen

Laufzeit

Projekthomepage
TechPedia: European Virtual Learning Platform for Electrical and Information Engineering

EU | Erasmus+

$28.543 €$

09/2014-08/2017

www.techpedia.eu

Im Projekt erstellen jeweils eine Hochschule und ein Ausbildungsbetrieb in jeweils fünf Partnerländern Lernmaterialien für den technischen \& IT-Bereich. Alle Lernmaterialien werden auf einem Bildungsportal und für Lernplattformen (z.B. Moodle), als PDFs, für eBook-Reader und Smartphones zur Verfügung stehen. Auf internationaler Ebene soll zudem ein großer Wettbewerb durchgeführt werden, die Technische Olympiade.

BERMUDA - Bilderkennung multiperspektivischer Daten zur intelligenten Analyse von Gesten und Aktionsmustern

Projektleiter

Prof. Dr. rer. nat. Ralf Vandenhouten

Mittelgeber

Bund | BMBF | FHprofUnt

Projektvolumen

Laufzeit

$01 / 2015-12 / 2017$

Ein Bedarfsfeld der Hightech-Strategie 2020 ist die Sicherheit der Bürgerinnen und Bürger der Gesellschaft. Zum Bedarfsfeld der Sicherheit zählt u.A. der Schutz der Gesellschaft vor Terrorismus und Kriminalität. Dieses Forschungsprojekt widmet sich der Fragestellung des Einsatzes von stereo- und multiperspektiven Kameras zur Erkennung von Delikten des öffentlichen Personennahverkehrs (ÖPNV)sowie auf öffentlichen Plätzen (z.B. Bahnhöfe, Flughäfen).

\section{ENDOKMA: Endoskopische Orientierung in komplexen Maschinengeometrien}

Projektleiter

Prof. Dr. rer. nat. Ralf Vandenhouten, Prof. Dr. rer. nat. habil. Sigurd Schrader

Mittelgeber

Land Brandenburg | MWFK | EFRE | StaF

Projektvolumen $468.686 €$

Laufzeit

01/2017-09/2020

Endokma beschäftigt sich mit der anwendungsnahen Forschung eines innovativen Messsystems zur Orientierung in komplexen Maschinengeometrien. Ziel ist es, damit einen automatisierten Prozess bereitzustellen, der den Inspektions- bzw. Fertigungsprozess vereinfacht und optimiert. Die angestrebte Lösung verbindet dabei ein technisches Edoskop mit einer Kamera sowie verschiedenen MEMS (Micro Electro-Mechanical Systems) und Multisensordatenfusion. 
INNOMAP: Innenbereichs- Ortungssystem basierend auf der Bildanalyse markanter Punkte

Projektleiter

Prof. Dr. rer. nat. Ralf Vandenhouten

Mittelgeber Land Brandenburg | MWFK | EFRE | StaF

Projektvolumen $334.340 €$

Laufzeit

03/2016-11/2019

INNOMAP beschäftigt sich mit der anwendungsnahen Forschung eines innovativen Verfahrens zur Ortung und Navigation. Die angestrebte Lösung lässt eine bisher unerreichte Präzision erwarten und kann - im Gegensatz zu existierenden Verfahren - insbesondere auch im Innenbereich angewendet werden. Die einfache, ressourcenschonende sowie kostengünstige Anwendbarkeit des innovativen Konzeptes kann existierende Prozesse verbessern und neue Anwendungsgebiete erschließen. 


\section{TABELLARISCHE ÜBERSICHT PUBLIKATIONEN}

Barton T, Müller C, Seel C (2017)

Brüggemann R, Koppatz P, Fuhrmann F, Scholl M $(2017$

Eylert B (2017)

Fuhrmann F, Koppatz P, Edich D, Scholl M (2017)

Fuhrmann F, Scholl M, Brüggemann R (2017)

Fuhrmann F, Scholl M, Edich D, Koppatz P, Scholl LR, Leiner KB, Ehrlich P (2017)

Gedat E, Fechner P, Fiebelkorn R, Vandenhouten R (2017)

Jacob T, Kubica S (2017)

Koppatz P, Bruggemann R (2017)

Kubica S, Ringshausen H, Reiff-Stephan J, Schlingelhof M (2017)

Medaglia R, Loukis EN, Scholl M (2017)
Geschäftsprozesse. Von der Modellierung zur Implementierung. In: Angewandte Wirtschaftsinformatik. Springer Fachmedien Wiesbaden, Wiesbaden, ISBN: 978-3-658-17297-8. doi: 10.1007/9783-658-17297-8

A Matching Problem, Partial Order, and an Analysis Applying the Copeland Index. In: Bruggemann MF\&R (ed) Partial Order Concepts in Applied Sciences. Springer International Publishing, Cham, ISBN: 978-3319-45421-4, pp 231-238. doi: 10.1007/978-3-319-45421-4_16

Gott denken - für einen Naturwissenschaftler denkbar? Wissenschaftliche Beiträge 21:37-44. doi: 10.15771/0949-8214_2017_5

Sicher unterwegs in der digitalen Welt - spielend begreifen. VM Verwaltung \& Management 23(5):263-266. doi: 10.5771/0947-98562017-5-263

How Can the Empowerment of Employees with Intellectual Disabilities Be Supported? Social Indicators Research 133:1-17. doi: 10.1007/ s11205-017-1666-5

Informationssicherheitsbewusstsein für den Berufseinstieg. Shaker, Aachen. ISBN: 978-3-8440-5466-8. doi: 10.2370/9783844054668

Szenenanalyse und Unterscheidung der Skelette mehrerer Menschen in digitalen Bildern mit Graphentheorie durch eine k-kürzestedisjunkte-Wege-Suche. Wissenschaftliche Beiträge 21:31-35. doi: 10.15771/0949-8214_2017_4

Einsatz von Stream-Machine-Learning auf Fahrzeugdaten. In: Kubica S, Ringshausen H, Reiff-Stephan J, Schlingelhof M (eds) 2. Automobil Symposium Wildau Tagungsband Technische Hochschule Wildau 2017. Automobil Symposium Wildau. Technische Hochschule Wildau, Wildau, pp 39-46. doi: 10.15771/ASW_2017_6

„PyHasse“ and Cloud Computing. In: Bruggemann MF\&R (ed) Partial Order Concepts in Applied Sciences. Springer International Publishing, Cham, ISBN: 978-3-319-45421-4, pp 291-300. doi: 10.1007/978-3-31945421-4_20

2. Automobil Symposium Wildau. Tagungsband Technische Hochschule Wildau 2017. Automobil Symposium Wildau. Technische Hochschule Wildau, Wildau

Social Media and Government Minitrack (Introduction). In: Proceedings of the 50th Annual, ISBN: 978-0-9981331-0-2 
Müller C, Laue R (2017)

Ruiz Ben E, Scholl M, Ehrlich P (2017)

Scholl M (2017)

Scholl M (2017)

Scholl M (2017)

Scholl M, Fuhrmann F (2017)

Scholl M, Fuhrmann F, Edich D, Ehrlich P, Leiner B, Scholl R, Koppatz P (2017)

Scholl M, Leiner KB, Fuhrmann F (2017)

Scholl M, Leiner KB, Fuhrmann F (2017)

Tolkiehn G-U, Lührs C, Weigert PM (2017)

Vandenhouten R, Handrich J, Wendlandt O (2017)

Vandenhouten R, Hermerschmidt A, Fiebelkorn R (2017)
Simulation von Geschäftsprozessen: Werkzeuge, Standards und Fallstricke. In: Barton T, Müller C, Seel C (eds) Geschäftsprozesse: Von der Modellierung zur Implementierung. Angewandte Wirtschaftsinformatik. Springer Fachmedien Wiesbaden, Wiesbaden, ISBN: 978-3-658-17297-8, pp 25-43. doi: 10.1007/978-3-658-17297-8_3

Exploring the Adoption of E-Government Tools with WISE Work Index. Wissenschaftliche Beiträge 21:45-53. doi: 10.15771/0949-8214_2017_6

Forschendes Lernen aus der Perspektive der Fachhochschulen. In: Mieg HA, Lehmann J (eds) Forschendes Lernen: Wie die Lehre in Universität und Fachhochschule erneuert werden kann. Campus, Frankfurt, ISBN: 978-3-5935-0140-6, pp 401-409

IT-Security Awareness in the Field of Urban and Regional Planning. In: Lisbon, 28 slides. doi: 10.13140/RG.2.2.31594.72645

IT-Security Awareness in the Field of Urban and Regional Planning. In: II Conference Urban E-Planning, Lisbon, 28 slides. doi: 10.13140/ RG.2.2.31594.72645

Living in a Digital World: Improving Skills to Meet the Challenges of Digital Transformation Through Authentic and Game-Based Learning. Invited Keynote paper. Journal of Systemics, Informatics and Cybernetics 15(6):6

Das Projekt SecAware4job: Auf spielerischem Weg zu erhöhtem Informationssicherheitsbewusstsein für den Berufseinstieg. Wissenschaftliche Beiträge 21:23-30. doi: 10.15771/0949-8214_2017_3

Blind Spot: Do You Know the Effectiveness of Your Information Security Awareness-Raising Program? Journal of Systemics, Informatics and Cybernetics 15(4):58-62

Blind spot: Do you know the effectiveness of your information security awareness-raising program? In: Proceedings Vol. I. International Institute of Informatics and Systemics (IIIS), Orlando, Florida, USA, pp 361-366

ÖPNV 4.0. In: Kubica S, Ringshausen H, Reiff-Stephan J, Schlingelhof M (eds) 2. Automobil Symposium Wildau. Tagungsband Technische Hochschule Wildau 2017. Automobil Symposium Wildau. Technische Hochschule Wildau, Wildau, pp 3-10. doi: 10.15771/ASW_2017_1

Bildverarbeitung und Augmented Reality zur Erfassung realweltlicher Kontextinformationen in Mensch-Maschine-Schnittsteilen bei der Unterstützung von digitalisierten Arbeitsprozessen. In: Reiff-Stephan J (ed) Tagungsband AALE 2017. VDE VERLAG, Berlin•Offenbach, ISBN: 978-38007-4360-5, pp 77-88

Design and quality metrics of point patterns for coded structured light illumination with diffractive optical elements in optical 3D sensors. Proceedings of SPIE 10335:1033518. doi: 10.1117/12.2270248 


\section{Optische Technologien und Photonik Optical Technologies and Photonics}




\section{TABELLARISCHE ÜBERSICHT LAUFENDER PROJEKTE}

\section{DiaS: Erweiterung eines Beschichtungssystems zur Herstellung diamantartiger Schichten}

\begin{tabular}{|c|c|}
\hline Projektleiter & Prof. Dr. rer. nat. habil. Sigurd Schrader \\
\hline Mittelgeber & Land Brandenburg | MWFK | EFRE | InfraFEI \\
\hline Projektvolumen & $74.527 €$ \\
\hline Laufzeit & $09 / 2016-06 / 2018$ \\
\hline \multicolumn{2}{|c|}{$\begin{array}{l}\text { Im vorliegenden Vorhaben soll ein vorhandenes Plasmabeschichtungssystem technisch erweitert werden. Die Erweite- } \\
\text { rung dient der Herstellung diamantähnlicher Kohlenstoffschichten für den Schutz und zur gezielten Funktionalisierung } \\
\text { von Oberflächen hauptsächlich im Bereich der Sensorik. Damit sollen Oberflächen einerseits gegenüber äußeren Umwelt- } \\
\text { einflüssen wie zum Beispiel Feuchtigkeit und chemischen Angriffen unempfindlich werden. }\end{array}$} \\
\hline
\end{tabular}

Entwicklung eines neuen Gitters und Mitarbeit bei der Montage eines neuen Endoskops für das Forschungsthema „Entwicklung eines endoskopischen Messkopfes mit Schrägsicht-Optik“
Projektleiter
Prof. Dr. rer. nat. habil. Sigurd Schrader

\section{Mitarbeiter}
Dr. Joachim Max Bauer
Kooperationspartner Wirtschaft
Mittelgeber
Rolls-Royce Deutschland Ltd \& Co KG
Laufzeit
01/2017-02/2017

Zielsetzung ist die Detektion und Vermessung kleinster Oberflächenschädigungen im Mikrometerbereich mit hoher Sicherheit durch ein verbessertes Gitter für die Gitterprojektion.

EOFP: Entwicklung elektro-optisch abstimmbarer Fabry-Perot Ultraschallsensoren für biomedizinische photoakustische Bildgebung mit hoher Bildrate

\begin{tabular}{l|l}
$\begin{array}{l}\text { Projektleiter } \\
\text { Mittelgeber }\end{array}$ & Prof. Dr. rer. nat. habil. Sigurd Schrader \\
\hline $\begin{array}{l}\text { Projektvolumen } \\
\text { DFG }\end{array}$ & $211.300 €$ \\
\hline $\begin{array}{l}\text { Laufzeit } \\
\text { 03/2016-02/2019 }\end{array}$ \\
\hline $\begin{array}{l}\text { Ziel des interdisziplinären Projektes ist die Entwicklung elektro-optisch abstimmbarer Fabry-Perot-Ultraschallsensoren für } \\
\text { biomedizinische photoakustische Bildgebung mit hohen Bildraten. Das Projekt wird in Kooperation mit der TU Berlin } \\
\text { durchgeführt. Es umfasst Entwicklungsarbeiten in den Bereichen der nichtlinearen Optik, Polymerchemie, optischen Be- } \\
\text { schichtungstechnologie, optischen Ultraschallmessung und photoakustischen Bildgebung. }\end{array}$
\end{tabular}


EVD: Schichtbildung durch elektronenaktivierte Vakuum-Deposition

Projektleiter

Prof. Dr. rer. nat. habil. Sigurd Schrader

Mittelgeber

Bund | BMBF

Projektvolumen

$703.574 €$

Laufzeit

$04 / 2016-03 / 2018$

Ziel dieses Vorhabens ist es, Forschungsergebnisse der TH Wildau auf dem Gebiet der Vakuum-Gasphasen-Abscheidung kohlenstoffbasierter Funktionsmaterialien als Grundlage neuer Beschichtungstechnologien zu nutzen. Der Projektverbund strebt die Etablierung einer regionalen Entwicklungs- und Technologieplattform an, welche neue Polymer- bzw. Kohlenstoffbeschichtungsverfahren wie den EVD-Prozess und damit herstellbare Funktionsschichten ins Zentrum der gemeinsamen F\&E-Aktivitäten stellt.

FibrOLED: Faserförmige Organische Leutdioden

Projektleiter

Prof. Dr. rer. nat. habil. Sigurd Schrader

Mittelgeber

OSRAM GmbH

Laufzeit

$12 / 2016-03 / 2017$

Das Projekt dient der Entwicklung neuartiger organischer Leuchtdioden auf nichtplanaren Substraten und eines neuartigen Herstellungsverfahrens, welches Chemisorptionsprozesse aus der Flüssigphase nutzt.

\section{FuZiOn: Funktionelle Gestaltung metallischer Zifferblätter durch Laser induzierte Oxidation}

Projektleiter

Mitarbeiter

Mittelgeber

Projektvolumen

Laufzeit
Prof. Dr. rer. nat. habil. Sigurd Schrader

\section{Torsten Döhler}

Deutsche Bundesstiftung Umwelt

$60.000 €$

$01 / 2017-12 / 2017$

Ziel des Projekts ist die Entwicklung eines temperaturunterstützen laserbasierten, umweltfreundlichen Verfahrens zur Generierung langzeitstabiler, farblich in weiten Grenzen variierbarer Beschichtungen von Uhrenzifferblättern auf Titan. Durch das laserbasierte Verfahren zur Oberflächenmodifikation unter Anwendung einer selektiven Oberflächenoxidation sollen die Probleme, handwerklichen Schwierigkeiten und technischen Barrieren der zurzeit genutzten Beschichtungsverfahren reduziert werden. 
Graphen: Graphen-Netzwerk

\begin{tabular}{|c|c|}
\hline Projektleiter & Prof. Dr. rer. nat. habil. Sigurd Schrader \\
\hline Mitarbeiter & Dr. Friedhelm Heinrich \\
\hline Kooperationspartner & $\begin{array}{l}\text { FAP, Sentech, MRT, OEG, LaMont, Canlas, Golares, Sawall, } 5 \text { micron, IHP, Paul Drude Institut, } \\
\text { AMO GmbH, OUT }\end{array}$ \\
\hline Mittelgeber & Bund | BMWi | ZIM Netzwerk \\
\hline Projektvolumen & $171.398 €$ \\
\hline Laufzeit & $08 / 2017-07 / 2018$ \\
\hline \multicolumn{2}{|c|}{$\begin{array}{l}\text { Projektgegenstand ist die Gründung und der Aufbau eines ZIM-Netzwerkes "Graphen". Das Netzwerk soll Innovationen } \\
\text { auf dem Gebiet der kohlenstoffbasierten Funktionsschichten mit Blick auf die Erfordernisse der regionalen Wirtschaft ent- } \\
\text { wickeln und deren Verwertung unterstützen. Der Schwerpunkt liegt dabei auf dem Gebiet der Graphenherstellung, der } \\
\text { Graphenanalytik und der Anwendung von Graphenschichten. }\end{array}$} \\
\hline
\end{tabular}

\section{HOPBIT: Hybride Silizium-Organik-Photonik für die hochbitratige Datenübertragung}

\begin{tabular}{|c|c|}
\hline Projektleiter & Prof. Dr. rer. nat. habil. Sigurd Schrader \\
\hline Mittelgeber & Land Brandenburg | MWFK | EFRE | StaF \\
\hline Projektvolumen & $273.747 €$ \\
\hline Laufzeit & $01 / 2017-09 / 2020$ \\
\hline \multicolumn{2}{|c|}{$\begin{array}{l}\text { Das geplante Vorhaben basiert auf einer interdisziplinären Zusammenarbeit zwischen dem Leibniz-Institut für innovative } \\
\text { Mikroelektronik IHP und der Arbeitsgruppe für Photonik, Laser- und Plasmatechnologien der Technischen Hochschule } \\
\text { Wildau. Ziel des Vorhabens ist die Entwicklung einer Technologieplattform für die Integration von photonischen Silizium- } \\
\text { Organik-Hybrid (SOH) Bauelementen in eine siliziumbasierte Chiptechnologie. Die SOH Photonik ermöglicht eine hoch- } \\
\text { bitratige Datenübertragung. }\end{array}$} \\
\hline
\end{tabular}

\section{HOLMES: Systematische Untersuchung des nicht ordnungsgemäßen Betriebs von PIDs}

Projektleiter

Mittelgeber

Laufzeit
Prof. Dr. rer. nat. habil. Sigurd Schrader

Analytic Control Instruments $\mathrm{GmbH}$

08/2016-05/2017

Ziel des vorliegenden Projektes ist es, durch systematische Untersuchungen des nicht ordnungsgemäßen Betriebs von PIDs für verschiedene Umweltbedingungen im Hinblick auf die Abhängigkeit des Messsignals von der Transmission des Lampenfensters Schwachstellen und Ursachen im Aufbau der PIDs zu lokalisieren und durch geeignete Maßnahmen die Problematiken zu beheben. 
MultiFlexjoin: Verfahren zum Fügen komplexer Bauteile auf Basis direkt abgeschiedener reaktiver

Multischichtsysteme Herstellung direkt abgeschiedener Multischichtsysteme mittels PVD-Verfahren

Projektleiter

Prof. Dr. rer. nat. habil. Sigurd Schrader

Mitarbeiter

Claus Villringer

Kooperationspartner

MATRIX-GmbH, SenVac Anlagenbau Komponenten GmbH, TU Berlin, Institut für Werkzeugmaschinen und Fabrikbetrieb (IWF) - PTZ 1

Mittelgeber

Bund | BMWi | ZIM Kooperationsprojekt

Projektvolumen

$157.591 €$

Laufzeit

$10 / 2017-09 / 2020$

Nanoskalige reaktive Miltischichtsysteme (RMS) bieten die Möglichkeit Fügeprozesse mit einem definierten Wärmeeintrag zu initiieren. Der innovative Kern des Projekts besteht im Fügen von temperaturkritischen Werkstücken mit komplexen Freiformoberflächen mit für die Mikrotechnik charakteristischen Bauteilfeatures basierend auf direkt abgeschiedenen RMS sowie eines automatisierten Fügeprozesses durch intelligente und flexible Spann- und Aufnahmevorrichtungen.

Speculum 3: Chrom- und Goldtestbeschichtung von Glassubstraten

Projektleiter

Prof. Dr. rer. nat. habil. Sigurd Schrader

Mitarbeiter

Mandy Hofmann

Kooperationspartner Wirtschaft

Mittelgeber

SENTECH Instruments GmbH

Laufzeit

09/2017

Test-Chrombeschichtung von Borofloat-Substraten als Haftvermittlerschicht, 2. Goldbeschichtung von BorofloatSubstraten für den Einsatztest in einem spektroskopischen Ellipsometer im infraroten Spektralbereich.

SVO-Graphen: Graphene and Anti-reflective Coatings using Pulsed High-Current Arc Evaporation

\begin{tabular}{|l|l|}
\hline $\begin{array}{l}\text { Projektleiter } \\
\text { Mittelgeber }\end{array}$ & Prof. Dr. rer. nat. habil. Sigurd Schrader \\
\hline Projektvolumen & $188.914 €$ \\
\hline Laufzeit & $08 / 2016-07 / 2018$ \\
\hline
\end{tabular}

Ziel des hier skizzierten Vorhabens ist die Entwicklung eines neuartigen Verfahrens zur Abscheidung von schwarzen kohlenstoffbasierten Schichten und Graphen. Die prototypische Labormusteranlage soll in der Lage sein, Proben unterschiedlicher Größe und Beschaffenheit zu schwärzen, so dass sowohl die direkte als auch diffuse Reflexion deutlich vermindert wird. Ferner soll durch geeignete Modifikation der Anlage die Beschichtung von unterschiedlichen Substraten (Metalle, Isolatoren) mit Graphen untersucht werden. 
TRSA: Simultane Zwei-Stokes-Linien-Raman-Verstärkung in Kristallen

Projektleiter

Prof. Dr. rer. nat. habil. Sigurd Schrader

Mittelgeber

DFG

Projektvolumen

$217.520 €$

Laufzeit

04/2017-03/2020

Das Hauptziel des Projektes ist die Untersuchung und Optimierung simultaner Raman-Verstärkung der 1. und 2. StokesWelle (Two-Stokes Raman amplification) in einem kristallinen Raman-Verstärker, wenn der Strahlpropagationsfaktor M2 dieser Wellen am Verstärkereingang niedrige Werte aufweist. Es ist vorgesehen, dass die 1. und 2. Stokes-Welle mittels speziell entwickelter Raman-Generatoren erzeugt werden, wobei diese nahezu Beugungs-limitiert sein sollen (M-Faktor ca. 1.5 oder niedriger). 


\section{TABELLARISCHE ÜBERSICHT PUBLIKATIONEN}

Bauer JM, Heinrich F, Fursenko O, Steffen M, Blümich A, Pulwer S, Steglich P, Villringer C, Mai A, Schrader SK (2017)

Buchmann J, Guggenheim J, Scharfenorth C, Spannekrebs B, Villringer C, Laufer J (2017)

Burger M, Floris F, Cardone A, Farinola GM, Morandi V, Marabelli F, Comoretto D (2017)

Fursenko O, Lukosius M, Lupina G, Bauer JM, Villringer C, Mai A (2017)

Gerhard C (2017)

Hermann J, Grojo D, Axente E, Gerhard C, Burger M, Craciun V (2017)

Richter A, Anwand W, Chen C-L, Böttger R (2017)

Steglich P, Villringer C, Pulwer S, Dietzel B, Ksianzou V, Schrader S (2017)
Very high aspect ratio through silicon via reflectometry. Proceedings of SPIE 10329:103293J. doi: 10.1117/12.2269957

Characterization and modeling of Fabry-Perot ultrasound sensors with hard dielectric mirrors for photoacoustic imaging. Appl Opt 56(17):5039-5046. doi: 10.1364/AO.56.005039

Photo-induced absorption spectra of a poly(p-phenylenevinylene) polymer with fluorinated double bonds. Organic Electronics 43:214-221. doi: 10.1016/j.orgel.2017.01.023

Development of graphene process control by industrial optical spectroscopy setup. Proceedings of SPIE 10330:1033017. doi: 10.1117/12.2269603

Optics Manufacturing: Components and Systems, 1st. CRC Press, Boca Raton. ISBN: 978-1-4987-6459-9

Ideal radiation source for plasma spectroscopy generated by laser ablation. Physical Review E 96(5):53210. doi: 10.1103/PhysRevE.96.053210

Evaluation of defect formation in helium irradiated $\mathrm{Y} 2 \mathrm{O} 3$ doped $\mathrm{W}-\mathrm{Ti}$ alloys by positron annihilation and nanoindentation. Journal of Nuclear Materials 494:294-302. doi: 10.1016/j.jnucmat.2017.07.039

Chip-integrierte photonische Bauelemente. Wissenschaftliche Beiträge 21:55-59. doi: 10.15771/0949-8214_2017_7

Hybrid-Waveguide Ring Resonator for Biochemical Sensing. IEEE Sensors Journal (J-SEN) PP: 1-9. doi: 10.1109/JSEN.2017.2710318

\section{FORSCHUNGSPREISE}

Prof. Dr. rer. nat. habil. Sigurd Schrader
Forschungsprofessur 2014-2019 der TH Wildau
„Photonik, Laser- und Plasmatechnologien“ 


\section{Produktion und Material Production and Materials}




\section{Bio-Rohr: Entwicklung eines inhärent bioziden Materials und eines Verfahrens zur Rohrinnenbeschichtung von Trinkwasserrohren}

Prof. Dr. Michael Herzog

\author{
PROJEKTMITARBEITER \\ KOOPERATIONSPARTNER \\ PROJEKTVOLUMEN \\ MITTELGEBER \\ LAUFZEIT
}

\author{
Dipl.-Chem. E. Kornejew \\ TGA Rohrinnensanierung AG \\ $189.000 €$ \\ Bund | BMWi | ZIM Kooperationsprojekt \\ 10/2017-08/2019
}

Es ist das Ziel des Vorhabens, ein inhärent biozides Material, ein Reinigungsverfahren und ein Verfahren zur Durchführung einer Rohrinnenbeschichtung von Trinkwasserrohren nach den gegenwärtig gültigen Regeln zur Verfügung zu stellen. In Wasserleitungen aus Kupfer oder Stahl, ob in Warm- oder Kaltwasserleitungen, kann es bereits nach kurzer Zeit zu Schmutzablagerung, Keimbildung oder Lochfraß kommen. Es entstehen erhebliche Einschränkungen der Wohnqualität sowie gesundheitliche Risiken, die es unbedingt zu vermeiden gilt. Sauberes Wasser, das die Wasserwerke liefern und wir zur Erhaltung unserer Gesundheit benötigen, fließt nur aus intakten und sauberen Rohrleitungen. Die bisherige Lösung bestand im Austausch der betroffenen Rohre oder des kompletten Leitungsnetzes. Neuere Lösungen gehen von einer Innenbeschichtung der Rohre aus, wobei auch hier die Keimbildung überwacht werden muß. Die Beschichtung erfolgte bisher fast ausschließlich mit Epoxydharzen auf der Basis von Bisphenol-A-diglycidethern. Diese Ep- oxydharze sind heute nicht mehr Stand der Technik im Korrosionsschutz. Auch zahlreiche Untersuchungen haben ergeben, dass die Abgabe von Bisphenol $A$ an das Trinkwasser durch seine Wirkungsweise analog zu Hormonen und erhöhten TOC-Werten (Toxic Organic Carbon) Krebs erregend ist. Ferner können Beschichtungen Nahrungsgrundlage für Mikroorganismen sein, wodurch ein Biofilm entsteht. Eine erforderliche thermische Desinfektion oberhalb $71{ }^{\circ} \mathrm{C}$ erfolgt praktisch immer im Grenzbereich der thermischen Stabilität der Beschichtungen.

Durch die Einführung von stickstoffhaltigen Strukturen mit neuartigen Oligoharnstoffstrukturen in der Polymerkette sollen auslaugungsfeste biozide Epoxydharzsysteme auf einer speziell zu reinigenden Innenfläche mittels eines neuartigen Verfahrens aufgebracht werden.

KONTAKT

michael.herzog@th-wildau.de

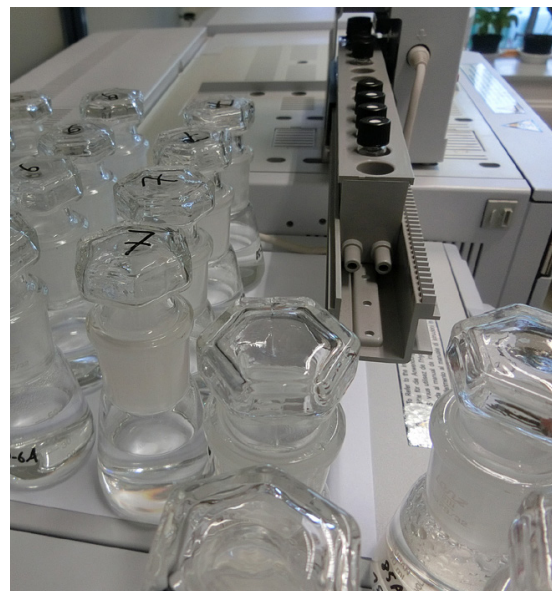

Abb. 1) Analyse der Migration von Inhaltsstoffen 


\section{METALCOAT}

Prof. Dr. Michael Herzog

PROJECT CONTRIBUTOR

COOPERATING PARTNERS

PROJECT CAPACITY

FUNDING AGENCY

DURATION
Dipl.-Chem. U. Knoll

Consortium with Partners from Germany and Bulgaria

Total Volume: $€$ 1.053.147 Share: TH Wildau: $€ 182.972$

EU | EUREKA | BMBF

07/2016-12/2018
The project aims at a novel type of coating system for the protection of steelwork and building materials, in particular outdoor equipment made of steel, galvanized steel or metal-coated steel, against environmental influences. The coating systems are based on recycled products (i.e. from the chemical recycling of flexible polyurethane foams), polythiols, and trimerized aliphatic diisocyanates and are designed to react with the surface atoms or oxidized products via two different mechanisms, thus forming chemical bonds between the metal surface and the coating.

By this and the refinement of the coating system to extremely low water absorption or water vapor and oxygen penetration, the metal surface is protected from the most aggressive environmental organisms. Further, the coatings are produced without the use of solvents, forming a very smooth surface that is very hard (Shore D hardness of $>80$ ) but elastic (elongation at break $>4 \%$ ). Such a coating composition is used to coat the steel items by a new process that combines spray coating with a UV curing process, following each other within a few seconds. Thus, a new technology with circular spraying and circular UV radiation is being developed.

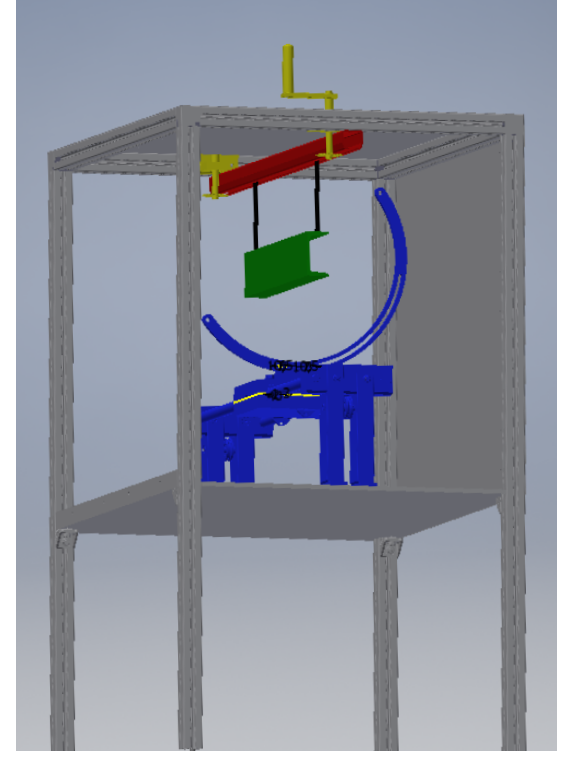

Fig. 1) Equipment construction

The main results are coated steel or metal products that resist corrosion, white corrosion and biocorrosion, including biological fouling by microorganisms for a prolonged time, i.e. at least ten years. The protection of steel or metal-coated steel by a further coating to maintain a non-changing surface without corrosion or biofilm adherence will constitute a major argument of marketing. The coated steel products manufactured by one of the partners are designed to be applied in construction, energy distribution, re-

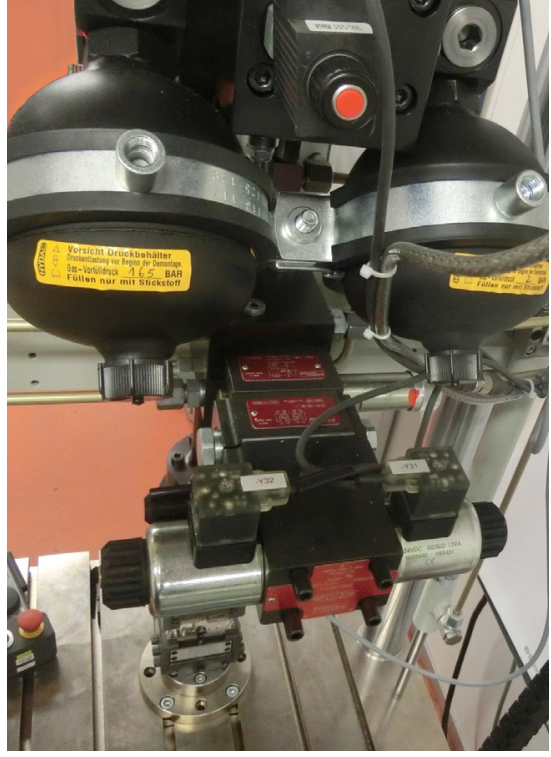

Fig. 2) Material testing machine

newable energy production (e.g. solar technology and offshore wind farms), in industrial structures as well as in consumer products such as fencing, trailers or tubing.

\section{CONTACT}

michael.herzog@th-wildau.de

GEFÖRDERT VOM

Bundesministerium

für Bildung

und Forschung 


\section{EffiProS: Durchführung von interaktiven Workshops "Efficient Production Systems"}

Prof. Dr.-Ing. Jörg Reiff-Stephan

PROJEKTMITARBEITER

MITTELGEBER

LAUFZEIT
Ron van de Sand M.Eng.

Wirtschaft

10/2017-09/2020
Im Rahmen des Projektes sollen die Prinzipien der Initiative Industrie 4.0 vermittelt und so der Wissenstransfer über die Hochschulgrenzen hinaus sichergestellt werden. Eines der wesentlichen Kernthemen ist hierbei die horizontale und vertikale Verkettung von Wertschöpfungsprozessen und die Implementierung von cyberphysischen Produktionssystemen. Neben der Auswertung von Daten steht deren Erhebung im Vordergrund, wobei vor allem der automatische Informationsaustausch von und zwischen Entitäten betrachtet wird.

Ziel der Veranstaltung ist das Vermitteln von Kenntnissen im Bereich der industriellen M2M-Kommunikation und das Erarbeiten des „Standes der Technik" in Bezug auf interoperable Ver- netzungsstrategien. Der Schwerpunkt liegt hierbei auf dem ganzheitlichen Datenaustausch von der Feldebene bis zur Cloud. In einem 2- bis 3-tägigen Workshop erlernen die Teilnehmer den Umgang mit den beiden Kommunikationsprotokollen OPC UA und MQTT und erarbeiten so die wesentlichen Begriffe rund um das Industrial Internet of Things. Auf der Grundlage eines demonstrativen Prozesses werden so Prozess- und Produktionsdaten abgebildet und ausgewertet.

Durch die Anwendung von interoperablen M2M-Protokollen können die Teilnehmer aktiv in den Prozess eingreifen und werden befähigt, Prozessparameter in Echtzeit zu verändern und zu verfolgen. Des Weiteren werden die Grundzüge der Datenspeicherung erläutert und anhand von Beispielaufgaben praktisch umgesetzt. Die TH-Wildau tritt hierbei als Akteur für den Wissenstransfer in Erscheinung und unterstützt Teilnehmer bei der Bewältigung von Herausforderungen im Zuge des Transformationsprozesses.

\section{KONTAKT}

joerg.reiff-stephan@th-wildau.de

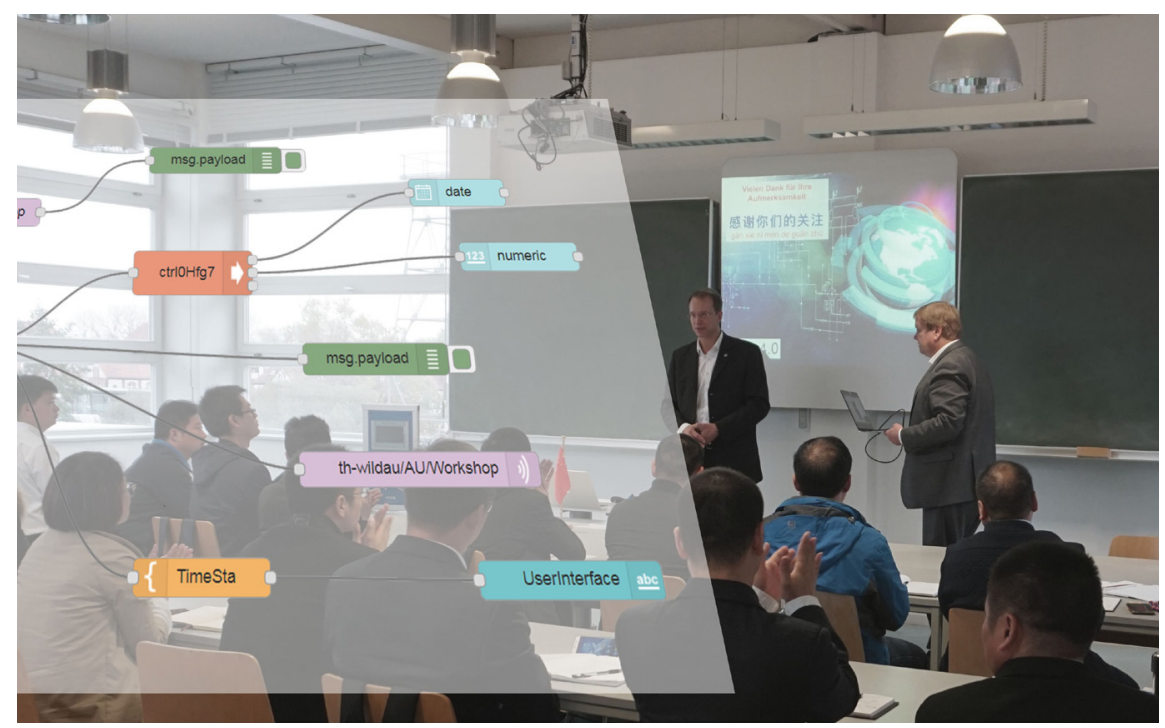




\section{LTA-Fit: Mittelstand-4.0-Kompetenzzentrum Cottbus - Teilvorhaben TH-Wildau - Produktionssystem, Logistik und Qualifizierung in der Cyberphysik}

Prof. Dr.-Ing. Jörg Reiff-Stephan

PROJEKTMITARBEITER

KOOPERATIONSPARTNER

PROJEKTVOLUMEN

MITTELGEBER

LAUFZEIT
Prof. Dr.-Ing. Frank Gillert, Norman Günther, Stephan Flatow, Ron van de Sand

HFB Fahrzeugbau, GeoSolar

$725.921 €$

Bund | BMWi

$11 / 2017-10 / 2020$
Das Mittelstand-4.0-Kompetenzzentrum Cottbus gehört zu Mittelstand-Digital. MitMittelstand-Digital unterstützt das Bundesministerium für Wirtschaft und Energie die Digitalisierung in kleinen und mittleren Unternehmen und im Handwerk. Übergeordnetes Ziel des Mittelstand-4.0-Kompetenzzentrums Cottbus ist die Erhöhung der digitalen Kompetenz in kleinen und mittelständischen Unternehmen (KMU) in Brandenburg, um die innovative Digitalisierung und Vernetzung in der brandenburgischen Wirtschaft zu fördern. Dadurch sollen die KMU und insbesondere die Kleinstunternehmen nicht nur in die Lage versetzt werden, ihre bestehenden Marktpositionen zu behaupten, sondern darüber hinaus auch weitere Geschäftsfelder und Märkte zu erschließen.

Insgesamt setzt sich das Kompetenzzentrum aus den Konsortialpartnern BTU-C/S, HNEE, IHP FF/O, IHK Cottbus und der THWi zusammen. Über die Projektdauer von drei Jahren (11/2017 bis 10/2020) wird ein Netzwerk zwischen Wissenschaft und Praxis aufge- baut, um den Wandel der Digitalisierung in Brandenburg zu unterstützen.

Auf der Basis der integrierten Expertise durch Prof. Dr.-Ing. Jörg Reiff-Stephan und Prof. Dr.-Ing. Frank Gillert mit den Forschungsgruppen „iC3@Smart Production" und "SichereObjektidentität" betreut der Konsortialstandort Wildau den Themenschwerpunkt „Digitalisierung in Logistik und Produktion“.

Im Rahmen des Projektes entwickelt das TH-Team ein Reifegradmodell zur Bestimmung des Digitalisierungs- und Qualifizierungsgrades von Unternehmen und deren Mitarbeitern. Durch die daraus resultierenden Ergebnisse und Sensibilisierungsmaßnahmen aller Konsortialpartner wird ein dreistufiges Qualifizierungsmodell (LTAFIT: Lernen - Training - Assistenz / Format, Instrument und Tools) entstehen. Die Qualifizierung ist dabei dreistufig angelegt, von niederschwelligen Inhalten (Level 1) bis zu komplexen fachspezifischen Inhalten (Level 3). Hierfür wird ein Planspiel entwickelt, das die Digitalisierungsmöglichkeiten und deren Potenziale pädagogisch veranschaulicht. Im Verlauf der Projektzeit werden zwei Umsetzungsprojekte mittels Lernpartnerschaften stattfinden, welche die Digitalisierung in Produktion und Logistik im Fokus haben und als Best-Practice-Beispiele zwischen Praxis und Wissenschaft in Brandenburg dienen.

KONTAKT

joerg.reiff-stephan@th-wildau.de

Gefördert durch 


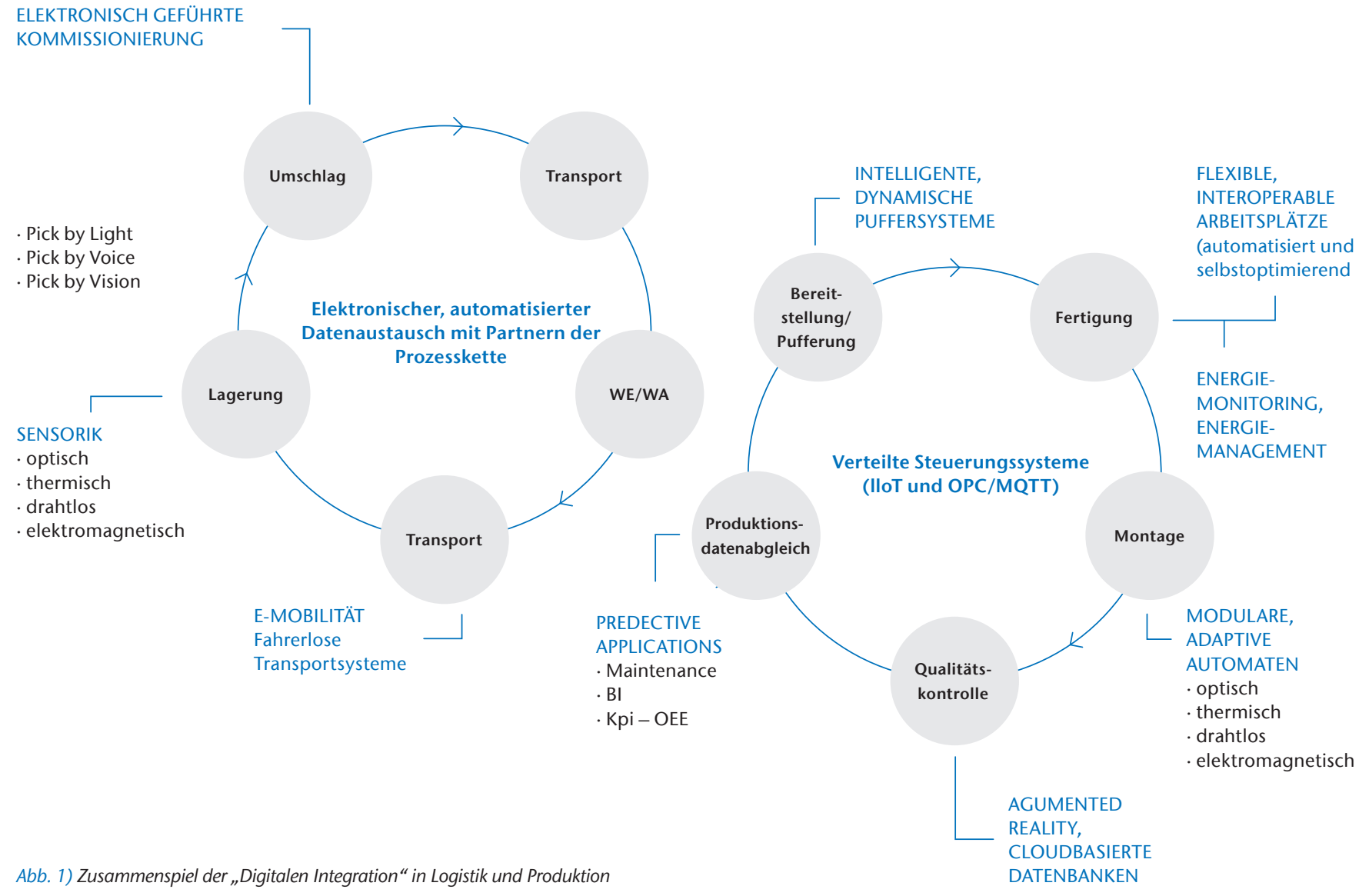




\section{SP-ICE: Machbarkeitsstudie eines automatisierten, auftragsindividuellen Lager- und Kommissioniersystems von Tiefkühlware}

Prof. Dr.-Ing. Jörg Reiff-Stephan

$\begin{array}{ll}\text { PROJEKTMITARBEITER } & \text { Jan Steimel } \\ \text { KOOPERATIONSPARTNER } & \text { IceGuerilla } \\ \text { PROJEKTVOLUMEN } & 3.000 € \\ \text { MITTELGEBER } & \text { ILB-FuE | BIG-Transfer klein } \\ \text { LAUFZEIT } & 03 / 2017-07 / 2017\end{array}$

Die IceGuerilla.de GmbH \& Co. KG ist ein kleines, mittelständisches Unternehmen in Beeskow (Brandenburg), das kundenindividuelles Speiseeis herstellt. Dabei können sich Kunden aus einem riesigen Sortiment an Zutaten ihr Lieblingseis zusammenstellen. Hierbei kann jeder der bestellten Eisbecher unterschiedliche Zutaten beinhalten. Die IceGuerilla.de GmbH \& Co. KG plant zu expandieren und möchte eine neue Produktionshalle bauen.

Im Rahmen des Projektes SP-ICE hat die Technische Hochschule Wildau, in Kooperation mit der IceGuerilla. de $\mathrm{GmbH} \&$ Co. KG, an einer Machbarkeitsstudie für ein automatisiertes, auftragsindividuelles Lager- und Kommissioniersystem im Tiefkühlbereich $\left(-24^{\circ} \mathrm{C}\right)$ gearbeitet.

Während der Projektbearbeitung erfolgte eine Ist- und Soll-Analyse. Auf dieser Basis wurden mehrere Konzeptvarianten erarbeitet und im Rahmen einer Nutzwertanalyse einander gegenübergestellt und bewertet. Hierfür kamen technische und wirtschaftliche Kriterien zur Anwendung. Des Weite- ren wurde für die IceGuerilla.de $\mathrm{GmbH}$ $\&$ Co. KG eine geeignete Konzeptvariante, eine Ausschreibungsunterlage (Lastenheft), erarbeitet. Das Ergebnis der Machbarkeitsstudie liefert eine allgemeingültige Aussage über die Eignung automatisierter, kundenindividueller Lager- und Kommissioniersysteme im Tiefkühlbereich für kleine und mittelständische Unternehmen.

\section{KONTAKT}

joerg.reiff-stepahn@th-wildau.de

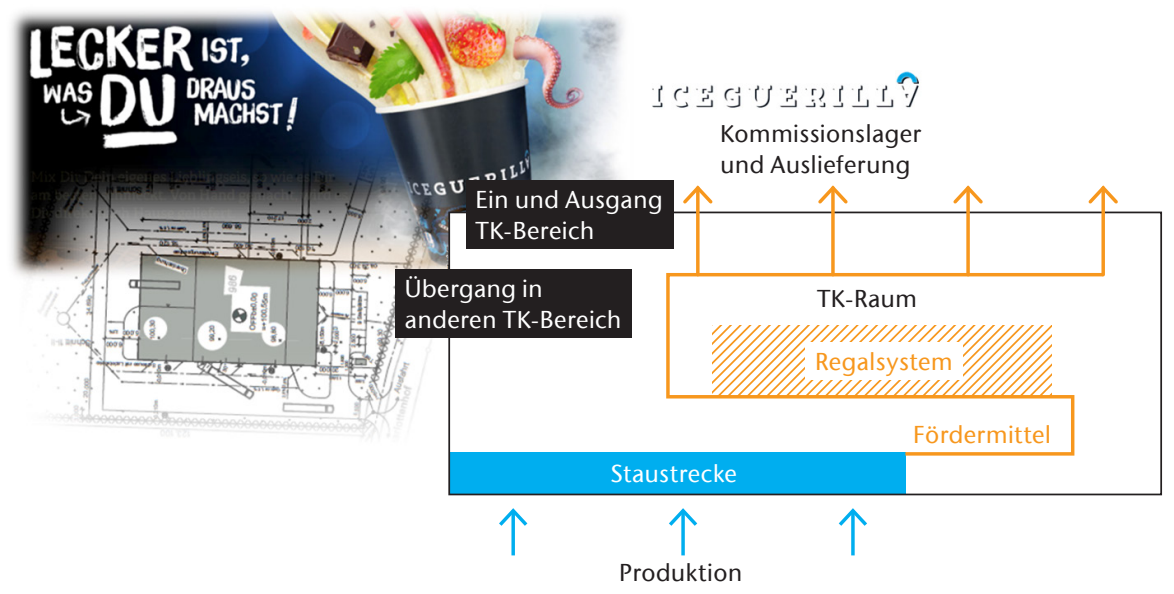

Abb. 1) Layoutplanung für ein automatisiertes TK-Kommissionierlager 


\section{xSCAD: Produktionsleitsysteme als Erweiterung von SCADA in der Automatisierungstechnik}

Prof. Dr.-Ing. Jörg Reiff-Stephan

PROJEKTMITARBEITER

PROJEKTVOLUMEN

MITTELGEBER

LAUFZEIT
Sebastian Schulz M.Eng.

$31.064 €$

Land Brandenburg | MWFK | EFRE | InfraFEI

02/2016-10/2017
Wirtschaftliche Produktionsprozesse sind in zunehmendem Maße aus dem Blickwinkel der Effektivität und Effizienz auf dem globalen Anbietermarkt zu steuern. Hierbei wird die Aufnahme und Verarbeitung von Informationen als einer der wichtigsten Innovationstreiber in der industrialisierten Produktionswelt angesehen. Das Internet der Dinge/Dienstleistungen/Daten (iD3) manifestiert sich hierbei in dem Szenario der „Industrie 4.0“-ProduktionsGesellschaft.

Die Erweiterung der Infrastruktur für Forschung, Entwicklung und Innovation an der TH Wildau durch das XSCADSystem APROL ermöglicht die flexible Einbindung steuerungstechnischer Funktionalitäten in eine Industrie-4.0Umgebung (WiTSI). Dazu wird ein effizientes cyberphysisches Produktionssystem (CPPS) als Grundentität die Ausprägung informationstechnischer Speicherglieder ausweisen, um eine horizontale wie auch vertikale Vernetzung interoperabel gewährleisten zu können. Ein Ansatz hierfür ist es, die Strukturen des OPC-UA serviceorientiert nutzbar zu machen. Im Rahmen

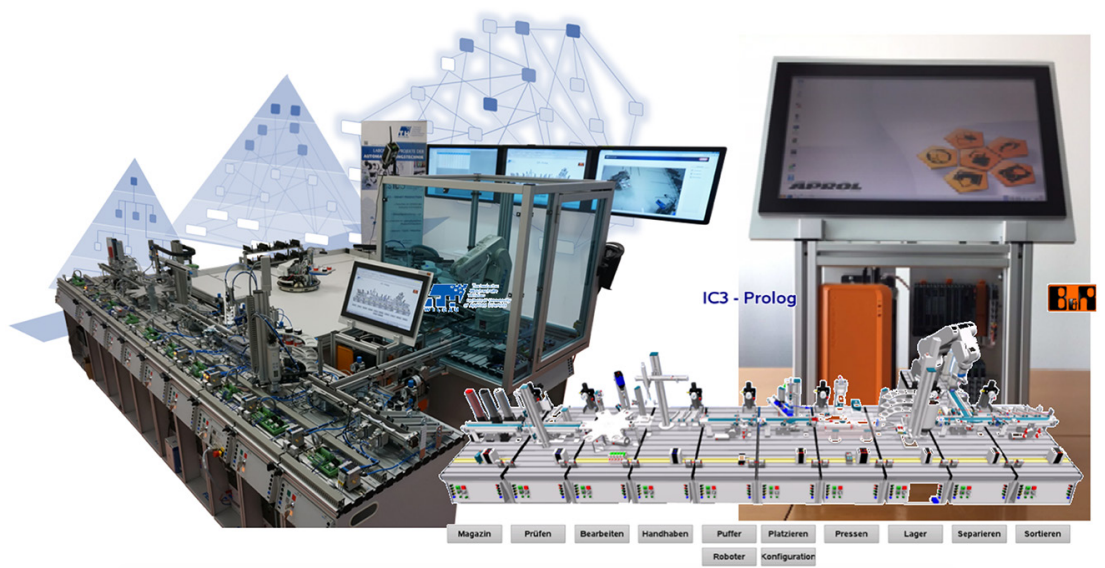

Abb. 1) Erweiterung der Prozesssteuerung für die i4.0-ProLog-Anlage

der Arbeiten ist ein Demonstrator entstanden, der diese informationstechnische Vernetzung ausbildet. Die weiterführende Anbindung an eine SQL-basierende Datenbankstruktur bereitet den Weg für die Interaktion mit weiteren Akteuren des CPPS. Eine Client-Server-Architektur erlaubt die flexible Einbindung weiterer Entitäten in das durchgängige Netzwerk. In der Zukunft kann eine Übermittlung mit einem auf dem TSN basierenden Netzwerkprotokoll die Echtzeitsta- bilität der Informationsverarbeitung garantieren und damit die Selbstorganisation unterstützen helfen. Hiermit wird ein Beitrag zu einer effizienten Produktionsweise mit durchgängiger, informationstechnischer Verknüpfung geleistet.

\section{KONTAKT}

joerg.reiff-stephan@th-wildau.de

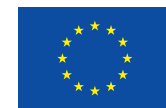

EUROPÄISCHE UNION Europäischer Fonds für Regionale Entwicklung

Investition in Ihre Zukunft!

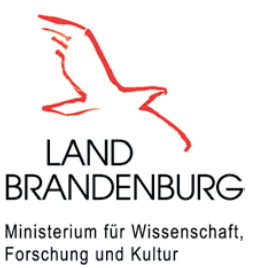

Ministerium für Wissenschaft,
Forschung und Kultur 


\section{TABELLARISCHE ÜBERSICHT LAUFENDER PROJEKTE}

\section{BIP - Bremsbelag Impedanz Test}

\begin{tabular}{|l|l|}
\hline Projektleiter & Prof. Dr. Peter Blaschke \\
\hline Mittelgeber & Bund | BMWi | ZIM \\
\hline Projektvolumen & $193.224 €$ \\
\hline Laufzeit & $04 / 2015-03 / 2017$ \\
\hline
\end{tabular}

Ziel des Projekts ist die Entwicklung eines neuartigen Messverfahrens einschließlich der zugehörigen Gerätetechnik für die Bestimmung der dynamischen Eigenschaften von Bremsbelägen in Abhängigkeit von Bremsdruck und Kolbenposition. Zum Projekt gehören Konzeption, Simulation und Sensitivitätsanalyse eines Bremsbelag Impedanz Tests (BIP) mit spezifischer Messprozedur und eingehender Validierungsergebnisse.

\section{ViKProQuO - Vibroakustische Composit- Prozesskontrolle für Qualitätssicherung und Optimierung}

\begin{tabular}{|c|c|}
\hline Projektleiter & Prof. Dr. Peter Blaschke \\
\hline Mittelgeber & Land Brandenburg | MWFK | EFRE | StaF \\
\hline Projektvolumen & $325.277 €$ \\
\hline Laufzeit & $07 / 2015-06 / 2018$ \\
\hline \multicolumn{2}{|c|}{$\begin{array}{l}\text { Das Ziel des Vorhabens ist die Entwicklung von praxisgerechten vibroakustischen Prüfverfahren für die strukturdynami- } \\
\text { sche Charakterisierung von Compositen. Durch diese innovativen Prüfverfahren können in der Herstellung von Leichtbau- } \\
\text { produkten Material, Energie und Ausschuss nachhaltig eingespart werden. Durch die Kooperation der Projektpartner TH } \\
\text { Wildau und Fraunhofer Pyco wird die technologische und anwendungsnahe Forschung am Standort Wildau insgesamt } \\
\text { nachhaltig gestärkt. }\end{array}$} \\
\hline
\end{tabular}

\section{ViTaMinB: Vibroakustikoptimierung mit der Transferpfadanalyse-Methode in Brandenburg}

\begin{tabular}{l|l}
\hline $\begin{array}{l}\text { Projektleiter } \\
\text { Mittelgeber }\end{array}$ & Prof. Dr. Peter Blaschke \\
\hline Projektvolumen & $46.400 €$ \\
\hline Laufzeit & $02 / 2016-04 / 2017$
\end{tabular}

Das Ziel des ViTaMinB-Projektes ist es, messtechnische Grundlagen für die strukturdynamische Charakterisierung von Composite-Materialien an Maschinen sowie Kraft-, Flug- und Schienenfahrzeugen zu schaffen. Für die Geräuschidentifikation ist es wichtig, die genauen Geräuschquellen und Klangursachen zu kennen und zusätzlich die Tranferpfade (Körper- bzw. Luftschall)zum Hörohr zu analysieren. Für diese Analyse wird die Transferpfadanalyse und -synthesetechnik (TPA/TPS) angewendet. 
Matre Mat: Modifizierung und Analyse von transparenten oder teil/-reflektierenden Materialien

Projektleiter

Prof. Dr. rer. nat. Andreas Foitzik, Prof. Dr. rer. nat. habil. Sigurd Schrader

Mittelgeber

Land Brandenburg | MWFK | EFRE | InfraFEI

Projektvolumen

$98.635 €$

Laufzeit

$10 / 2016-07 / 2018$

Das Ziel des Vorhabens ist die Entwicklung und Charakterisierung von modernen, transparenten oder teil-/reflektierenden Funktionsschichten und Oberflächen für den Einsatz in den Bereichen Mikrosystemtechnik, Photonik und Maschinenbau sowie aus oder polymer- oder glasartig hybriden oder metalloxidkeramischen Materialkombinationen. Die Funktionalisierung erfolgt durch Bearbeitung der Materialien mit Hilfe eines CO2 Laser und die Analyse mit Hilfe der Lasertriangulation.

B-Watch - Entwicklung eines sensorbasierten Bewegungsanalysesystems

Projektleiter Prof. Dr. rer. nat. Thomas Goldmann, Prof. Dr. phil. Bertil Haack

Kooperationspartner Charité Berlin, DreiF Informationstechnologie GmbH, SATIS INFORMATION GmbH

Mittelgeber $\quad$ Bund | BMWi | ZIM

\begin{tabular}{l|l} 
Projektvolumen $\quad 174.221 €$
\end{tabular}

Laufzeit $\quad$ 07/2014-06/2017

Im Rahmen der sensormotorischen Behandlung von Patienten mit Parkinson-Syndromen, Schlaganfall und anderen neurologischen Erkrankungen ist es erforderlich, Bewegungen präzise, objektiv und ohne hohen technischen bzw. zeitlichen Aufwand zu untersuchen. Beabsichtigt ist die Entwicklung eines sensorbasierten Systems zur Bewegungsanalyse im Prototypen insbesondere für die klinische Anwendung bei Parkinson- und Schlaganfall-Patienten, jedoch mit der Erweiterungsoption auf weitere Anwendungsbereiche.

Bio-Rohr: Entwicklung eines inhärent bioziden Materials und eines Verfahrens zur Rohrinnenbeschichtung von Trinkwasserrohren

\begin{tabular}{|l|l|}
\hline $\begin{array}{l}\text { Projektleiter } \\
\text { Mittelgeber }\end{array}$ & Prof. Dr. rer. nat. Michael Herzog \\
\hline Projektvolumen & $189.000 €$ \\
\hline Laufzeit & $10 / 2017-08 / 2019$ \\
\hline
\end{tabular}

In Wasserleitungen kann es bereits nach kurzer Zeit zu Schmutzablagerung und Keimbildung kommen. Es entstehen erhebliche Einschränkungen der Wohnqualität sowie gesundheitliche Risiken. Sauberes Wasser fließt nur aus intakten und sauberen Rohrleitungen. Ziel ist die Entwicklung eines inhärent biozides Materials, eines Reinigungsverfahrens und eines Verfahrens zur Durchführung einer Rohrinnenbeschichtung von Trinkwasserrohren. 
BridG iT! - Bridging the Gap through Intercultural Engineering Transposition!

Projektleiter

Prof. Dr. rer. nat. Michael Herzog

Kooperationspartner

Dedan Kimathi University of Technology Nyeri (Kenia)

Mittelgeber

DAAD

Projektvolumen

$200.000 €$

Laufzeit

$01 / 2014-12 / 2017$

Das Projekt Bridging the Gap through Intercultural Engineering Transposition! wird im Rahmen des Programms „Fachbezogene Partnerschaften mit Hochschulen in Entwicklungsländern” vom DAAD gefördert. Ausgangspunkt ist eine Hochschulkooperation zwischen der Dedan Kimathy University of Technology in Nyeri, Kenia, und der TH Wildau. Ziel des vierjährigen Projekts ist es unter anderem, einen Doppelabschluss Master im Maschinenbau zu etablieren sowie den Studentaustausch zu initiieren.

Entwicklung von UV-beständigen elastischen Vergussmassen mit guter Reparaturfähigkeit

\begin{tabular}{|c|c|}
\hline Projektleiter & Prof. Dr. rer. nat. Michael Herzog \\
\hline Mittelgeber & BUND | BMWi | ZIM \\
\hline Projektvolumen & $174.000 €$ \\
\hline Laufzeit & $01 / 2014-06 / 2017$ \\
\hline
\end{tabular}

Das Ziel dieses Vorhabens ist die Entwicklung neuartiger UV-beständiger Vergussmassen hoher Elastizität für die Anwendung in elektrischen Komponenten wie Verteilern, Schalter Kabelverbindern, Kabelmuffen etc., die im Falle einer Reparatur der betreffenden Komponente leicht entfernt werden können, d. h. dass sie trotz einer hohen Elastizität, die zur Aufnahme der Kabel und/oder Leitungen erforderlich ist, nur in geringem Maße an den verwendeten Materialien haften.

\section{Eurostars-Projekt CASENIPP: Isocyanatfreie Polycarbonat-Polyurethan-Systeme}

Projektleiter

Kooperationspartner Konsortium mit Partnern aus D, E, BG, HR

Mittelgeber

Projektvolumen

Laufzeit

Prof. Dr. rer. nat. Michael Herzog

EU | EUREKA | BMBF

$173.000 €$

07/2017-12/2019
Dieses Vorhaben ist auf die Entwicklung eines neuen Verfahrens zur Herstellung von isocyanatfreien Systemen für Schaumstoffe, Elastomere und Beschichtungen auf der Basis von Kohlendioxid-Produkten und Aminen auf der Basis nachwachsender Rohstoffe gerichtet. Als Rohstoffe sollen Polycarbonatbis(carbonate) eingesetzt werden. 
In-lining - Entwicklung eines neuen Materials und Verfahrens zum In-lining

Projektleiter

Prof. Dr. rer. nat. Michael Herzog

Mittelgeber

Bund | BMWi | ZIM

Projektvolumen

$190.000 €$

Laufzeit

07/2015-04/2017

Ziel des Vorhabens ist es, durch Kombination von positiv gelisteten Komponenten Formulierungen für den kalten und warmen Trinkwasserbereich auf der Basis von aminhärtenden Epoxydsystemen zu entwickeln und die Anforderungen an die Beschichtungspraxis in Bezug auf die Viskosität der Komponenten, die Topfzeit der Systeme und die Härtungszeit bei Umgebungstemperatur sowie die Eigenschaften der Schichten in Bezug auf Wasseraufnahme, Sauerstoffdiffusion, Festigkeit und Temeraturbeständigkeit zu erreichen.

Eurostars-Projekt METALCOAT: Steel products protected by Corrosion inhibiting, self-healing, solvent- and primer-less

Projektleiter Prof. Dr. rer. nat. Michael Herzog

Kooperationspartner Konsortium mit Partnern aus Deutschland und Bulgarien
Mittelgeber
EU | EUREKA | BMBF

Projektvolumen

$182.972 €$

Laufzeit

$07 / 2016-12 / 2018$

The project is directed to an new process to coat steel products including galvanized or metal coated steel products by solvent-less coating using a polymer coating material without the application of a primer made by rotational spray coating technology an immediately following a UV curing process to receive metal products smoothly coated by an elastic layer to be chemically bonded to the metal and showing extremely low water and oxygen penetration to avoid corrosion over a log time range.

\section{Research Stay Kenya}

\begin{tabular}{l|l} 
Projektleiter $\quad$ Prof. Dr. Michael Herzog \\
\hline
\end{tabular}

Mitarbeiter Herr Tanui

Kooperationspartner Dedan Kimathi University of Technology Nyeri (Kenia)

Mittelgeber DAAD

Projektvolumen $\quad 6.700 €$

Laufzeit $\quad$ 09/2017-11/2017

The scientific investigations in the field of burning processes proposed by Mr. Tanui fit into the research profile of our faculty of engineering and natural sciences and scientific cooperation by designated experts Prof. Mirre and Prof. Herzog is ensured. We appreciate the initiative of Mr. Tanui due to the fact that the expected results deliver experimental data for further progress in complex calculations for the simulation of burning processes at solid fuels. 


\section{STIBET - Förderung ausländischer Doktoranden aus Partnerhochschulen}

Projektleiter

Mittelgeber

Projektvolumen

Laufzeit

Prof. Dr. rer. nat. Michael Herzog

DAAD

$15.600 €$

$01 / 2016-12 / 2018$

Dieses Projekt dient der Betreuung ausländischer Doktoranden an der TH Wildau.

Eurostars-Projekt Doublelayer: Untersuchung neuartiger Gele und Hartschalenmaterialien

Projektleiter

Prof. Dr. rer. nat. Michael Herzog

Kooperationspartner

Konsortium mit Partnern aus D, F, und SK

Mittelgeber

EU | EUREKA | BMBF

Projektvolumen

$120.850 €$

Laufzeit

$10 / 2015-03 / 2018$

Bei diesem Projekt handelt es sich um ein Teilprojekt des Verbundprojektes zur „Entwicklung von zweilagig isolierend und leitenden Kabelverbindungen, Verzweigungen und Anschlüssen“. Es sollen für diesen Zweck neuartige Gele und Hartschalenmaterialien untersucht werden.

\section{UP Harz: Entwicklung UP-Harz Recycling}

Projektleiter

Mittelgeber

Projektvolumen

Laufzeit
Prof. Dr. rer. nat. Michael Herzog

Bund | BMWi | ZIM Kooperationsprojekt

$190.000 €$

$12 / 2016-12 / 2018$

Ziel des Vorhabens ist ein Verfahren und eine Anlage zum chemischen Recycling auf der Basis gefüllter ungesättigter Polyesterharze zu entwickeln. Bei der Herstellung von Formteilen auf der Basis von UP-Harzen, die mit parikulären Verstärkungsstoffen gefüllt sind, fallen zwangsläufig Abfälle in Form von Angüssen, Graten oder Ausschussteilen an. Beim Partner GTK liegen die Mengen im Bereich von $8 \%$ bis $12 \%$ des Produktionsvolumens. Diese Mengen werden zur Zeit nicht wiederverwertet.

\section{Zugproben}

Projektleiter

Prof. Dr. rer. nat. Michael Herzog

Mittelgeber

\section{Wirtschaft}

Laufzeit

$11 / 2015-01 / 2018$

Bestimmung mechanischer Eigenschaften: Durchführung von Zugversuchen an Kunststoff-Standardflachproben inkl. grafischer Auswertung. 
RevDop: Entwicklung strömungsoptimierter Bauteile im Automotivebereich

\begin{tabular}{|l|l|}
\hline Projektleiter & Prof. Dr.-Ing. Stefan Kubica \\
\hline Mittelgeber & Bund | BMWi | ESF | EXIST \\
\hline Projektvolumen & $138.600 €$ \\
\hline Laufzeit & $08 / 2016-07 / 2017$ \\
\hline
\end{tabular}

RevDop führt Technologieoptimierungen von Strömungsbauteilen für Verbrennungsmotoren durch, um eine Erhöhung des Wirkungsgrades zu erzielen. Somit wird gleichzeitig die Motorleistung gesteigert, der Kraftstoffverbrauch gesenkt und darüber hinaus der Schadstoffausstoß minimiert. Es werden also Zielstellungen für Kosteneinsparungen, nachhaltige Ökologie und Leistungssteigerungen gleichzietig erreicht.

\section{CFD - Flowmeter}

\begin{tabular}{l|l} 
Projektleiter & „Prof. Dipl.-Ing. Thomas Mirre \\
\hline Kooperationspartner & Wirtschaft \\
\hline
\end{tabular}

\section{Mittelgeber $\quad$ EEE Anlagenbau $\mathrm{GmbH}$}

Laufzeit $\quad 11 / 2017$

Simulation des Druckverlaufes eines vorgegebenen Mediums bei unterschiedlichen Ströumungsgeschwindigkeiten und einer gegebenen Rohrkonstruktion mit Bogen, in welche ein Flowmeter eingebunden ist, sowie mit kurzem, geradem Ein- und Auslauf für eine Geschwindigkeit

\section{CFD - Simulationstechnik/CFD/FEM}

Projektleiter

Laufzeit
Prof. Dipl.-Ing. Thomas Mirre

09/2015-08/2018

Das Projekt ermöglicht in verschiedenen Bereichen mit ingenieurmäßigen Methoden der CFD und FEM Bauteil- und Prozesssimulationen durchzuführen, um erforderliche Berechnungen zu Festigkeit, Strömungen, Wärmeübertragungen u. ä. umzusetzen.

\section{EffiProS: Durchführung von interaktiven Workshops „Efficient Production Systems“}

Projektleiter

Mittelgeber

Laufzeit
Prof. Dr.-Ing. Jörg Reiff-Stephan

Wirtschaft

$10 / 2017-09 / 2020$

Ziel des Projektes ist das Vermitteln von Kenntnissen im Bereich der industriellen M2M Kommunikation und das Erarbeiten des „Stand der Technik“ in Bezug auf interoperable Vernetzungsstrategien. Der Schwerpunkt liegt hierbei auf dem ganzheitlichen Datenaustausch von der Feldebene bis zur Cloud. In einem 2-3 tägigen Workshop erlernen die Teilnehmer den Umgang mit den beiden Kommnikationsprotokollen OPC UA und MQTT und erarbeiten so die wesentlichen Begriffe rund um das Industrial Internet of Things. Auf Grundlage eines demonstrativen Prozesses werden so Prozess- und Produktionsdaten abgebildet und ausgewertet. Durch die Anwendung von interoperablen M2M Proto-kollen können die Teilnehmer aktiv in den Prozess eingreifen und werden befähigt Prozessparameter in Echtzeit zu verändern und zu verfolgen. 
Projektleiter

Kooperationspartner

Mittelgeber

Projektvolumen

Laufzeit
Prof. Dr.-Ing. Jörg Reiff-Stephan

Brandenburgische Technische Universität Cottbus - Senftenberg

Hochschule für Nachhaltige Entwicklung Eberswalde

Landesarbeitsgemeinschaft der Industrie- und Handelskammern IHP GmbH Leibnitz-Institut für innovative Mikroelektronik

Bund | BMWi

$725.921 €$

$11 / 2017-10 / 2020$

Als Projektpartner im Verbundprojekt „LTA4.0“ zum Aufbau eines Brandenburger Kompetenzzentrums im Bereich „Industrie4.0“ wird die Technische Hochschule Wildau Demonstratoren „Logistik/Produktionstechnik“ aufbauen und aus-/weiterbildungsrelevante Inhalte entwerfen und vermitteln. Ziel ist es, für KMU eine Basis zu schaffen, sich mit den Inhalten der zukünftigen Wirtschaftssysteme vertraut zu machen und Mitarbeiter wie auch Führungskräfte anwendungsbezogene Weiterbildungsangebote zu schaffen.

\section{MetamoFAB: Metamorphose zur intelligenten und vernetzten Fabrik}

Projektleiter Prof. Dr.-Ing. Jörg Reiff-Stephan

Kooperationspartner SIEMENS, FESTO, infineon, Universität Potsdam LSWi, Fraunhofer IPK, Pickert, budatec

Mittelgeber $\quad$ BMWi

Laufzeit 12/2013-03/2017

Mit den Prinzipien der Initiative Industrie 4.0 soll ein Beitrag zur Zukunftssicherung des Produktionsstandorts Deutschland geleistet werden. Im Vordergrund steht hierbei die umfangreiche Vernetzung von Wertschöpfungsprozessen bis hin zum cyberphysischen Produktionssystem. Unter Berücksichtigung bestehender Strukturen muss die Realisierung der Industrie 4.0-Bestrebungen durch einen schrittweisen Transformationsprozess erfolgen. Im Rahmen des Forschungsprojektes MetamoFAB wird nach Wegen gesucht, welche Betrieben eine derartige Metamorphose zur intelligenten und vernetzen Fabrik ermöglichen. Ziele des Projektes sind zum einen die Entwicklung von fallspezifischen Referenzmodellen und zum anderen die Ableitung von erforderlichen Fähigkeiten der beteiligten Entitäten, sowie neuer Regeln für deren Zusammenwirken. Ein weiterer Schwerpunkt des Projekts ist die Entwicklung eines Transformationscockpits um Entscheidungen, welche in stark vernetzten Systemen getroffen werden, vor der Realisierung virtuell absichern zu können. Zusätzlich sollen gemeingültige Methoden und Werkzeuge für die Planung, Durchführung und Betreuung der Transformation zur intelligenten Fabrik entstehen. Die entwickelten Vorgehensweisen und Werkzeuge werden schließlich anhand virtueller und realer Demonstratoren validiert. An dem Forschungsprojekt ist ein breites Konsortium an namenhaften Industriebetrieben und Hochschulen beteiligt. Die TH Wildau tritt hierbei als Assoziierter Forschungspartner in Erscheinung und unterstützt das Konsortium vor allen bei der Erstellung der Referenzmodelle und Umsetzung der Demonstratoren. Im Fokus stehen dabei vor allem die Nutzbarmachung bestehender Kommunikations- und Sensortechnologien für die Realisierung intelligenter Fabriken. 
TelhydrA: Entwicklung eines teleskophydraulischen Aufzugsystems

Projektleiter Prof. Dr.-Ing. Jörg Reiff-Stephan

Kooperationspartner Grädler Fördertechnik GmbH

Mittelgeber

Bund | BMWi | ZIM

Projektvolumen

$188.632 €$

Laufzeit

$01 / 2016-12 / 2017$

Ziel des geplanten FuE-Kooperationsprojektes zwischen der Grädler Fördertechnik GmbH (GFT) und der TH Wildau ist die Entwicklung eines innovativen hydraulischen Aufzugsystems. Innerhalb des Projektes wird GFT die Hydraulikzylinder, das Führungssystem sowie die Aufzugssteuerung entwicklen. Aufgabe der TH Wildau ist die Entwicklung des hydraulischen Antriebssystems sowie der Hydrauliksteuerung.

SP-ICE: Machbarkeitsstudie eines automatisierten, auftragsindividuellen Lager- und Kommissioniersystems von Tiefkühlware

\begin{tabular}{|l|l|}
\hline Projektleiter & Prof. Dr.-Ing. Jörg Reiff-Stephan \\
\hline Kooperationspartner & IceGuerilla.de GmbH \& Co KG \\
\hline Mittelgeber & FuE I BIG-Transfer klein \\
\hline Projektvolumen & $3.000 €$ \\
\hline Laufzeit & $03 / 2017-07 / 2017$ \\
\hline
\end{tabular}

Machbarkeitstudie eines automatisierten Lager- und Kommissioniersystems für Tiefkühlware. Es handelt sich um eine kundenindividuelle Produktion von Speiseeisprodukten. Wesentliche Zielsetzung ist die Auslegung des automatisierten Prozesses zum Ein- und Auslagern anhand der Vorgaben cyberphysischer Produktionssysteme.

xSCAD: Produktionsleitsysteme als Erweiterung von SCADA in der Automatisierungstechnik

\begin{tabular}{|l|l|}
\hline Projektleiter & Prof. Dr.-Ing. Jörg Reiff-Stephan \\
\hline Mittelgeber & Land Brandenburg | MWFK | EFRE | InfraFEI \\
\hline Projektvolumen & $31.064 €$ \\
\hline Laufzeit & $02 / 2016-10 / 2017$ \\
\hline
\end{tabular}

Mit der Entwicklung der Forschungsstrategie „Industrie 4.0“ wird es wesentlich werden, Produktionsprozesse intelligent zu führen und stetig einen Überblick über die Produktionsentwicklung zu haben. Forschungsaufgaben in diesem Umfeld zielen auf die Weiterentwicklung von SCADA Systemen, um eine selbstanalysierende und -optimierende Produktion zu erreichen. Mit der Anschaffung des Antragsgegenstandes wird die F\&E in diesem Bereich an der Hochschule nachhaltig unterstützt. 
Innovative Energiesysteme: Phase 2

\begin{tabular}{l|l}
$\begin{array}{l}\text { Projektleiter } \\
\text { Mittelgeber }\end{array}$ & $\begin{array}{l}\text { Prof. Dr. Siegfried Rolle } \\
\text { Bund | BMWi | ZIM }\end{array}$ \\
\hline $\begin{array}{l}\text { Projektvolumen } \\
\text { Laufzeit }\end{array}$ & $053.491 €$ \\
\hline $\begin{array}{l}\text { Die Entwicklung von Kompetenzen innovativer Engeriesysteme bis hin zu einem regenerativ-ambivalenten Kraftwerk auf } \\
\text { der Basis regenerativer Energieerzeuger und Energiewandler in Verbindung mit neuen effizienten Speichereinheiten ist } \\
\text { das Ziel des Netzwerkes aus Wirtschaft und Wissenschaft. Die Herausforderung in der gemeinsamen Arbeit liegt in der } \\
\text { standortgerechten und verbrauchsorientierten Dimensionierung und Steuerung der Einzelkomponenten. }\end{array}$
\end{tabular}

\section{HASA: Hocheffiziente Absorptionswärmepumpe mit skalierbarem Absorbens}

Projektleiter

Prof. Dr. rer. nat. Franz-Xaver Wildenauer

Mittelgeber

Bund | BMBF

Projektvolumen

$272.376 €$

Laufzeit

$$
11 / 2016-10 / 2019
$$

Das Ziel ist die Entwicklung einer hocheffizienten Adsorptionswärmepumpe mit dem skalierbaren Sorptionsmaterial DAY Zeolith, optimiert für die Kälte- und Wärmebereitstellung in Industrie und Wirtschaft. Für das Adsorptionsmaterial ist die Entwicklung eines Herstellungsprozesses im Drehrohrofen für kurze Durchlaufzeiten vorzunehmen. Hierbei sollen die Materialparameter erzielt werden, die einer passgenauen Einstellung auf das neue Anlagenkonzept der Adsorptionswärmepumpe entsprechen. 


\section{TABELLARISCHE ÜBERSICHT PUBLIKATIONEN}

Alarcón D, Sampathkumar KR, Kamenzky R, Mallareddy TT, Sven Angermann S, Frahm A, Rüther-Kindel W, Blaschke P (2017)

Blaschke P, Schneider S, Kamenzky R, Alarcón D (2017)

Dimitrov K, Todorova D, Nenkova S, Herzog M (2017)

Geißler U, Schneider-Ramelow M (2017)

Geleç E, Kern M, Schneider B, Ullrich A, Vladova G, Gronau N, Lipinski R von, Buße D, Oertwig N (2017)

Kamenzky R, Schneider T, Schneider S, Blaschke P (2017)

Knothe T, Reiff-Stephan J, Vladova G, Ullrich A, Lipinski R von, Buße D, Kern M (2017)

Köll R, van Helden W, Engel G, Wagner W, Dang B, Jänchen J, Kerskes H, Badenhop T, Herzog TH (2017)

Langat HK, Dimitrov K, Herzog M, Muchiri P, Keraita J (2017)

Lisker R, Hellwig U, Wildenauer F-X (2017)
Modal Model Validation Using 3D SLDV, Geometry Scanning and FEM of a Multi-Purpose Drone Propeller Blade. 2. In: Di D. Maio PC((ed) Rotating Machinery, Hybrid Test Methods, Vibro-Acoustics \& Laser Vibrometry, Vol. 8, 1st edn. Conference Proceedings of the Society for Experimental Mechanics Series. Springer International Publishing, Garden Grove, CA, USA, ISBN: 978-3-319-54647-6, pp 13-22. doi: 10.1007/978-3-319-54648-3_2

Non-Linearity Identification of Composite Materials by Scalable Impact Modal Testing. In: Wee Sit E, Walber C, Walter P, Seidlitz S (eds) Sensors and Instrumentation, Vol. 5, 1st edn. Conference Proceedings of the Society for Experimental Mechanics Series. Springer International Publishing, Garden Grove, CA, USA, ISBN: 978-3-319-54986-6, pp 7-14. doi: 10.1007/978-3-319-54987-3_2

Polycarbonate diols to produce elastic polyurethane foams - a method of immobilization of carbon dioxide into a polymer structure. Journal of Chemical Technology and Metallurgy 52(1):28-40

Aluminium-Scandium als alternative Bond-Pad-Chip-Metallisierung für Leistungshalbleiter. PLUS : Produktion von Leiterplatten und Systemen 2017(1):92-94

Metamorphose zur intelligenten und vernetzen Fabrik. In: Weinert N, Plank M, Ullrich A (eds) Metamorphose zur intelligenten und vernetzten Fabrik: Ergebnisse des Verbundforschungsprojekts MetamoFAB. Springer Berlin Heidelberg, Berlin, Heidelberg, ISBN: 978-3-662-54317-7, pp 39-120. doi: 10.1007/978-3-662-54317-7_3

LEx - Levitation and Excitation Method for Operational Modal Analysis. In: 7th IOMAC: International Operational Modal Analysis Conference. Shaker, Aachen, ISBN: 978-3-8440-5247-3, pp 275-278

Industrie 4.0 im Produktionsumfeld. In: Weinert N, Plank M, Ullrich A (eds) Metamorphose zur intelligenten und vernetzten Fabrik: Ergebnisse des Verbundforschungsprojekts MetamoFAB. Springer Berlin Heidelberg, Berlin, Heidelberg, ISBN: 978-3-662-54317-7, pp 5-37. doi: 10.1007/9783-662-54317-7_2

An experimental investigation of a realistic-scale seasonal solar adsorption storage system for buildings. Solar Energy 155:388-397. doi: 10.1016/j.solener.2017.06.043

Investigating the Thermal and Mechanical Performance of Polylactic Acid (PLA) Reinforced with cellulose, wood fibers and Copolymer. IOSR Journal of Polymer and Textile Engineering (IOSR-JPTE) 4(3):25-32. doi: 10.9790/019X-04032532

Thin Film Condensation in a Tesla Turbine. Wissenschaftliche Beiträge 21:71-76. doi: 10.15771/0949-8214_2017_9 
Mallareddy TT, Alarcón D, Schneider S, Edel C-H, Blaschke P (2017)

Mallareddy TT, Blaschke P, Schneider S, Alarcón D (2017)

Matev A, Dimitrov K, Döhler T, Velev P, Herzog M (2017)

Matev A, Velev P, Herzog M (2017)

Miersch N, Roßmann R, Holz C (2017)

Moritz S, Schulz S, Reiff-Stephan J (2017)

Reiff-Stephan J (2017)

Reiff-Stephan J (2017)

Reiff-Stephan J (2017)

Reiff-Stephan J (2017)

Reiff-Stephan J, Vladova G, Ullrich A (2017)
Identification of Pressure-Dependent Modal Properties of Non-Linear Brake Pads by Using Scalable Dirac Impulse. In: Kerschen G (ed) Nonlinear Dynamics, Vol. 1. Proceedings of the 35th IMAC, A Conference and Exposition on Structural Dynamics 2017, 1st edn. Conference Proceedings of the Society for Experimental Mechanics Series. Springer International Publishing, Garden Grove, CA, USA, ISBN: 978-3-319-54403-8, pp 99-106. doi: 10.1007/978-3-319-54404-5

Brake Impedance Test Stand - Modal Testing of Brake Pads under Pressurized Condition Using 3D SLDV and a Scalable Automatic Modal Hammer. SAE Technical Paper 2017-01-2485. \#EB2017-VDT-013

Investigation On The Possibility Of Using Water-Based Polyurethane Acrylates In The Production Process Of Fiberglass Reinforced Composite Materials. IOSR Journal of Polymer and Textile Engineering (IOSR-JPTE) 4(2):29-38. doi: 10.9790/019X-04022938

An investigation on the possibility of fiberglass manufacture using a matrix obtained from water diluted mixtures of unsaturated lyester and polyurethane resins. Journal of Chemical Technology and Metallurgy 52(5):852-858

Grundlagenuntersuchung zu ausgewählten finiten Elementen der Strukturmechanik für ein einfaches validierbares FE-Modell. Wissenschaftliche Beiträge 21:61-70. doi: 10.15771/0949-8214_2017_8

Entwurf einer Datenbankstruktur zur Erfassung von Messdaten eines cyberphysischen Greifsystems. In: Reiff-Stephan J (ed) Tagungsband AALE 2017. VDE VERLAG, Berlin•Offenbach, ISBN: 978-3-8007-4360-5, pp 141-149

Der Mensch als Entität im cyberphysischen Produktionssystem. . In: ReiffStephan J (ed) Tagungsband AALE 2017. VDE VERLAG, Berlin•Offenbach, ISBN: 978-3-8007-4360-5, pp 293-299

Tagungsband AALE 2017. VDE VERLAG, Berlin, Offenbach. ISBN: 978-38007-4360-5

Tagungsband AALE 2017. Das Forum für Fachleute der Automatisierungstechnik aus Hochschulen und Wirtschaft. In: Reiff-Stephan J (ed) Tagungsband AALE 2017. VDE VERLAG, Berlin · Offenbach, ISBN: 978-38007-4360-5

Virtuelle Steuerung eines verteilten Energiemanagements. Wissenschaftliche Beiträge 21: 77-81. doi: 10.15771/0949-8214_2017_10

Der Mensch im Umfeld von Industrie 4.0. In: Weinert N, Plank M, Ullrich A (eds) Metamorphose zur intelligenten und vernetzten Fabrik: Ergebnisse des Verbundforschungsprojekts MetamoFAB. Springer Berlin Heidelberg, Berlin, Heidelberg, ISBN: 978-3-662-54317-7, pp 23-30 


\section{KOOPERATIVE PROMOTIONEN}

Andrey Matev
Glasfaserverstärkte Kompositmaterialien auf der Basis wässriger Polyurethanacrylat Dispersionen
Prof. Dr. rer. nat. Michael Herzog
Universität für Chemische Technologie und Metallurgie Sofia 
Verkehr und Logistik Transport and Logistics 


\title{
DynaNetz: Dynamisches, individualisiertes Angebotsnetz im Öffentlichen Personennahverkehr
}

\author{
Prof. Dr. Jens Wollenweber (Prof. Dr.-Ing. Herbert Sonntag)
}

PROJEKTMITARBEITER

KOOPERATIONSPARTNER

PROJEKTVOLUMEN

MITTELGEBER

LAUFZEIT
Dipl.-Ing. Philip Michalk

Verkehrsverbund Berlin Brandenburg, IVU Traffic Technologies AG, Door2Door $\mathrm{GmbH}$, Verkehrsbetriebe Oberhavel

$118.483 €$

Bund | BMBF

$12 / 2017-11 / 2018$
ÖPNV-Angebotsnetze sind in aller Regel statisch: Die Haltestellen, Routenverläufe und Abfahrtszeiten sind langfristig geplant und vorgegeben. Fahrzeuge sind in Randzeiten häufig nicht und in Stoßzeiten zu stark ausgelastet. Fahrgäste gelangen selten auf der direkten Route zum Ziel: Sie können sich nur innerhalb des starren Angebotsnetzes bewegen und dieses von ihrem Ausgangspunkt zum Zielort abfahren.

Hier setzt DynaNetz an: Teile eines vorhandenen städtischen ÖPNV-Angebotsnetzes werden dynamisch und bedarfsgerecht anhand individueller Nutzeranfragen in Echtzeit neu geplant und mit elektrischen Kleinbussen bedient.

Der Fahrgast gibt (wie heute bereits üblich) seinen aktuellen Standort und seinen Zielort in eine App ein. Das DynaNetz-System plant die schnellstmögliche Reiseroute, bezieht dabei aber auch Kleinbusse ein, die bedarfsgerecht eingesetzt werden. Die Kleinbusse werden dabei (anders als klassische Taxidienste) nicht exklusiv für eine Fahrgastanfrage eingesetzt, sondern dynamisch anhand verschiedener Fahrgastanfragen geroutet. Die bedarfsgerecht geplante Route wird dabei mithilfe eines multikriteriellen Ansatzes im Hinblick auf Fahrzeugauslastung, Auslastung der ansonsten verkehrenden Linienangebote und schnellste Reiseroute für den Fahrgast optimiert. Dem Fahrgast wird die Nutzung eines DynaNetz-Fahrzeuges nur dann angeboten, wenn ein entsprechendes Angebot gerade verfügbar ist, und er erhält in diesem Fall die Möglichkeit, eine individualisierte Reise zu buchen.

Damit ist nicht nur ein deutlich effizienterer Fahrzeugeinsatz möglich, sondern auch ein stärker individualisiertes und attraktives ÖPNV-Angebot. Darüber hinaus erlaubt ein digitalisiertes Angebot auch die problemlose Verknüpfung mit anderen Mobilitätsformen, die ebenfalls Teil des DynaNetz-Ansatzes ist. Durch die Dynamisierung wird der Einsatz kleinerer Fahrzeuge möglich. Durch den Einsatz elektrischer Kleinbusse wird der Schadstoffausstoß minimiert.
Durch die Verknüpfung mit anderen Mobilitätsangeboten kann einerseits ein schnelles und an dem Kundenbedarf orientiertes Reiseangebot kreiert werden, andererseits wird die Anzahl der urbanen Fahrzeugbewegungen auf ein notwendiges Minimum reduziert.

Der DynaNetz-Ansatz ist dabei mehrfach innovativ. Er bietet eine individualisierte Mobilität und nutzt ein innovatives, dynamisches Routingsystem. Liniennetze werden flexibel und in Echtzeit an die aktuelle Nachfrage angepasst. Eine Echtzeitplanung auf der Basis vorhandener Auskunftsdaten ermöglicht eine optimierte Auslastung.

\section{PROJEKTWEBSEITE}

www.technik-zum-menschenbringen.de/projekte/dynanetz

KONTAKT

philip.michalk@th-wildau.de 


\section{Baltic ForBio: Accelerating production of forest bioenergy in the Baltic Sea Region}

Prof. Dr. Jens Wollenweber

PROJEKTMITARBEITER

KOOPERATIONSPARTNER

ASSOZIIERTE PARTNER:

PROJEKTVOLUMEN

MITTELGEBER

LAUFZEIT
Dipl.-Forstw. Mareike Schultze, Dipl.-Wirtsch.-Ing. (FH) Anne-Katrin Osdoba

Schweden: Swedish University of Agricultural Sciences (Lead Partner), The Forestry Research Institute of Sweden, Swedish Forest Agency

Estland: Foundation Private Forest Centre, Tartu Regional Energy Agency

Finnland: Natural Resources Institute Finland, Regional Council Central Finland

Deutschland: Forstdienstleistungen Ueckermann, Schloßgut Altlandsberg GmbH

Lettland: Latvian Rural Advisory and Training Centre - Forest Advisory Service Centre, Latvian State Forest Research Institute SILAVA

Litauen: Kaunas Forestry and Environmental Engineering University of Applied Science

Finnland: The Central Union of Agricultural Producers and Forest Owners, The Ministry of Agriculture and Forestry

Estland: Estonian forest owners association

Deutschland: Ministerium für Ländliche Entwicklung, Umwelt und Landwirtschaft des Landes Brandenburg

Gesamtvolumen: $2.550 .100 €$, Anteil TH Wildau: $367.077 €$

EU | Interreg | Baltic Sea Region

$10 / 2017-09 / 2020$
Biomasse aus Wäldern ist eine wichtige Quelle erneuerbarer Energie im Ostseeraum. Baltic ForBio zielt darauf ab, die Produktion und Nutzung forstlicher Biomasse in den Ostseeanrainerstaaten unter besonderer Beachtung von Nachhaltigkeitskriterien zu sichern und zu steigern.

Im Fokus stehen dabei Waldrestholz aus der Holzernte und Schwachholz aus der Waldpflege - Holzmengen, die sowohl aus ökonomischen als auch aus ökologischen Gründen bisher vielfach ungenutzt bleiben.

Im Dialog mit den wichtigsten Anwender- und Interessengruppen werden kosteneffiziente, stofflich nachhaltige und umweltverträgliche Methoden zur Gewinnung und Nutzung dieser Ressourcen (weiter-) entwickelt und verstärkt verbreitet.

Projektergebnisse des Gesamtprojektes mit Relevanz in Brandenburg sind:
- Identifikation von Erfolgsfaktoren, Entwicklung innovativer Geschäftsmodelle und Handreichungen für den Betrieb kleiner, lokaler Biomasseanlagen und kleinmaßstäblicher Biomasseprojekte im ländlichen Raum

- Tool zur Unterstützung von Entscheidungen, Handbücher, Leitfäden zur Bereitstellung von Waldrestholz und Schwachholz

- Demonstrationsflächen für die nachhaltige Gewinnung von Biomasse aus der Jungbestandspflege

- Trainingsprogramme zur Planung von Holzerntemaßnahmen, zur Biomassegewinnung und zur zugehörigen Logistik sowie zur kosteneffizienten und nachhaltigen Gewinnung von Waldrestholz und Schwachholz aus der Jungbestandspflege
Für die TH Wildau (Forschungsgruppe Verkehrslogistik) stehen die logistischen Gestaltungsmöglichkeiten von der Waldpflege über die Bereitstellungsprozesse bis hin zur Nutzung von Energieholzprodukten in dezentralen Biomasseanlagen im Vordergrund.

Der Fokus in Brandenburg liegt auf einer verstärkten Integration von Nachhaltigkeitskriterien in forstliche Maßnahmenentscheidungen und auf der dezentralen Produktion von Bioenergie im ländlichen Raum. Baltic ForBio wird durch die Entwicklung neuer Erntemethoden zur Lösung bestehender Probleme in der Waldpflege beitragen. Die Vereinbarkeit von Energieholznutzung und Ökosystemleistungen sowie Schutzfunktionen des Waldes sollen verbessert und Übernutzungen vermieden werden. Die Entwicklung lokaler Energiekonzepte und innovativer Geschäftsmodelle für kleine Bioenergieanlagen in 
Brandenburg und die damit verbundene regionale Wertschöpfung durch die Eigenversorgung von Kommunen mit Bioenergie sollen vorangetrieben werden.

\section{PROJEKTWEBSEITE}

WWW.projects.interreg-baltic. eu/projects/baltic-forbio-127. html\#information

\section{KONTAKT}

mareike.schultze@th-wildau.de

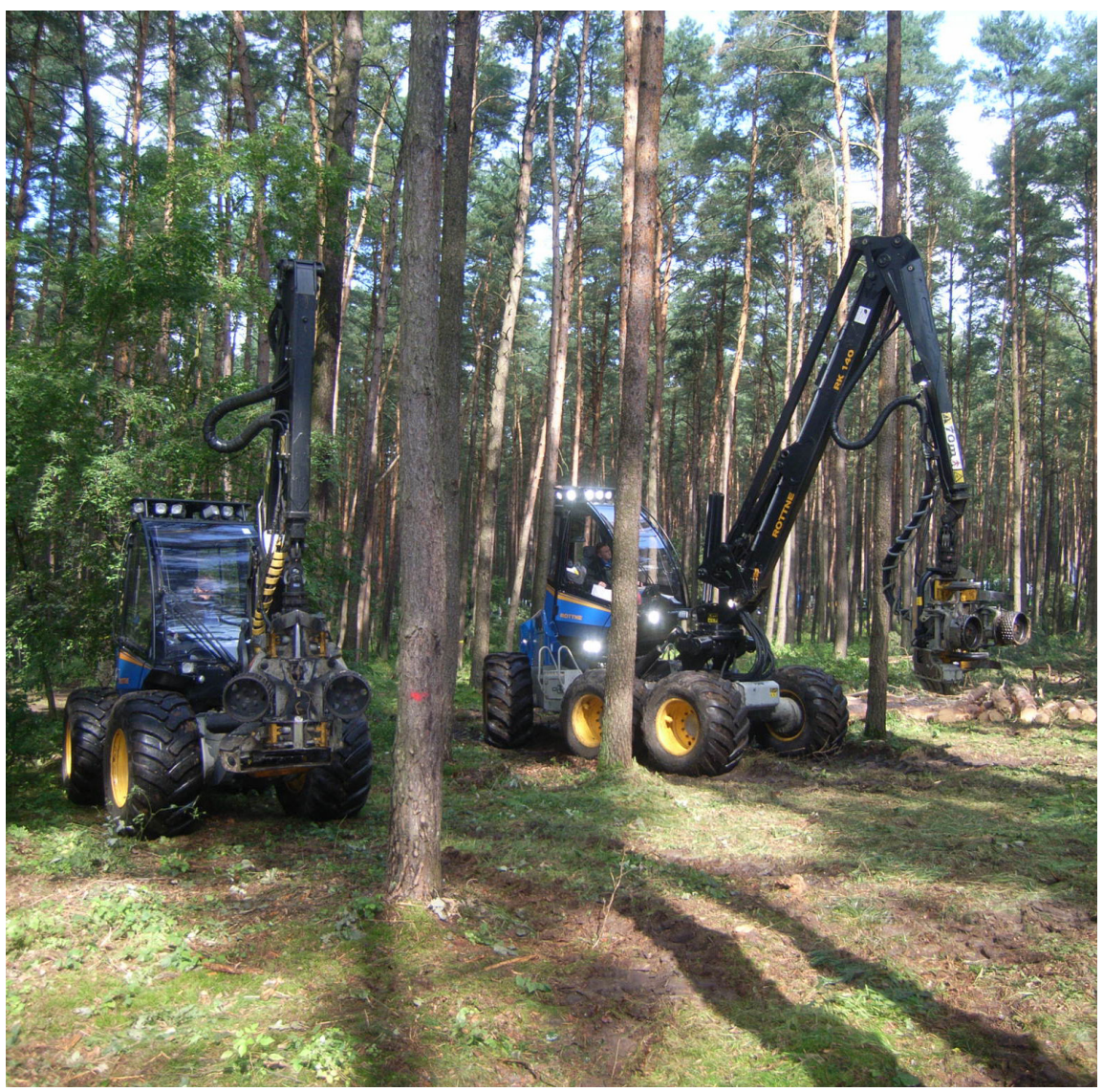

Abb. 1) Harvesterdemonstration

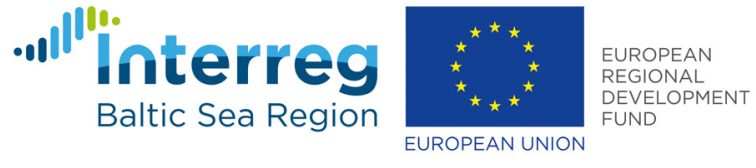

\section{Baltic ForBio}




\section{TABELLARISCHE ÜBERSICHT LAUFENDER PROJEKTE}

Intelligenter Kühlschrank: Versuchsaufbau zur Untersuchung der Anwendbarkeit von RFID Technologie für mit Nahrung befüllte Kunststoffschalen in Kühleinheiten

\begin{tabular}{|c|c|}
\hline Projektleiter & Prof. Dr.-Ing. Frank Gillert \\
\hline Mitarbeiter & Sebastian Krautz \\
\hline Kooperationspartner & Wirtschaft \\
\hline Mittelgeber & My Minute Meal GmbH \\
\hline Laufzeit & $04 / 2017$ \\
\hline
\end{tabular}

IwU KMU: Ausbau von Industrie-4.0-Aktivitäten zur wissenschaftlichen Unterstützung von regionalen KMU

\begin{tabular}{l|l|}
\hline $\begin{array}{l}\text { Projektleiter } \\
\text { Mittelgeber }\end{array}$ & Prof. Dr.-Ing. Frank Gillert \\
\hline $\begin{array}{l}\text { Projektvolumen } \\
\text { Land Brandenburg I MWFK I EFRE | InfraFEI }\end{array}$ \\
\hline Laufzeit & $02 / 2016-06 / 2017$ \\
\hline $\begin{array}{l}\text { Das Projekt dient der Erweiterung der Intralogistikumgebung der Hochschule hin zu einer geschlossenen Industrie } \\
\text { 4.0-Umgebung. Damit soll angewandte Forschung zu Themen rund um Industrie 4.0 in Kooperation mit KMU gefördert } \\
\text { werden. Dabei werden moderne Sensoriken und automatische Identifikationstechnologien genutzt, um Prozesse zu au- } \\
\text { tomatisieren und autonomen Betrieb zu ermöglichen. }\end{array}$
\end{tabular}

WiSima: Wirtschaftlichkeit von Sicherheitsmaßnahmen im öffentlichen Personenverkehr

\begin{tabular}{|c|c|}
\hline Projektleiter & Prof. Dr.-Ing. Frank Gillert \\
\hline Mitarbeiter & Jan Seitz \\
\hline Mittelgeber & Bund | BMBF | Forschung für die zivile Sicherheit \\
\hline Projektvolumen & $355.569 €$ \\
\hline Laufzeit & $07 / 2016-06 / 2019$ \\
\hline \multicolumn{2}{|c|}{$\begin{array}{l}\text { Das Projekt adressiert die Konzeption und Durchführung eines Security Impact Assessments (SIA) als Werkzeug zur Be- } \\
\text { wertung der Wirtschaftlichkeit von Sicherheitsmaßnahmen im öffentlichen Personenverkehr (ÖPV). Neben der Erhöhung } \\
\text { der Sicherheit zielt der Einsatz dieses Werkzeugs auf die Verbesserung der wirtschaftlichen Aspekte ab, indem es die Be- } \\
\text { wertung von Effektivität und Effizienz bestehender Sicherheitsmaßnahmen ermöglicht und so Fragen nach der Ökonomie } \\
\text { der Sicherheit beantworten kann. }\end{array}$} \\
\hline
\end{tabular}


ELLI: Elektroantrieb für Leichtflugzeug Innovation

\begin{tabular}{|c|c|}
\hline Projektleiter & Prof. Dr.-Ing. Wolfgang Rüther-Kindel \\
\hline Mittelgeber & Bund | BMWi \\
\hline Projektvolumen & $499.600 €$ \\
\hline Laufzeit & $01 / 2016-12 / 2017$ \\
\hline
\end{tabular}

Ziel des Vorhabens ELLI ist die Umsetzung eines Elktroantriebssystems für eine neue Generation eines hochinnovativen Leichtflugzeugs.

\section{SeSL: Studie zur elektromagnetischen Schirmung von unbemannten Luftfahrtsystemen}

\begin{tabular}{l|l} 
Projektleiter $\quad$ Prof. Dr.-Ing. Wolfgang Rüther-Kindel
\end{tabular}
Mitarbeiter
Dr. Sven Angermann

Kooperationspartner navtec Microwave $\mathrm{GmbH}$

Mittelgeber FuE | BIG-Transfer klein

\begin{tabular}{l|l} 
Projektvolumen & $3.000 €$
\end{tabular}

Laufzeit 04/2017-09/2017

In dem beantragten Vorhaben SeSL soll untersucht werden, ob es technisch möglich ist, ein flugfähiges unbemanntes Luftfahrtsystem derart gegen elektromagnetische Störungen zu Schirmen, dass Flüge in einer derartigen Umgebung zuverlässig durchgeführt werden können.

\section{Baltic ForBio: Accelerating Production of Forest Bioenergy in Baltic Sea Region}

Projektleiter

Prof. Dr. Jens Wollenweber (Prof. Dr.-Ing. Herbert Sonntag)

Mitarbeiter

Mareike Schultze, Anne-Katrin Osdoba, Mike Lange

Kooperationspartner

13 Partner aus Schweden, Estland, Finnland, Deutschland, Lettland, Litauen

Mittelgeber

EU I Interreg | Baltic Sea Region

Projektvolumen

$367.077 €$

Laufzeit

$10 / 2017-09 / 2020$

Das Projekt Baltic ForBio leistet einen Beitrag zur nachhaltigen Produktion und Nutzung von Waldenergieholz im Ostseeraum. Es werden kosteneffiziente und nachhaltige Methoden zur Nutzung von Waldrestholz und Schwachholz im Dialog mit den wichtigsten Anwendergruppen entwickelt bzw. weiterentwickelt und verstärkt verbreitet. 
Die Liberalisierung des Busmarktes, hat vor allem in Deutschland aber auch in den Nachbarländern zu einem signifikanten Anstieg der Fernbusverkehre geführt. Die meist innerstädtisch gelegenen Busterminals können aber baulich kaum oder nur in sehr begrenztem Maße erweitert werden. Eine Lösung bietet die optimierte Nutzung der vorhandenen Ressourcen um mehr Verkehre ohne bauliche Veränderung abwickeln zu können. DISBUS entwickelt dazu einen IT-basierten Prototyp für die Optimierung von Betriebsabläufen in Fernbusterminals, einschließlich der Schnittstellen zu Fernbusbetreibern und zu Fahrgastinformationssystemen. Das Projekt zielt auf eine Markteinführung des Produktes, nach Abschluss der Forschungsphase ab.

\section{DynaNetz: Dynamisches, individualisiertes Angebotsnetz im Öffentlichen Personennahverkehr}

\begin{tabular}{l|l} 
Projektleiter $\quad$ Prof. Dr. Jens Wollenweber (Prof. Dr.-Ing. Herbert Sonntag)
\end{tabular}

Mitarbeiter Philip Michalk

Kooperationspartner IVU AG, VBB, Verkehrsbetriebe Oberhavel, Door2Door

Mittelgeber Bund I BMBF

\begin{tabular}{l|l} 
Projektvolumen $\quad 118.483 €$
\end{tabular}

Laufzeit $\quad 12 / 2017-11 / 2018$

Heutige ÖPNV-Angebotsnetze sind in aller Regel statisch: Die Standorte von Haltestellen sind ebenso wie die Routenverläufe der Fahrzeuge und Abfahrtszeiten langfristig geplant und vorgegeben. Dies führt zu einer unbefriedigenden Angebotssituation. Hier setzt DynaNetz an: Im Rahmen des Projektes wird ein Konzept entwickelt, das die dynamische Planung von Angebotsnetzen erlaubt. Basierend auf Anfragedaten wird ein ÖPNV-Angebotsnetz in Echtzeit angepasst und mit elektr. Kleinbussen bedient.

Mini-Harvester - Entwicklung eines vollmechanisierten Ernteverfahrens zur Pflege von Jungbeständen

\begin{tabular}{l|l} 
Projektleiter Prof. Dr. Jens Wollenweber (Prof. Dr.-Ing. Herbert Sonntag)
\end{tabular}

Mitarbeiter Mareike Schultze, Mike Lange, Anne-Katrin Osdoba

Tyroller Hydraulik Herzberg GmbH, Möhle und Braker Datentechnik GbR,

Kooperationspartner Forstdienstleistungen Ueckermann, Landesbetrieb Forst Brandenburg, Kuratorium für Waldarbeit und Forsttechnik (KWF)

Mittelgeber

Bund | BMEL | FNR

Projektvolumen $198.460 €$

Laufzeit

10/2015-11/2018

Ziel sind Entwicklung, Test und Bewertung eines funktionsfähigen vollmechanisierten Verfahrens für die Pflege junger Waldbestände mit Kleintechnik. Damit wird eine Verfahrenslücke zur Anwendung im kleinstrukturierten Wald und auf Waldumbauflächen geschlossen. Das wissenschaftliche Hauptziel ist die Erarbeitung von Grunddaten zur Bewertung eines spezifischen Pflege- und Bereitstellungsverfahrens als Beitrag im Bereich der forsttechnischen Forschung. 
Projektleiter

Mitarbeiter

Kooperationspartner

Mittelgeber

Projektvolumen

Laufzeit

Projekthomepage
Prof. Dr. Jens Wollenweber (Prof. Dr.-Ing. Herbert Sonntag)

Philip Michalk

15 Partner aus Schweden, Dänemark, Norwegen und Deutschland

EU | Interreg | Baltic Sea Region

$293.605 €$

$05 / 2016-05 / 2019$

www.scandria-corridor.eu/index.php/en/projects/scandria2-act

Scandria II Act ist ein gemeinsames Projekt von Regionen und Forschungseinrichtungen entlang des Kernnetz-Korridors von Skandinavien an die Adria (Scanmed). Es unterstützt regionale Entwicklungsmaßnahmen im Rahmen der Europäischen Transportpolitik. Schwerpunkte des Projekts liegen in den Bereichen „Alternative Antriebe“, "Multimodaler Transport“ und „Trans-Governance“.

\section{SEEN-KV: Simulation des Einsatzes Elektrischer Nutzfahrzeuge im KV}

Projektleiter

Prof. Dr. Jens Wollenweber (Prof. Dr.-Ing. Herbert Sonntag)

Mitarbeiter

Martin Jung, Philip Michalk

Kooperationspartner

LUB Consult, EBO-Sächsische Häfen Oberelbe, Emons

Mittelgeber

Bund | BMVI

Projektvolumen

$141.936 €$

Laufzeit

$10 / 2016-09 / 2018$

Entwicklung eines Simulationsansatzes zur Erforschung und Erprobung von vollelektrischen Fahrzeugflotten im Umschlag und im Nachlauf des kombinierten Verkehr.

\section{VARMA - Value added by optimal wood raw material allocation and processing}

Projektleiter

Mitarbeiter

Kooperationspartner

Mittelgeber

Projektvolumen

Laufzeit

Projekthomepage
Prof. Dr. Jens Wollenweber (Prof. Dr.-Ing. Herbert Sonntag)

Anne-Katrin Osdoba, Mareike Schultze, Mike Lange

12 Partner aus Finnland, Deutschland, Frankreich, Schottland

Bund | BMEL | FNR | ERA-Net WoodWisdomNet+

$122.270 €$

07/2014-06/2017

www.varma-eu.com/

Die Forst- und Holzwirtschaft steht vor der Herausforderung, dem stetig wachsenden Bedarf an Rohstoffen für eine breite Fülle an Nutzungspfaden zu begegnen und die Akteure wettbewerbsfähig aufzustellen. Dies ist auch mit der Aufgabe verbunden, Holzrohstoffe waldseitig versorgungssicher zu verteilen. VARMA greift die entstehenden Herausforderungen auf und hat das Ziel, z.T. grundlegend neue Modelle und Wertschöpfungsketten zu konzipieren. 
Networking DePol - Networking der wirtschaftsfördernden Einrichtungen im deutsch-polnischen Raum

Projektleiter

Prof. Dr. Jens Wollenweber (Prof. Dr.-Ing. Herbert Sonntag)

Mitarbeiter

Philip Michalk

Kooperationspartner

Div. Partner aus Polen und Deutschland

Mittelgeber

EU | Interreg IVB

Projektvolumen

$30.000 €$

Laufzeit

$12 / 2017-12 / 2018$

Die TH Wildau entwickelt Methoden zur Beurteilung der Erfolgsaussichten von Logistikwirtschafltichen Unternehmen im Grenzraum zwischen Brandenburg und Polen. 


\section{TABELLARISCHE ÜBERSICHT PUBLIKATIONEN}

Caimi G, Kroon L, Liebchen C (2017)

Goerigk M, Liebchen C (2017)

Ichkitidze Y, Sarygulov A, Ungvári L (2017)

Liebchen C (2017)

Melzer K-M, Siegmann J, Heinrich I (2017)

Michalk P, Schmidt C, Jung M (2017)
On the Specific Application Fields of Various Models for Railway Timetable Optimization. In: Tomii N, Hansen I, Rodriguez J, Pellegrini P, et al. (eds) RailLille 2017 - Proceedings. IFSTTAR \& IAROR, Marne-la-Vallée Cedex, France, ISBN: 978-2-85782-723-8, pp 280-301

An Improved Algorithm for the Periodic Timetabling Problem. 12. In: D'Angelo G, Dollevoet T (eds) 17th Workshop on Algorithmic Approaches for Transportation Modelling, Optimization, and Systems (ATMOS 2017). OASIcs - OpenAccess Series in Informatics. Schloss Dagstuhl-Leibniz-Zentrum fuer Informatik, Dagstuhl, Germany, ISBN: 978-3-95977042-2, 12:1-12:14. doi: 10.4230/OASIcs.ATMOS.2017.12

Potential for Enhancing Traffic Safety on Highways of Russia. Transportation Research Procedia 20:242-246. doi: 10.1016/j.trpro.2017.01.058

Quo vadis Taktfahrplanoptimierung im Eisenbahnverkehr? Eisenbahntechnische Rundschau 66(1+2):31-35

Planbarer Umgang mit der außerplanmäßigen Instandhaltung von Eisenbahnfahrzeugen - Paradoxon oder Chance? Algorithmus zur Herleitung eines vorhersagbaren Schadindexes unter wirtschaftlicher Berücksichtigung betrieblicher Ressourcen. Eisenbahntechnische Rundschau 66(12):55-63

Prozessoptimierung als Mittel der Kapazitätsmaximierung von Fernbusterminals. In: Barton T, Müller C, Seel C (eds) Geschäftsprozesse: Von der Modellierung zur Implementierung. Angewandte Wirtschaftsinformatik. Springer Fachmedien Wiesbaden, Wiesbaden, ISBN: 978-3-658-17297-8, pp 211-219. doi: 10.1007/978-3-658-17297-8_11

Übungsbuch Logistik-Entscheidungen. De Gruyter, Berlin und Boston. ISBN: 978-3-11-044033-1 
Management und Recht Management and Law 


\title{
Coaching Pilot für den Berufseinstieg von Studierenden im Land Brandenburg
}

\author{
Prof. Dr. Dana Mietzner
}

\author{
PROJEKTMITARBEITER(INNEN) Markus Lahr \\ KOOPERATIONSPARTNER Klein- und mittelständische Unternehmen (KMU) / Organisationen \\ PROJEKTVOLUMEN \\ $290.660 €$ \\ MITTELGEBER \\ Land Brandenburg | MWFK | ESF \\ LAUFZEIT \\ 01/2016-12/2018
}

Der Berufseinstieg unmittelbar nach dem Studium im Themenfeld Innovationsmanagement stellt für viele Studierende eine Herausforderung dar. Gerade in kleinen und mittleren Unternehmen müssen frühzeitig vielfältige Aufgaben bewältigt und Eigenverantwortung übernommen werden, die mit dem klassischen Innovationsmanagement wenig zu tun haben. Auch deshalb erscheint vielen Absolventen der Einstieg in ein Traineeprogramm eines großen Unternehmens oder ein klar definierter Karrierepfad in der Großindustrie oftmals attraktiver. Andererseits und insbesondere vor dem Hintergrund des Fachkräftemangels sehen sich Absolventen aber auch einem Arbeitsmarkt gegenüber, der viele Möglichkeiten seitens der Brandenburger Unternehmen bietet.

Das Projekt beinhaltet daher die Konzipierung, Umsetzung und Evaluierung eines neuen Coaching-Modells, um den Berufseinstieg von Studierenden zu unterstützen. Fach- und Metho-

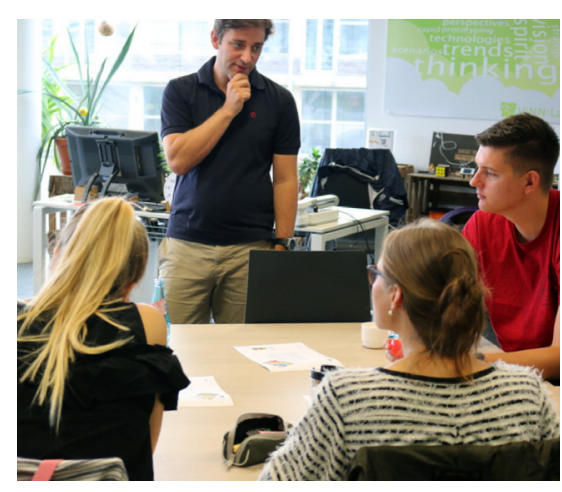

Abb. 1) Idea-Generation im Innovation Camp denwissen sowie andere Vorkenntnisse und Erfahrungen der Teilnehmer/ -innen werden beim Coaching explizit berücksichtigt und an die Persönlichkeitsprofile angepasst. Parallel unterstützt das Projekt Brandenburger Unternehmen, in Kontakt mit zukünftigen Absolventen zu kommen, indem Studierende im Rahmen von Innovation Camps Konzepte zur Lösung spezifischer Innovationsprobleme entwickeln.

Damit soll den spezifischen Bedarfen von kleinen und mittleren Unternehmen ebenso entsprochen werden wie den Ansprüchen einzelner Studierender. Somit kann die Hochschule einem zunehmend heterogenen Studierendenkreis mit unterschiedlichsten Erfahrungs-, Ausbildungs- und Herkunftshintergründen besser gerecht werden.

So wurden im Rahmen des Innovation Camp 2017 gemeinsam mit dem Rundfunk Berlin Brandenburg neue Konzepte für das Fernsehen der Zukunft entwickelt. Im Mittelpunkt standen dabei zukunftsweisende Technologien wie VR und AR, aber auch immersives TV und die Nutzbarmachung komplexer Daten für lineares und nicht lineares Fernsehen. Mit Hilfe der in den Coachings vermittelten Skillsets zu den Themenkomplexen Konfliktmanagement, Kommunikationstheorie und Graphic Recording wurden vier komplexe Konzepte für des Fernsehen der Zukunft entwickelt und dem rbb präsentiert.

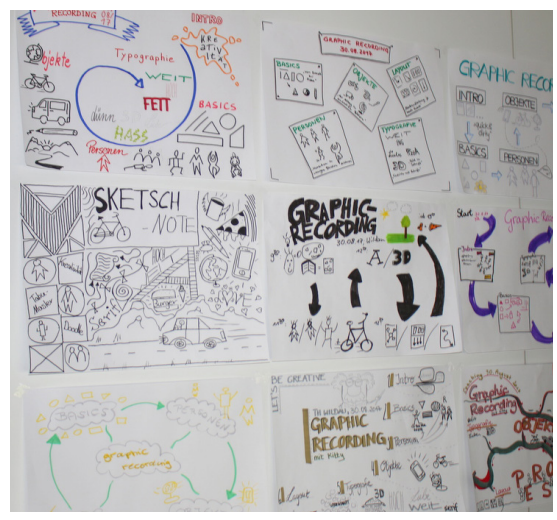

Abb. 2) Coaching Graphic Recording

PROJEKTWEBSEITE

www.th-wildau.de/forschungtransfer/innovations-undregionalforschung/projekte/ coachingpilot

\section{KONTAKT}

dana.mietzner@th-wildau.de 
Gefördert durch das Ministerium für Wissenschaft, Forschung und Kultur aus Mitteln des Europäischen Sozialfonds und des Landes Brandenburg.

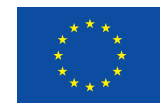

EUROPÄISCHE UNION Europäischer Sozialfonds

Investition in Ihre Zukunft!

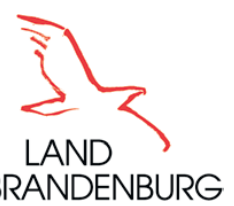

Ministerium für Wissenschaft, Forschung und Kultur 


\title{
Panel Bürgerwissenschaft an der Technischen Hochschule Wildau
}

\author{
Prof. Dr. Dana Mietzner
}

PROJEKTMITARBEITER(INNEN) Dr. Frank Hartmann, M.A. Eva Ismer und Dipl.Ing.-agr. Marion Glowik

PROJEKTVOLUMEN

MITTELGEBER

LAUFZEIT
$60.000 €$

TH Wildau | Zielvereinbarung

$10 / 2016-12 / 2018$
Das Innovationsmanagement in der unternehmerischen Praxis hat sich in den vergangenen Jahren von „Open Innovation“ (Öffnung von Innovationsprozessen gegenüber Partnern und externe Verwertung von F\&EErgebnissen und Innovationen) über „Open Local Innovation“ (Öffnung von Unternehmen für die Zusammenarbeit mit kommunalen Verwaltungen) bis hin zu „Open Collaborative Innovation“ (Einbezug von Bürgern in Innovationsprozesse) und „Free Innovation (Bürger als Innovatoren) entwickelt. Im Wissenschaftsbereich zeichnet sich eine ähnliche Entwicklung ab. Wissenschaft beginnt nun, sich unter dem Schlagwort "Bürgerwissenschaft" bzw. "Citizen Science“ verstärkt auch Laien und zivilgesellschaftlich organisierten Bürgerinnen und Bürgern zu öffnen. Für Hochschulen mit ihren spezifischen Funktionen und Ressourcen im Wissenschaftssystem ergeben sich hiermit neue Herausforderungen und Chancen, die weiter auszuloten sind.

Vor diesem Hintergrund baut die Forschungsgruppe Innovations- und Regionalforschung ein Panel Bürgerwissenschaft auf, das es auch Personen, die hauptberuflich nicht in Wissenschaft und Forschung tätig sind, ermöglicht, sich aktiv an Forschungsprojekten zu beteiligen. Je nach Projekt eröffnen sich unterschiedliche Möglichkeiten der Beteiligung, von der Datenerhebung über das Beobachten bis hin zum gemeinsamen Konzipieren und Umsetzen von Projekten. Gemein- sam ist allen Bürgerwissenschaftsprojekten das Ziel, in Zusammenarbeit mit der Wissenschaft neues Wissen für die Wissenschaft selbst, die Gesellschaft und politische Entscheidungsprozesse zu gewinnen und somit bei der Erarbeitung von Lösungen gesellschaftlich relevanter Problemstellungen mitzuwirken. Dabei kann es sich sowohl um naturwissenschaftlich-technische als auch wirtschafts- und sozialwissenschaftliche Vorhaben handeln.

Interessierte Bürger/ -innen werden beispielsweise aktiv durch

- die Teilnahme an schriftlichen oder mündlichen Befragungen

- das Sammeln und Aufbereiten von Daten

- die Beteiligung an der Ideengewinnung für wissenschaftlich basierte Innovationsvorhaben in KreativWorkshops

- das Testen von Prototypen in Laborumgebungen, um deren Anwendbarkeit zu verbessern

- die Beteiligung an unterschiedlichen Formaten der Zukunftsforschung

Nach einer Phase der wissenschaftlich basierten Konzeption und der Gewinnung interessierter Bürger/ -innen konstituiert sich das Panel an der Technischen Hochschule Wildau Anfang März 2018.

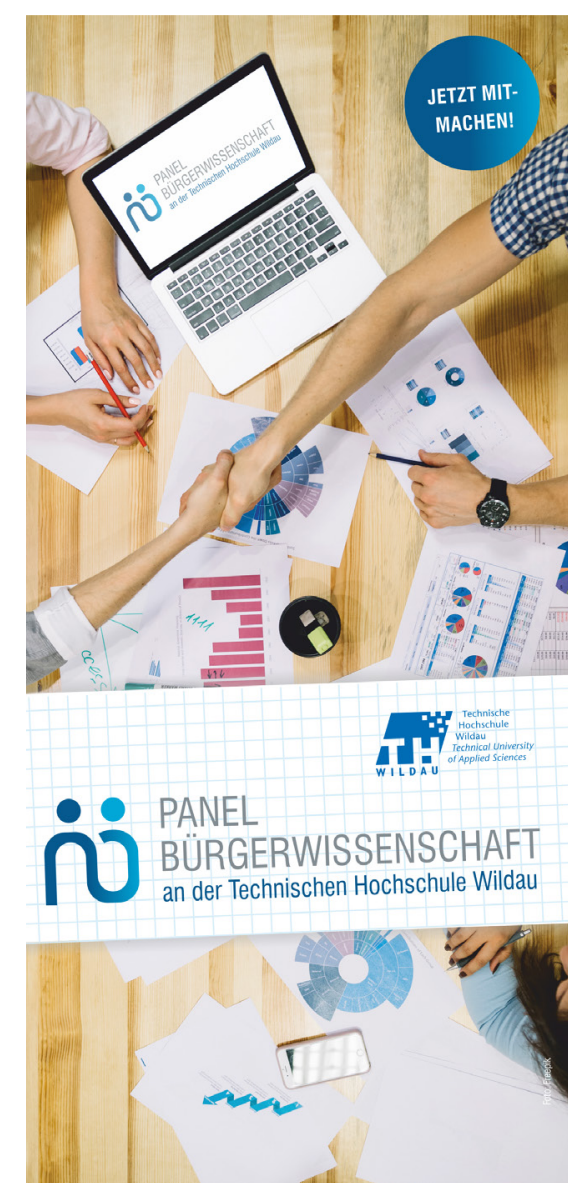

Abb. 1) Flyer zum Projekt

\section{PROJEKTWEBSEITE}

www.th-wildau.de/buergerpanel

\section{KONTAKT}

frank.hartmann@th-wildau.de 


\title{
ViNN:Lab: Erhöhung von Performance \& Impact
}

\author{
Prof. Dr. Dana Mietzner
}

PROJEKTMITARBEITER(INNEN) Eva Ismer, Patrick Grabasch

PROJEKTVOLUMEN

$120.000 €$

MITTELGEBER

TH Wildau | Zielvereinbarung

LAUFZEIT

$10 / 2016-12 / 2018$

Die Infrastruktur und Methoden des ViNN:Lab werden in der Lehre (u. a. im Innovationsmanagement, Technologiemanagement, Logistik und Maschinenbau) eingesetzt und im Rahmen von angewandten Forschungsprojekten von unterschiedlichen Forschungsgruppen, Instituten und Projekten der TH Wildau genutzt. Neben Forschung und Lehre leistet das ViNN:Lab einen Beitrag für den Technologietransfer. In diesem Zusammenhang ist das ViNN:Lab regelmäßig auf regionalen und überregionalen Messen und Ausstellungen (u. a. Lange Nacht der Wissenschaften, Tag der Wissenschaften, Maker Faire Berlin, FabFestival Toulouse, Tag der offenen Bundesregierung sowie der UNESCO-Bildungsmesse).

Das ursprünglich mit dem ViNN:Lab intendierte Konzept, die Förderung von Interdisziplinarität innerhalb und außerhalb der Hochschule und die Unterstützung von offenen (kollaborativen) Innovationsansätzen durch die Bereitstellung von Infrastruktur und Methoden, hat sich um wichtige Themenfelder erweitert. Beispiele dafür sind der wöchentlich stattfindende Open Lab Day (Fab Lab), der nicht nur Studierende und Schüler/ -innen, sondern auch die Zivilgesellschaft, KMU, Institutionen/Organisationen (z. B. Blindenschule Königs Wusterhausen, Berlinische Galerie, Landesmuseum Sachsen-Anhalt, Deutsches Historisches Museum), Start-ups und Vertreter/-innen von Forschungseinrichtungen regelmäßig anzieht.

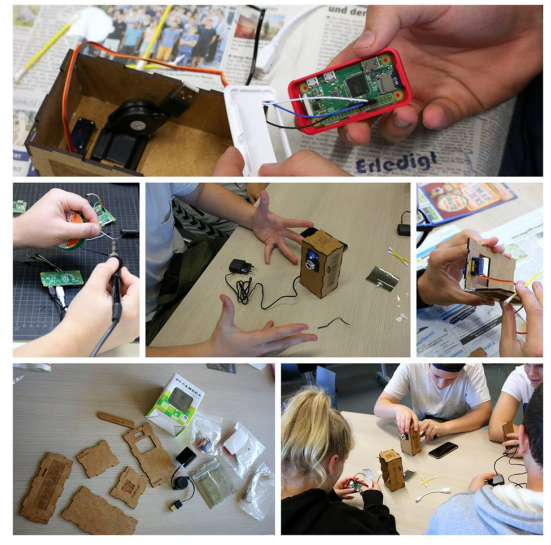

Abb. 1) Workshop „Bau einer Dolpi-Kamera“

Ziel des Vorhabens „Impact \& Performance" ist es, bestehende Potenziale in den Bereichen der Nutzerinteraktion und -reichweite besser zu nutzen und dadurch die hohe regionale und überregionale Ausstrahlkraft des ViNN:Labs, die Integration in die Lehre sowie die Nutzung im Rahmen interdisziplinärer Forschung weiter zu verbessern.

So wurden im Jahr $2017 \mathrm{im}$ Rahmen des Teilprojektes KiVi:Lab 55 Workshops unterschiedlicher Komplexitätsgrade für 787 Schülerinnen und Schüler durchgeführt, um das Interesse an Prototyping-Technologien frühzeitig zu fördern und das kreative Problemlösen zu unterstützen. Des Weiteren konnten Projekte mit dem Naturkundemuseum Bern, dem Deutschen Historischen Museum Berlin und dem Auswärtigen Amt erfolgreich abgeschlossen werden. Darüber hinaus wurde der Austausch von Best-Practice-

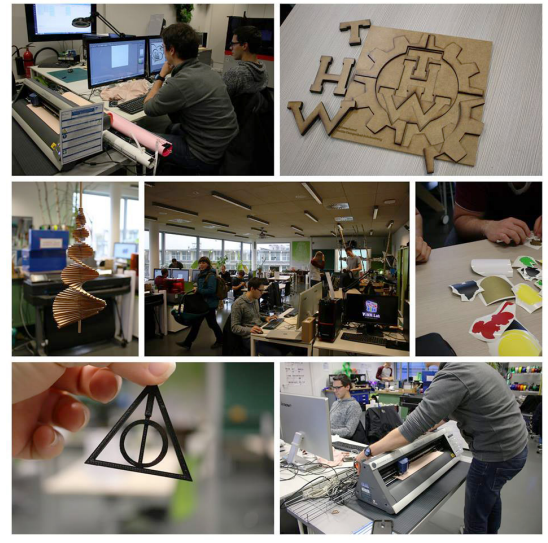

Abb. 2) Open Lab Day

Ansätzen mit nationalen HochschulFablabs angeregt, gemeinsame Workshops wurden konzipiert und umgesetzt sowie regelmäßige Austauschformate etabliert. Des Weiteren wurden die im ViNN:Lab durch die Nutzer realisierten Projekte im Rahmen von systematischen Nutzer-Befragungen erhoben, um eine zielgruppenspezifische Weiterentwicklung von kollaborativen Innovationsansätzen im Rahmen des ViNN:Lab zu unterstützen.

\section{PROJEKTWEBSEITE}

www.th-wildau.de/vinnlab

\section{KONTAKT}

dana.mietzner@th-wildau.de 


\section{ViTeP: Virtuelle interdisziplinäre Team- und Projektarbeit}

Prof. Dr. Dana Mietzner

PROJEKTMITARBEITER(INNEN) Bianca Baumann (FG Innovations- und Regionalforschung)

PROJEKTVOLUMEN

MITTELGEBER

LAUFZEIT
$54.190 €$

TH Wildau | Zielvereinbarung

03/2016-12/2018
Der Zuwachs projektförmiger \& virtueller (Team-) Arbeit, die steigende Bedeutsamkeit des routinierten Umgangs mit digitalen Technologien und Medien sowie die Notwendigkeit ausgeprägter Soft Skills sind nicht nur Treiber neuer Arbeitsformen, sondern auch Strukturgeber für die Kompetenz- und Anforderungsprofile von Arbeitnehmenden der Zukunft. Um Studierende der Fachbereiche Wirtschaft, Informatik, Recht (WIR) und Ingenieur- und Naturwissenschaften (INW) umfassender auf das Arbeiten in einer digitalen Arbeitswelt mit verteilten Teams vorzubereiten, soll das Modul „ViTeP - Virtuelle Team- und Projektarbeit" etabliert werden.

Das als Blended Learning konzipierte ViTeP-Modul unterteilt sich dabei in spezifische "Arbeits- und Lernpakete", die vor allem Grundlagenwissen zum Projektmanagement sowie zu (virtuellen) Teambildungs- und Interaktionsprozessen thematisch fokussieren (vgl. Abb. 1).

Dabei unterstützen sogenannte „ViTePEinheiten" die Auseinandersetzung mit den jeweiligen thematischen Schwerpunkten während der Distanzphasen bzw. virtuellen Selbstlernphasen. So werden Wissensinhalte vermittelt, digitale Tools zur kollaborativen Team- und Projektarbeit genutzt sowie Gelerntes und Angewandtes parallel reflektiert und im Rahmen eines Praxisprojektes als (virtuelle) Gruppenarbeit umgesetzt (vgl. Abb. 2).

In Anlehnung an Deeper-LearningMethoden, die vor allem die Vermittlung von Lerninhalten durch kritisches Denken, Teamarbeit, Problemlösungsmethoden und selbstbestimmtes
Lernen fokussieren, zeichnet sich das Modul ViTeP somit u. a. neben der Verlagerung der Team- und Projektarbeit in virtuelle Räume durch folgende Schwerpunkte aus:

- Digitaler Arbeits- und Teamfahrplan (ViTeP-Plan) zur kontinuierlichen Verbesserung in der Benutzung von digitalen Medien und Technologien

- Peer-Tutorin/Peer-Tutor zur virtuellen Betreuung und Unterstützung digitaler Lernprozesse der Studierenden

- E-Learning-Tagebuch zur Protokollierung und Dokumentation von Erfahrungs- und Lernfortschritten sowie zur Schulung der Selbstreflexion, des Lerntransfers und des Perspektivenwechsels

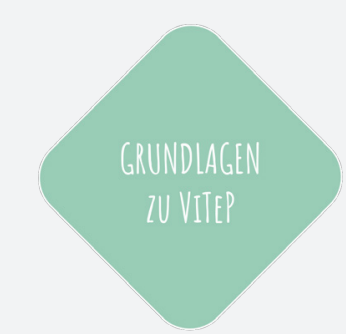

Paket 1 | Einführung in die virtuelle Team- und Projektarbeit

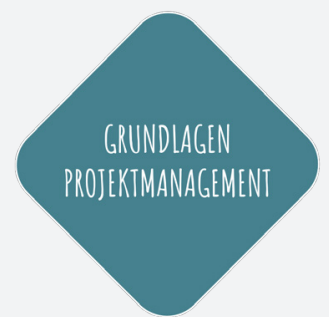

Paket 2 | Methoden \& Frameworks des Projektmanagements

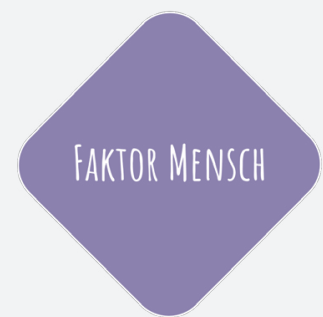

Paket 3 | Prozesse \& Strategien von Teams und Teambuilding

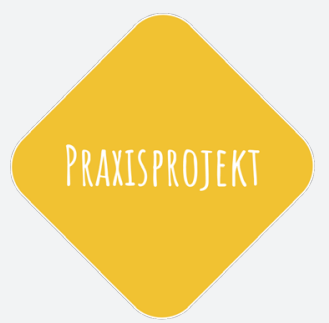

Paket 4 | Anwendung virtueller Projektarbeit im Team 
Nach Beendigung der Pilotierungsphase des ViTeP-Moduls ist darüber hinaus vorgesehen, Transferworkshops für alle interessierten Einzelpersonen und Teams der TH Wildau zu konzipieren und durchzuführen. Somit können nicht nur Lehrkräfte an den Erkenntnissen zu digitalen Lehransätzen im Hochschulkontext sowie zur virtuellen Projektarbeit partizipieren, sondern auch Forschungs- und Arbeitsgruppen der TH Wildau.

\section{KONTAKT}

bianca.baumann@th-wildau.de

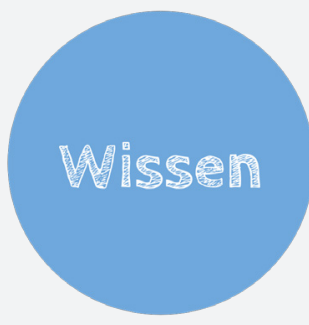

Wissenseinheiten zu Projektmanagement und virtuellen Teams

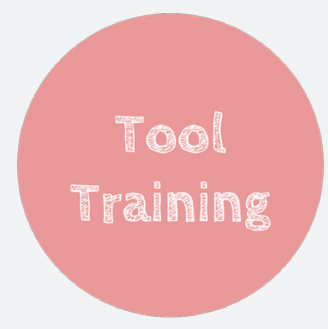

Trainingseinheiten zu Tools kollaborativer Projektarbeit

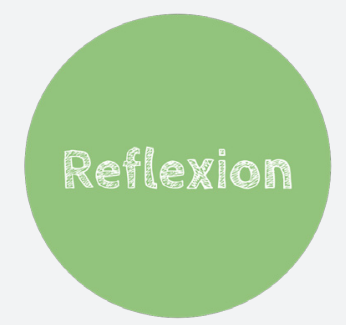

Reflexionen zu Wissens- und Trainingseinheiten
Praxis 9)

Anwendung der Wissens- und Trainingseinheiten 


\section{TABELLARISCHE ÜBERSICHT LAUFENDER PROJEKTE}

\section{Marketing Deceptions in German Practices}

Projektleiter

Prof. Dr. iur. Katrin Blasek, Prof. Dr. rer. pol. Sandra Haas

Kooperationspartner

Universität Oldenburg: Prof. Dr. Thomas Raabe

Laufzeit

01/2014-12/2017

The interdisciplinary project „Marketing deceptions in German Practices" focuses on: a) Providing a systematic review of pubilshed cases on false marketing practices in Germany by analyzing cases in terms of published users, using types as well as in terms of violations against § 5 UCA or against the even more undesirable blacklisted per se bans that were introduced in 2008 to impelent EU regulations. b) Analyzing how marketing deceptions are presented within the German media ladscape.

\section{ECMT+: Entrepreneurship and Communication in Multicultural Teams}

\begin{tabular}{|c|c|}
\hline Projektleiter & Dr. Gregory Bond \\
\hline Mittelgeber & EU | Erasmus+ | Key Action 2 Strategic Partnerships \\
\hline Projektvolumen & $56.000 €$ \\
\hline Laufzeit & $09 / 2016-08 / 2019$ \\
\hline
\end{tabular}

Diese Projekt ist eine Kooperation von sieben europäischen Hochschulen. Das Projket hat drei Säulen: Zum ersten werden Studierende bei internationalen Workshops unternehmerisches Denken und interkulturelle Arbeit praktizieren. In einem begleitenden Forschungsprojekt werden Lehrmethoden für interkulturelles Unternehmertum entwickelt und getestet. Die dritte Komponente ist die Etablierung einer „Community of Practice“ unter Lehrenden.

\section{B-Watch - Entwicklung eines sensorbasierten Bewegungsanalysesystems}

Projektleiter Prof. Dr. phil. Bertil Haack, Prof. Dr. rer. nat. Thomas Goldmann

Kooperationspartner Charité Berlin, DreiF Informationstechnologie GmbH, SATIS INFORMATION GmbH

Mittelgeber Bund | BMWi | ZIM

\begin{tabular}{l|l} 
Projektvolumen $\quad 174.220 €$
\end{tabular}

Laufzeit $\quad$ 07/2014-06/2017

Im Rahmen der sensormotorischen Behandlung von Patienten mit Parkinson-Syndromen, Schlaganfall und anderen neurologischen Erkrankungen ist es erforderlich, Bewegungen präzise, objektiv und ohne hohen technischen bzw. zeitlichen Aufwand zu untersuchen. Beabsichtigt ist die Entwicklung eines sensorbasierten Systems zur Bewegungsanalyse im Prototypen insbesondere für die klinische Anwendung bei Parkinson- und Schlaganfall-Patienten, jedoch mit der Erweiterungsoption auf weitere Anwendungsbereiche. 
Projektleiter

Mitarbeiter

Kooperationspartner

Mittelgeber

Projektvolumen

Laufzeit

Prof. Dr. phil. Bertil Haack

\section{Fr. Anne Herrmann, Hr. Dr. Alfred Krause}

Brandenburgische Kommunalakademie (BKA)

Brandenburgische Kommunalakademie

$1.755 .000 €$

09/2009-09/2017

Es ist geplant, den Studiengang "Kommunales Verwaltungsmanagement und Recht" seitens der TH Wildau in Kooperation mit der Brandenburgischen Kommunalakademie in Potsdam zu konzipieren und umzusetzen. Dieser Studiengang wird als Bachelor-Studiengang im Fachbereich Wirtschaft, Verwaltung und Recht der TH Wildau integriert sein. Er wird unter Leitung und Führung der TH Wildau sowohl an der TH Wildau, als auch bei der Brandenburgischen Kommunalakademie in Potsdam in enger Anlehnung an die Praxis und die dortige Ausbildung zum Verwaltungsfachwirt (analog gehobener Dienst) durchgeführt. Die Semester 1-3 des Studienganges werden an der BKA, die Semester 4-6 an der TH Wildau realisiert. Gemäß Plan werden KVR-Studierende zum SomSem 2011 ihr Studium an der TH Wildau aufnehmen.

\section{STAY IN TOUCH: MINTalent Relationship Management an der TH Wildau}

\begin{tabular}{l|l}
\hline Projektleiter & Prof. Dr. rer. pol. Sandra Haas \\
\hline Mittelgeber & Land Brandenburg I MWFK | ESF \\
\hline Projektvolumen & $250.616 €$ \\
\hline Laufzeit & $01 / 2016-12 / 2018$ \\
\hline
\end{tabular}

Unter Anwendung des betrieblichen Talent Relationship Management Ansatzes werden versteckte MINT-Talente an den Oberstufenzentren (= sudierfähige beruflich Qualifizierte, die sich ihrer Studierfähigkeit nicht bewusst sind) als akademischer MINT-Nachwuchs erschlossen. Ein besonderes Augenmerk liegt dabei in der Entwicklung von versteckten Talenten aus bildungsfernen Haushalten und/oder mit Migrationshintergrund.

Integra 2 - Integration von Flüchtlingen ins Fachstudium

\begin{tabular}{l|l}
$\begin{array}{l}\text { Projektleiter } \\
\text { Mittelgeber }\end{array}$ & Dr. rer. nat. Katrin Kuhls \\
\hline $\begin{array}{l}\text { PAAD } \\
\text { Projektvolumen }\end{array}$ & $174.000 €$ \\
\hline Laufzeit & $01 / 2017-12 / 2018$ \\
\hline $\begin{array}{l}\text { Integra } 2 \text { fördert Studienvorbereitungskurse für Geflüchtete im Rahmen der Welcome@TH-Wildau-Initiative (Ein- und } \\
\text { Zweijahresprogramme). Diese Kurse ermöglichen eine komprimierte Sprachausbildung von A1 bis C1 mit dem Ziel DSH-2 } \\
\text { Prüfung (Voraussetzung für die Zulassung zum Studium). Durch den begleitenden Fachunterricht werden die Kursteilneh- } \\
\text { mer fachlich und fachsprachlich auf ein technisches, naturwissenschaftliches oder wirtschaftliches Studium in Deutsch- } \\
\text { land vorbereitet. }\end{array}$
\end{tabular}


Welcome Team 2 - Studierende engagieren sich für Flüchtlinge

\begin{tabular}{|c|c|}
\hline Projektleiter & Dr. rer. nat. Katrin Kuhls \\
\hline Mittelgeber & DAAD \\
\hline Projektvolumen & $54.000 €$ \\
\hline Laufzeit & 01/2017-12/2018 \\
\hline
\end{tabular}

Die TH Wildau bietet verschiedene Maßnahmen zur Flüchtlingsintegration an. Diese werden unter der Leitung des Welcome Centers und in enger Kooperation mit dem International Office und dem TH Wildau College umgesetzt. Dazu werden mehrere über dieses Projekt finanzierte studentische Hilfskräfte eingesetzt. Die Aktivitäten beziehen sich u.a. auf die Unterstützung bei der Durchführung des Studienvorbereitungskurses Integra 2 und beim Übergang der Kursteilnehmer in das Studium.

\section{\#talents: Digital Management-Talents Initiative}

\begin{tabular}{|c|c|}
\hline Projektleiter & Prof. Dr. rer. pol. Dana Mietzner \\
\hline Mitarbeiter & FG Innovations- und Regionalforschung \\
\hline Mittelgeber & Land Brandenburg | MWFK | ESF \\
\hline Projektvolumen & $287.660 €$ \\
\hline Laufzeit & $01 / 2016-12 / 2018$ \\
\hline Projekthomepage & www.th-wildau.de/digital-talents \\
\hline \multicolumn{2}{|c|}{$\begin{array}{l}\text { Der Aufbau digitaler Management-Kompetenzen ist für Brandenburger Unternehmen von strategischer Bedeutung. Eine } \\
\text { strukturierte Ausbildung dieser Kompetenzen an Hochschulen gibt es hier jedoch kaum. Das Vorhaben "\#talents - Digi- } \\
\text { tal Management-Talents Initiative" leistet einen Beitrag zur Schließung dieser Lücke, indem es Anforderungen an Digital } \\
\text { Management-Talents erforscht und ein Programm aufsetzt, das die Entwicklung der Digital Management-Talents an der } \\
\text { Schnittstelle zwischen Studium und Beruf zum Gegenstand hat. }\end{array}$} \\
\hline
\end{tabular}

\section{Auf Schienen: EXIST-Gründerstipendium}

\begin{tabular}{l|l}
$\begin{array}{l}\text { Projektleiter } \\
\text { Mitarbeiter }\end{array}$ & Prof. Dr. rer. pol. Dana Mietzner \\
\hline Mittelgeber & Land Brandenburg I MWFK | ESF \\
\hline Projektvolumen & $290.660 €$ \\
\hline Laufzeit & $01 / 2016-12 / 2018$ \\
\hline Projekthomepage & wWw.lokpit.com
\end{tabular}

Auf Schienen (jetzt Lokpit) standardisiert und automatisiert mit einer Software im Internet, einem dazugehörigen Dokumentationssystem und einem während des EXIST-Programms fertig zu entwickelnden Algorithmus die Vermittlung von Personal und weiterer Ressourcen zwischen Unternehmen innerhalb der Bahnbranche. Die Ergebnisse aus dem EGSProjekt sollen in ein künftiges technologieorientiertes Dienstleistungsunternehmen der Bahnbranche einfließen. 
Coaching:Pilot: Coaching Pilot für den Berufseinstieg von Studierenden im Land Brandenburg

Projektleiter

Prof. Dr. rer. pol. Dana Mietzner

Mitarbeiter

FG Innovations- und Regionalforschung

Mittelgeber Land Brandenburg | MWFK | ESF

Projektvolumen

$290.660 €$

Laufzeit

01/2016-12/2018

Projekthomepage

www.th-wildau.de/coaching-pilot

Das Projekt beinhaltet die Konzipierung, Umsetzung und Evaluierung eines neuen Coaching-Modells, um den Berufseinstieg von Studierenden zu unterstützen. Fach- und Methodenwissen sowie andere Vorkenntnisse und Erfahrungen der Teilnehmer/innen werden beim Coaching explizit berücksichtigt und an die Persönlichkeitsprofile angepasst. Parallel unterstützt das Projekt Brandenburger Unternehmen bei der Kontaktaufnahme mit zukünftigen Absolvent/innen, indem Studierende im Rahmen von Innovation Camps Konzepte zur Lösung spezifischer Innovationsprobleme entwickeln. Damit soll den spezifischen Bedarfen von kleinen und mittleren Unternehmen ebenso entsprochen werden wie den Ansprüchen einzelner Studierender. Auf diese Weise wird der Berufseinstieg sehr individuell und zielgerichtet unterstützt und Unternehmen kommen auf geeignete Art mit Hochschulen in Kontakt und können deren Transferpotenzial nutzen.

Cross.Inn: Entwicklung von labbasierten methodischen Ansätzen für die Umsetzung von

Cross-Industry-Innovation

\begin{tabular}{l|l} 
Projektleiter & Prof. Dr. rer. pol. Dana Mietzner \\
\hline Mitarbeiter & FG Innovations- und Regionalforschung \\
\hline Mittelgeber & Land Brandenburg | MWFK | EFRE | InfraFEI
\end{tabular}

Projektvolumen $\quad 99.800 €$

Laufzeit $\quad$ 02/2016-01/2017

Mit dem Vorhaben soll eine technische Infrastruktur aufgebaut werden, die das Kreativlabor der TH Wildau (ViNN:Lab) erweitert und die Umsetzung von Cross Industry-Innovation unterstützt. Weiterhin soll die Möglichkeit geschaffen werden, das noch junge Forschungsfeld der Cross Industry-Innovation durch neue methodische Ansätze zu stärken und für den Einsatz im unternehmerischen Kontext zu professionalisieren. 
EXiST-Support: Einführung eines Programms zur Etablierung einer EXiST-Kultur an der THWi

\begin{tabular}{|c|c|}
\hline Projektleiter & Prof. Dr. rer. pol. Dana Mietzner \\
\hline Mitarbeiter & FG Innovations- und Regionalforschung \\
\hline Mittelgeber & TH Wildau | Zielvereinbarung \\
\hline Projektvolumen & $55.000 €$ \\
\hline Laufzeit & 03/2017-12/2018 \\
\hline \multicolumn{2}{|c|}{$\begin{array}{l}\text { Das Vorhaben EXiST-Support hat sich zum Ziel gesetzt, die Gründungskultur hochschulintern stärker zu fundieren, Lü- } \\
\text { cken im Ablaufprozess des drittmittelgeförderten Gründungsservice zu schließen und insbesondere das EXiST-Förder- } \\
\text { programm zu verankern. Neben spezifischen Sensibilisierungsaktivitäten und Unterstützungsmaßnahmen werden ein } \\
\text { digitales Handbuch zur EXiST Administration für die Gründungsteams sowie ein „How to EXiST“ Fahrplan erstellt. Beide } \\
\text { sollen vor allem den administrativen Aufwand im Rahmen der Gründungsförderung sowohl für die Hochschule als auch } \\
\text { insbesondere für die Gründerinnen und Gründer gering halten.Darüber hinaus ist die Entwicklung eines geeigneten } \\
\text { Coaching-Ansatzes nach Gründungspersönlichkeiten vorgesehen, der auch langfristig am Zentrum für Forschung und } \\
\text { Transfer der TH Wildau zum Einsatz kommen kann und einen Beitrag leistet, die Gründungsförderung an der TH Wildau } \\
\text { zu professionalisieren. }\end{array}$} \\
\hline
\end{tabular}

\section{KIW: Mittelstand 4.0 Kompetenzzentrum IT-Wirtschaft}

Projektleiter Prof. Dr. Prer. pol. Dana Mietzner, Prof. Dr. iur. Carsten Kunkel

\begin{tabular}{|l|l|}
\hline Mitarbeiter & FG Innovations- und Regionalforschung \\
\hline Mittelgeber & Bund I BMWi \\
\hline Projektvolumen & $762.599 €$ \\
\hline
\end{tabular}

Laufzeit $\quad 12 / 2017-11 / 2020$

Das Ziel des Mittelstand-4.0-Kompetenzzentrums IT-Wirtschaft ist es, kleinen und mittleren IT-Unternehmen vor Ort bei der Bewältigung der Herausforderungen der Digitalisierung mit Expertenwissen, Demonstrationszentren, Netzwerken zum Erfahrungsaustausch und praktischen Beispielen zu helfen. Das Konsortium des Kooperationsprojektes besteht aus dem Bundesverband IT-Mittelstand e.V. (BITMi) als Konsortialführer, der Technischen Hochschule Wildau und der TH Brandenburg. Unterstützt wird der Verbund von der Wirtschaftsfördergesellschaft Berlin Partner. Im Teilprojekt „Foresight und Technologie Scouting“ der TH Wildau wird hierzu von der Forschungsgruppe Innovations- und Regionalforschung der Aufbau und Betrieb eines Foresight- und Technologie Scouting Systems für den IT Mittelstand umgesetzt. Darüber hinaus werden permanente Formate wie das „IT-Mittelstand Foresight Lab“ und das „IT-Mittelstand Innovation Camp“ angeboten. Unter der Leitung von Prof. Dr. Kunkel werden Kompetenzen aus dem Bereich rechtlicher Rahmenbedingungen in das Kompetenzzentrum eingebracht, das betrifft insbesondere die Themenbereiche Datenschutz und Vertragsrecht. Die Forschungsgruppe Innovations- und Regionalforschung ist auch für die Evaluation des Kompetenzzentrums verantwortlich. 
Local OI: Entwicklung und Testen nutzerzentrierter Innovationsmethoden

\begin{tabular}{|l|l|}
\hline Projektleiter & Prof. Dr. rer. pol. Dana Mietzner \\
\hline Mitarbeiter & FG Innovations- und Regionalforschung \\
\hline Mittelgeber & Land Brandenburg I MWFK | EFRE \\
\hline Projektvolumen & $77.360 €$ \\
\hline Laufzeit & $10 / 2016-09 / 2017$ \\
\hline
\end{tabular}

Ziel des Vorhabens ist es, eine komplexe technische und räumliche Infrastruktur aufzubauen, mit welcher der Ansatz Local Open Innovation methodisch strukturiert unterstützt und forschungsseitig begleitet werden kann. Hierbei kann das Vorhaben bereits auf sehr gute räumliche Voraussetzungen zurückgreifen, die im Rahmen der Entwicklung eines FabLabs an der TH Wildau geschaffen wurden.

Panel Bürgerwissenschaft

\begin{tabular}{|c|c|}
\hline Projektleiter & Prof. Dr. rer. pol. Dana Mietzner \\
\hline Mitarbeiter & FG Innovations- und Regionalforschung \\
\hline Mittelgeber & TH Wildau | Zielvereinbarung \\
\hline Projektvolumen & $60.000 €$ \\
\hline Laufzeit & $10 / 2016-12 / 2018$ \\
\hline Projekthomepage & www.th-wildau.de/buergerpanel \\
\hline \multicolumn{2}{|c|}{$\begin{array}{l}\text { Ziel des Vorhabens ist die Entwicklung eines Bürgerpanels für die koordinierte Einbindung potentieller Nutzer/innen in } \\
\text { Usability Tests oder als Testkundinnen und -kunden im Rahmen von Neuproduktentwicklung. Weiterhin dient das Projekt } \\
\text { der Stärkung der interdisziplinären Zusammenarbeit der Wissenschaftsbereiche der TH Wildau und der strategischen } \\
\text { Weiterentwicklung des Forschungsgebietes Innovationsmanagement. }\end{array}$} \\
\hline
\end{tabular}

\section{PHABLABS 4.0: Photonics enhanced fab labs supporting the next revolution in digitalization}

\begin{tabular}{|c|c|}
\hline Projektleiter & Prof. Dr. rer. pol. Dana Mietzner \\
\hline Mitarbeiter & FG Innovations- und Regionalforschung \\
\hline Mittelgeber & EU | Horizont 2020 | ICT \\
\hline Projektvolumen & $60.438 €$ \\
\hline Laufzeit & $12 / 2016-05 / 2019$ \\
\hline Projekthomepage & www.phablabs.eu \\
\hline \multicolumn{2}{|c|}{$\begin{array}{l}\text { Photonik ist eine Schlüsseltechnologie, die im Energie-, Gesundheits-, Produktions-, Gesundheits- und Kultursektor eine } \\
\text { essentielle Rolle spielt. Das HORIZON } 2020 \text { Projekt PHABLABS } 4.0 \text { kombiniert deshalb zwei große Trends um die Diffu- } \\
\text { sion dieser Technologie weiter zu beschleunigen. Das Projekt hat sich das Ziel gesetzt, verstärkt Aufmerksamkeit für die } \\
\text { Photonik als Innovationstreiber und Schlüsseltechnologie zu generieren und gleichzeitig die sich rasant entwickelnden } \\
\text { FabLab-Netzwerke (Fabrication Laboratories), als Orte an denen Schlüsseltechnologien praktisch von der nächsten Gene- } \\
\text { ration von Anwendenden eingesetzt werden, zu nutzen. Im Rahmen des Projektes werden vom ViNN:Lab der TH Wildau } \\
\text { in Kooperation mit dem Leibniz-Institut für Höchstfrequenztechnik und weiteren internationalen Partnern innovative DIY- } \\
\text { Photonikworkshops entwickelt und im Laufe des Projektes europaweit skaliert, getestet, iteriert und schließlich weltweit } \\
\text { allen FabLabs zur Verfügung gestellt. }\end{array}$} \\
\hline
\end{tabular}


PROKOM 4.0 - Kompetenzmanagement für die Facharbeit in der High-Tech-Industrie: Implementierung eines

Foresightprozesses und Analyse regionaler Umsetzungsbedingung

Projektleiter

Mitarbeiter

Mittelgeber

Projektvolumen

Laufzeit

Projekthomepage
Prof. Dr. rer. pol. Dana Mietzner

FG Innovations- und Regionalforschung

Bund | BMBF

$287.031 €$

01/2015-12/2017

www.prokom-4-0.de

Ziel des Verbundprojektes ist es, aufzuzeigen, welche Entwicklungen auf der Ebene der Facharbeit in welcher Form forciert werden müssen, damit der demografische Wandel, der Wandel hin zu "Industrie 4.0" und die Energiewende erfolgreich gemeistert werden können. Ziel des Teilvorhabens ist es, mögliche Zukünfte der Industrie 4.0 herauszuarbeiten, um Anforderungen an die Facharbeit und entsprechende Kompetenzen wissenschaftlich begründet ableiten zu können. In diesem Zusammenhang wird auch die These geprüft, ob und inwieweit das Konzept Industrie 4.0 neue Möglichkeiten für Cross Industry Innovation eröffnet und in welchem Maße Open Innovation-Ansätze erforderlich sind, um Kompetenzen in Unternehmen und Netzwerken aufzubauen, damit Industrie 4.0 nachhaltig implementiert werden kann. Darüber hinaus ist beabsichtigt, Foresightmethoden mit neuen Elementen anzureichern. Ein weiteres Ziel besteht in der Aufklärung der Funktion regionaler Strukturen als Entwicklungsbedingungen für die Industrie 4.0.

START LEAN - Integrierter Gründungservice an der Technischen Hochschule Wildau

\begin{tabular}{|c|c|}
\hline Projektleiter & Prof. Dr. rer. pol. Dana Mietzner \\
\hline Mitarbeiter & FG Innovations- und Regionalforschung \\
\hline Mittelgeber & Land Brandenburg | MWFK | ESF \\
\hline Projektvolumen & $447.105 €$ \\
\hline Laufzeit & $01 / 2015-12 / 2017$ \\
\hline Projekthomepage & www.th-wildau.de/gruendungsservice \\
\hline \multicolumn{2}{|c|}{$\begin{array}{l}\text { Im Rahmen des Projekts START LEAN sensibilisiert der Gründungsservice der TH Wildau Studierende, Alumni sowie Be- } \\
\text { schäftigte der Hochschule für Unternehmertum und unterstützt die Entwicklung unternehmerischer Kompetenzen. Durch } \\
\text { den Gründungsservice werden im Rahmen des Projekts individuelle, qualifizierende Coaching- und Beratungsleistungen } \\
\text { für Gründungs- und Nachfolgeinteressierte u. a. in den Bereichen Ideengenerierung, Geschäftsmodellierung, Finanzie- } \\
\text { rung, Marketing sowie Vertrieb angeboten. }\end{array}$} \\
\hline
\end{tabular}

\section{ViNN:Lab: Erhöhung von Performance \& Impact}

\begin{tabular}{l|l}
\hline Projektleiter & Prof. Dr. rer. pol. Dana Mietzner \\
\hline Mittelgeber & TH Wildau | Zielvereinbarung \\
\hline Projektvolumen & $120.000 €$ \\
\hline Laufzeit & $10 / 2016-12 / 2018$ \\
\hline
\end{tabular}

Das ViNN:Lab erfüllt als Kreativ- und Prototypinglabor an der TH Wildau die Funktion eines Wissensintermediärs. Ziel des Vorhabens „Impact \& Performance“ ist es daher, bestehende Potentiale in den Bereichen der Nutzerinteraktion und -reichweite besser zu nutzen und dadurch die hohe regionale und überregionale Ausstrahlungskraft des ViNN:Labs, die Integration in die Lehre sowie die Nutzung im Rahmen interdisziplinärer Forschung weiter zu verbessern. 
ViTeP - Virtuelle und interdisziplinäre Team- und Projektarbeit

Projektleiter Prof. Dr. rer. pol. Dana Mietzner

Mitarbeiter FG Innovations- und Regionalforschung

Mittelgeber TH Wildau | Zielvereinbarung

Projektvolumen $54.190 €$

Laufzeit 03/2017-12/2018

Der Zuwachs projektförmiger \& virtueller (Team)Arbeit, die steigende Bedeutsamkeit des routinierten Umgangs mit digitalen Technologien und Medien sowie die Notwendigkeit ausgeprägter Soft Skills sind nicht nur Treiber neuer Arbeitsformen, sondern auch Strukturgeber für die Kompetenz- und Anforderungsprofile von Arbeitnehmenden der Zukunft. Vor diesem Hintergrund soll ViTeP als fächergruppenübergreifendes Modul an der TH Wildau etabliert werden, um Studierende der Fachbereiche Wirtschaft, Informatik, Recht (WIR) und Ingenieur- und Naturwissenschaften (INW) umfassender auf das Arbeiten in einer digitalen Arbeitswelt vorzubereiten, die durch die Zusammenarbeit in virtuellen und interdisziplinären Teams gekennzeichnet ist. Darüber hinaus werden nach der Pilotierungsphase des ViTeP-Moduls Transferworkshop für alle interessierten Einzelpersonen und Teams der TH Wildau konzeptioniert und durchgeführt.

Studienstart 2.0 - mobile Beratung für beruflich Qualifizierte

\begin{tabular}{|l|l|}
\hline Projektleiter & Dr. Andreas Preiß \\
\hline Mittelgeber & Land Brandenburg \\
\hline Projektvolumen & $212.434 €$ \\
\hline Laufzeit & $05 / 2015-02 / 2018$ \\
\hline
\end{tabular}

Mit diesem Projekt wird der geringen Studienneigung beruflich Qualifizierter entgegengewirkt. Für diese äußerst heterogene Zielgruppe ist es nicht leicht, den Weg an die Hochschule zu finden. Als eine Ursache lassen sich Informationsdefizite identifizieren. Bedenkt man darüber hinaus die vergleichsweise höheren beruflichen- und/oder familiären Verpflichtungen beruflich Qualifizierter, zielt das Projekt auf die Erprobung passgenauer, mobiler Beratungsmöglichkeiten.

beMINT: beMINT. Berufsperspektiven zum Anfassen

\begin{tabular}{l|l}
\hline $\begin{array}{l}\text { Projektleiter } \\
\text { Mittelgeber }\end{array}$ & Dr. Anke Renger \\
\hline IHK Berlin \\
\hline $\begin{array}{l}\text { Projektvolumen } \\
\text { Laufzeit }\end{array}$ & $33.797 €$ \\
\hline $03 / 2017-11 / 2018$ \\
\hline $\begin{array}{l}\text { Das Kooperationsprojekt beMINT ermöglicht erstmalig die effektive Verzahnung von verschiedenen Aspekten der Berufs- } \\
\text { wahl. Neben Begeisterung und Interesse für Naturwissenschaft und Technik zu wecken, stehen praktisches Experimentie- } \\
\text { ren, Beratung zu Ausbildungsberufen und Karrieremöglichkeiten, Berufsorientierung „vor Ort“ im Fokus. NaWiTex gehört } \\
\text { dabei zum Verbundpartner Berlin-Brandenburger Netzwerk der Schülerlabore GenaU. }\end{array}$
\end{tabular}


DAAD-Promos: PROMOS-Mobilitätsprogramm 2017

\begin{tabular}{l|l}
$\begin{array}{l}\text { Projektleiter } \\
\text { Mittelgeber }\end{array}$ & Dr. phil. Angelika Schubert \\
\hline $\begin{array}{l}\text { Projektvolumen } \\
\text { DAAD }\end{array}$ & $11.000 €$ \\
\hline $\begin{array}{l}\text { Laufzeit } \\
01 / 2017-12 / 2017\end{array}$ \\
$\begin{array}{l}\text { Das Promos-Projekt dient der Förderung der Mobilität deutscher Studierender ins außereuropäische Ausland. Die TH } \\
\text { Wildau fördert hauptsächlich Studienaufenthalte, insbesondere an unsere Partner-HS (z.B. in China und Australien), Prak- } \\
\text { tika sowie Auslandsvorhaben in Vorbereitung von Abschlussarbeiten. }\end{array}$
\end{tabular}

\section{DAAD-Stibet: STIBET sowie DAAD-Preis 2017}

\begin{tabular}{l|l} 
Projektleiter & Dr. phil. Angelika Schubert
\end{tabular}

\section{Mittelgeber DAAD}

\begin{tabular}{l|l} 
Projektvolumen & $21.397 €$
\end{tabular}
Laufzeit
01/2017-12/2017

Das Stibet-Projekt dient der Förderung von Betreuungsmaßnahmen für ausländische Studierende der TH Wildau. Zudem sollen Kontaktstipendien und Studienabschlussbeihilfe für internationale Studierende vergeben werden. Darüber hinaus ist auch die Vergabe des DAAD-Preises, dotiert mit $1.000 €$, für eine/n herausragende/n internat. Studierende/n geplant.

\section{Erasmus+ / Projekt 2017: Erasmus+ Mobilität mit Programmländern der EU}

\begin{tabular}{l|l}
\hline $\begin{array}{l}\text { Projektleiter } \\
\text { Mittelgeber }\end{array}$ & Dr. phil. Angelika Schubert \\
\hline Projektvolumen & $66.220 €$ \\
\hline Laufzeit & $06 / 2017-05 / 2019$
\end{tabular}

Das Erasmus+ Projekt der EU soll Studienaufenthalte von Studierenden beider Fachbereiche sowie Kurzlehraufenthalte von Dozenten an europäischen Partner-HS fördern und damit die Mobilität steigern. Ferner werden auch Weiterbildungsund Trainingsmaßnahmen in Europa für Kollegen und Mitarbeiter finanziell unterstützt, um die Internationalisierung weiter zu fördern.

\section{AQUSIS - Ausbau der Qualifizierungs- und Unterstützungsangebote für internationale Studierende}

\begin{tabular}{l|l}
\hline Projektleiter & Rouven Sperling, M. A. \\
\hline Mittelgeber & Land Brandenburg | MWFK | ESF \\
\hline Projektvolumen & $177.654 €$ \\
\hline Laufzeit & $05 / 2015-04 / 2018$
\end{tabular}

Das Projekt hat das Ziel, ausländische Studierende zu fördern, um ihre Integrationsfähigkeit zu erhöhen. Dabei wird die Strategie der TH Wildau hinsichtlich der Eingliederung von ausländischen Studierenden in den Brandenburger Arbeitsmarkt und dementsprechende Angebote weiterentwickelt. Es wird an Ansätze und Erfahrungen angeknüpft, die in dem Projekt Integration ausländischer Hochschulabsolventinnen und Hochschulabsolventen in den regionalen Arbeitsmarkt bereits erarbeitet wurden. 
A\&W Welcome Center: Ausbau und Weiterentwicklung der Angebote des Welcome Centers der TH Wildau

Projektleiter Prof. Dr. rer. nat. Ulrike Tippe

Mittelgeber Land Brandenburg I MWFK

Projektvolumen $37.000 €$

Laufzeit

01/2017-12/2017

Das Welcome Center der TH Wildau konzipiert und organisiert seit 2016 Studienvorbereitungskurse (Sprachkurse und propädeutische Kurse) für Geflüchtete. Mit diesem Projekt wird die Aufrechterhaltung dieses Angebots im Jahr 2017 unterstützt.

\section{A\&W Welcome Center 2: Ausbau und Weiterentwicklung der Angebote des Welcome Centers der TH Wildau}

Projektleiter Prof. Dr. rer. nat. Ulrike Tippe

Mittelgeber Land Brandenburg I MWFK

Projektvolumen $50.000 €$

Laufzeit

01/2017-12/2017

Das Welcome Center der TH Wildau konzipiert und organisiert seit 2016 Studienvorbereitungskurse (Sprachkurse und propädeutische Kurse) für Geflüchtete. Mit diesem Projekt wird die Aufrechterhaltung dieses Angebots im Jahr 2017 unterstützt.

\section{Profilgebundene WissensChecks}

Projektleiter

Prof. Nikola Budilov-Nettelmann

Mittelgeber Land Brandenburg | MWFK | ESF

Projektvolumen $66.916 €$

Laufzeit $01 / 2016-06 / 2018$

Das Projekts ist ein Verbundprojekt der BTU Cottbus-Senftenberg, der TH Wildau, der Hochschule für nachhaltige Entwicklung Eberswalde und der TH Brandenburg. Profilgebunde WissensChecks sollen an der TH Wildau in MINT-Fächern erstellt und allen Studieninteressierten für die Entscheidung über die Studienwahl und zur Unterstützung des Studienvortschritts in der Studieneingangsphase zur Verfügung gestellt werden. 
Projektleiter

Mittelgeber

Projektvolumen

Laufzeit
Prof. Nikola Budilov-Nettelmann

Bund | BMBF

$1.086 .271 €$

$10 / 2016-12 / 2020$

In der ersten Förderperiode wurden vom Projekt SOS - Strukturierung und Optimierung des Selbststudiums Online-Kursräume und E-Assessments für Mathematik und Rechnungswesen entwickelt, um die Leistungen der Studierenden zu verbessern. In Mathematik war es dafür notwendig, zunächst die Vorbereitungsangebote neu zu konzipieren und im Blended-Learning-Format umzusetzen. In beiden Fachrichtungen wurde allen Studierenden eine tutorielle Unterstützung angeboten. Für E-Assessments wurden die technischen und strukturellen Voraussetzungen geschaffen sowie ein Fragenpool mit insgesamt über 1800 Fragen aufgebaut. Ziele für die zweite Förderperiode sind der Ausbau des Unterstützungsangebots in Mathematik (erweitert auf Statistik) durch passgenauere, anwendungsbezogene Online-Angebote und Beratung; die überfachliche Unterstützung des Selbststudiums; der Aufbau eines hochschulweiten Präsenz- und Online-Tutoriensystems; die systematische Heranführung der Lehrenden aller Fachgebiete an qualitätsgesicherte E-Assessments. Die Mathematikmaterialien und E-Assessments sollen für mobile Endgeräte angepasst und zusätzlich in englischer Sprache angeboten werden. Ein Fokus liegt dabei auf der nachhaltigen Einbindung in die Lehre durch Kooperation mit Lehrenden und Struktureinheiten der Hochschule.

\section{TH College: Studienvorbereitung und Studienverlauf - Aufbau des TH Colleges}

\begin{tabular}{|c|c|}
\hline Projektleiter & Prof. Dr. rer. nat. Ulrike Tippe \\
\hline Mittelgeber & Land Brandenburg | MWFK | ESF \\
\hline Projektvolumen & $411.137 €$ \\
\hline Laufzeit & $01 / 2016-12 / 2018$ \\
\hline \multicolumn{2}{|c|}{$\begin{array}{l}\text { Durch den Ausbau der Studienvorbereitung und Studienbegleitung sowie durch Einrichtung einer Koordination zur Ver- } \\
\text { netzung aller Aktivitäten in den Bereichen Studienorientierung, Studienvorbereitung und studienbegleitung hat das Pro- } \\
\text { jekt die Steigerung der Studierfähigkeit und die Erhöhung des Studienerfolgs insbesondere von beruflich Qualifierten, } \\
\text { Berufstätigen und Menschen mit Migrationshintergrund zum Ziel. }\end{array}$} \\
\hline
\end{tabular}

Technologietransferstelle der TH Wildau

\begin{tabular}{l|l} 
Projektleiter Dipl.-Ingenieur (FH) Annette Vossel & D
\end{tabular}

Mitarbeiter Christine Richert, Stefanie Radig

Mittelgeber Land Brandenburg | MWE | EFRE

\begin{tabular}{l|l} 
Projektvolumen & $158.427 €$
\end{tabular}

Laufzeit $\quad$ 01/2017-12/2017

Projekthomepage www.th-wildau.de/transfer

Die Technologietransferstelle ist die zentrale Schnittstelle zwischen der Hochschule und (Brandenburger) Unternehmen. Ziel ist es zum einen, den Technologiebedarf von Unternehmen, insbesondere kleinen und mittleren Unternehmen (KMU), aufzunehmen und ihn durch Forschung und Entwicklungs (FuE)-Lösungen der Hochschule zu befriedigen. Zum anderen können über die Technologietranfserstelle FuE-Ergebnisse aus der Hochschule in die Wirtschaft getragen und dort zu neuen Produkten entwickelt werden. Die Unternehmen (speziell die KMU) sollen für die Möglichkeiten und Chancen von FuE-Projekten mit der Technischen Hochschule Wildau aber auch anderen Hochschulen und Forschungseinrichtungen im Land Brandenburg sensibilisiert werden. 
BIDS 2015- 2017 - Betreuungsinitiative Deutsche Auslands- und Partnerschulen

\begin{tabular}{l|l}
$\begin{array}{l}\text { Projektleiter } \\
\text { Mittelgeber }\end{array}$ & Dipl.-Wirtsch.infor. (FH) Carolina Winkler \\
\hline $\begin{array}{l}\text { Projektvolumen } \\
\text { DAAD }\end{array}$ & $90.000 €$ \\
\hline Laufzeit & $02 / 2015-12 / 2017$ \\
\hline $\begin{array}{l}\text { Seit dem Jahr 2002 verfolgt die TH Wildau die Strategie, gezielt Kooperationen mit Deutschen Schulen in Lateinamerika } \\
\text { aufzubauen. Diese wurden 2008-2010 im Rahmen des BIDS-Programms vom DAAD gefördert, so dass erhebliche Qua- } \\
\text { litäts- und Quantitätssprünge in der Betreuung und Akquise lateinamerikanischer Studenten erreicht wurden. Zur Zeit } \\
\text { bestehen Kooperationen mit } 16 \text { Schulen in Lateinamerika. }\end{array}$
\end{tabular}




\section{TABELLARISCHE ÜBERSICHT PUBLIKATIONEN}

Althaus M (2017)

Althaus M (2017)

Althaus M (2017)

Althaus M (2017)

Althaus M (2017)

Althaus M (2017)

Althaus M (2017)

Baetge D (2017)

Baetge D (2017)

Baetge D (2017)

Baetge D (2017)

Baetge D (2017)
Die Medien im Wahlkampf. Bewährungsprobe für die Vermittler in der Demokratie. In: Peter Lang GmbH, Frankfurt am Main, ISBN: 978-3-63173531-2

Evaluation und lose Enden. Leistungsbewertung im Public Affairs Management. In: [Nachname nicht vorhanden] $\mathrm{MH}((\mathrm{ed})$ Anwendungsgebiete und Grundlagen Strategischer Kommunikation. Nomos, BadenBaden, pp 58-109

Grundsätze der Politikberatung für die kommunale Jugendlobby. In: Lindner W, Pletzer W (eds) Kommunale Jugendpolitik. Beltz Juventa, Weinheim, ISBN: 978-3-7799-3463-9, pp 252-269

Lobby-Register: Juristische Hausaufgaben gemacht. PR Magazin 42(3):19

Lobby-Register: Vorleistung für den Koalitionspoker. PR Magazin 42(4):17

Politikberatung und Lobbying. In: Ministerium für Familie Frauen Jugend Integration und Verbraucherschutz, Landesamt für Soziales Jugend und Versorgung, Rheinland-Pfalz (eds) PEP-Dokumentation: Praxisentwicklungsprojekt zur Profilierung von Jugendarbeit in Rheinland-Pfalz. Rheinland-Pfalz, Ministerium für Familie, Frauen, Jugend, Integration und Verbraucherschutz, Mainz, pp 24-31

Trumps Wahlkampf: Rebellion gegen die Profis der Politikberatungsindustrie? In: Rodenberg H-P (ed) Trump: Politik als Geschäft. Lit Aktuell 9. Lit, Berlin Münster, ISBN: 978-3-643-13789-0, pp 145-166

Kommentierung von Art. 35 CISG (Vertragsmäßigkeit der Ware). In: Würdinger M, Herberger M, Martinek M, Rüßmann H, Weth S (eds) Juris Praxiskommentar BGB, 8th. Juris, Saarbrücken, ISBN: 978-3-86330-161-3, Rn. 1-54

Kommentierung von Art. 36 CISG (Maßgeblicher Zeitpunkt für die Vertragsmäßigkeit). In: Würdinger M, Herberger M, Martinek M, Rüßmann $\mathrm{H}$, Weth S (eds) Juris Praxiskommentar BGB, 8th. Juris, Saarbrücken, ISBN: 978-3-86330-161-3, Rn. 1-17

Kommentierung von Art. 37 CISG (Nacherfüllung bei vorzeitiger Lieferung). In: Würdinger $M$, Herberger $M$, Martinek $M$, Rüßmann $H$, Weth S (eds) Juris Praxiskommentar BGB, 8th. Juris, Saarbrücken, ISBN: 978-386330-161-3, Rn. 1-17

Kommentierung von Art. 38 CISG (Untersuchung der Ware). In: Würdinger M, Herberger M, Martinek M, Rüßmann H, Weth S (eds) Juris Praxiskommentar BGB, 8th. Juris, Saarbrücken, ISBN: 978-3-86330-161-3, Rn. 1-49

Kommentierung von Art. 39 CISG (Mängelrüge). In: Würdinger M, Herberger M, Martinek M, Rüßmann H, Weth S (eds) Juris Praxiskommentar BGB, 8th. Juris, Saarbrücken, ISBN: 978-3-86330-161-3, Rn. 1-43 
Kommentierung von Art. 40 CISG (Bösgläubigkeit des Verkäufers). In: Würdinger M, Herberger M, Martinek M, Rüßmann H, Weth S (eds) Juris Praxiskommentar BGB, 8th. Juris, Saarbrücken, ISBN: 978-3-86330-161-3, Rn. 1-17

Baetge D (2017)

Kommentierung von Art. 41 CISG (Rechtsmängel). In: Würdinger $\mathrm{M}$, Herberger M, Martinek M, Rüßmann H, Weth S (eds) Juris Praxiskommentar BGB, 8th. Juris, Saarbrücken, ISBN: 978-3-86330-161-3, Rn. 1-26

Baetge D (2017)

Kommentierung von Art. 42 CISG (Belastung mit Schutzrechten Dritter). In: Würdinger M, Herberger M, Martinek M, Rüßmann H, Weth S (eds) Juris Praxiskommentar BGB, 8th. Juris, Saarbrücken, ISBN: 978-3-86330161-3, Rn. 1-23

Baetge D (2017)

Kommentierung von Art. 43 CISG (Rügepflicht). In: Würdinger M, Herberger M, Martinek M, Rüßmann H, Weth S (eds) Juris Praxiskommentar BGB, 8th. Juris, Saarbrücken, ISBN: 978-3-86330-161-3, Rn. 1-22

Baetge D (2017)

Kommentierung von Art. 44 CISG (Entschuldigung für unterlassene Anzeige). In: Würdinger M, Herberger M, Martinek M, Rüßmann H, Weth $\mathrm{S}$ (eds) Juris Praxiskommentar BGB, 8th. Juris, Saarbrücken, ISBN: 978-386330-161-3, Rn. 1-18

Baetge D (2017)

Kommentierung von Art. 5 EGBGB (Personalstatut). In: Würdinger M, Herberger M, Martinek M, Rüßmann H, Weth S (eds) Juris Praxiskommentar BGB, 8th. Juris, Saarbrücken, ISBN: 978-3-86330-161-3, Rn. 1-91

Kommentierung von Art. 6 EGBGB (Öffentliche Ordnung, ordre public).

Baetge D (2017) In: Würdinger M, Herberger M, Martinek M, Rüßmann H, Weth S (eds) Juris Praxiskommentar BGB, 8th. Juris, Saarbrücken, ISBN: 978-3-86330161-3, Rn. 1-142

Baetge D (2017)

Kommentierung von Art. 78 CISG (Zinsen). In: Würdinger M, Herberger M, Martinek M, Rüßmann H, Weth S (eds) Juris Praxiskommentar BGB, 8th. Juris, Saarbrücken, ISBN: 978-3-86330-161-3, Rn. 1-25

Kommentierung von Art. 79 CISG (Hinderungsgrund außerhalb des Einflussbereichs des Schuldners). In: Würdinger $M$, Herberger $M$, Baetge D (2017) Martinek M, Rüßmann H, Weth S (eds) Juris Praxiskommentar BGB, 8th. Juris, Saarbrücken, ISBN: 978-3-86330-161-3, Rn. 1-33

Kommentierung von Art. 80 CISG (Verursachung der Nichterfüllung durch die andere Partei). In: Würdinger $M$, Herberger $M$, Martinek M, Rüßmann H, Weth S (eds) Juris Praxiskommentar BGB, 8th. Juris, Saarbrücken, ISBN: 978-3-86330-161-3, Rn. 1-17

Agile Methoden als Moderatoren zur Steuerung der sozialen Beschleunigung. Wissenschaftliche Beiträge 21:83-88. doi: 10.15771/09498214_2017_11

Beyond Zeitmanagement - Zeitmanagement mit agilen Methoden. InHaack B (2017) formationstechnologie und Immobilien - Magazin für IT-Anwendungen in der Wohnungswirtschaft 24: 11-13

Haack B, Tippe U, Stobernack M, Wendler T (2017)

Mathematik für Wirtschaftswissenschaftler. Springer Gabler, Berlin Heidelberg. ISBN: 978-3-642-55174-1. doi: 10.1007/978-3-642-55175-8 
Haas S (2017)

Haas S, Jeremias XV, Rabe C (2017)

Hartmann F (2017)

Hartmann F, Hartmann V (2017)

Hartmann F, Mietzner D (2017)

Hartmann F, Mietzner D (2017)

Hasenmüller M, Seeliger F (2017)

Höppner T (2017)

Höppner T (2017)

Höppner T (2017)

Höppner T (2017)

Höppner T (2017)

Höppner T (2017)
Abo-Commerce-Modelle in Deutschland: Eine inhaltsanalytische Untersuchung. In: Deutscher Dialogmarketing Verband (ed) Dialogmarketing Perspektiven 2016/2017. Springer Fachmedien Wiesbaden, Wiesbaden, ISBN: 978-3-658-16835-3, pp 73-85. doi: 10.1007/978-3-658-16835-3_4

Formative E-Assessments als Unterstützung zum strukturierten Selbststudium: Ergebnisse eines Lehrexperiments im Studiengang Europäisches Management (BA). Wissenschaftliche Beiträge 21: 89-99. doi: 10.15771/0949-8214_2017_12

Anforderungen an Kompetenzen im Zusammenhang mit Industrie 4.0 - Eine Bestandsaufnahme. In: Facharbeit und Digitalisierung. Ergebnisse aus dem BMBF-Verbundprojekt „Kompetenzmanagement für die Facharbeit in der High-Tech Industrie. PROKOM 4.0, Bottrop, Duisburg, Erkrath, Flensburg, Rheine, Rostock, Wildau, ISBN: 978-3-9810056-9-1, pp 19-28

Digitalisierung und Industrie 4.0. In: Facharbeit und Digitalisierung. Ergebnisse aus dem BMBF-Verbundprojekt „Kompetenzmanagement für die Facharbeit in der High-Tech Industrie. PROKOM 4.0, Bottrop, Duisburg, Erkrath, Flensburg, Rheine, Rostock, Wildau, ISBN: 978-3-98100569-1, pp 8-14

Industrie 4.0 und die Maker Bewegung? - Ein Perspektivwechsel. In: Facharbeit und Digitalisierung. Ergebnisse aus dem BMBF-Verbundprojekt „Kompetenzmanagement für die Facharbeit in der High-Tech Industrie. PROKOM 4.0, Bottrop, Duisburg, Erkrath, Flensburg, Rheine, Rostock, Wildau, ISBN: 978-3-9810056-9-1, pp 29-35

The Maker Movement - Current Understanding and Effects on Production. The Proceedings of The XXV ISPIM Conference 2017. In: ISBN: 978 952-335-021-2

Der Kunde allein im Haus: Mit der personallosen Öffnung zur 24/7Bibliothek? Ein Überblick über Open-Library-Konzepte. BuB : Forum für Bibliothek und Information 69(2-3): 82-84

Duty to Treat Downstream Rivals Equally: (Merely) a Natural Remedy to Google's Monopoly Leveraging Abuse. European Competition and Regulatory Law Review (CoRe) 1(3):208-221

EU copyright reform: the case for a related right for press publishers. In: CREATe Public Lecture Series 2017. CREATe. doi: 10.5281/zenodo.495480

EU vs. Google: Nur was heißt „Gleichbehandlung“ im Internet? Kommunikation \& Recht (K\&R) 24(9): Editorial

Google Search (Shopping): Etablierte Prinzipien für digitalen Präzedenzfall. Wirtschaft und Wettbewerb (WuW) 67(9): Editorial

Neue EU-Richtlinie zum Leistungsschutzrecht. In: FIW Heft 262 - Jahrbuch 2016/2017. Carl Heymanns Verlag, Köln, ISBN: 978-3-452-29073-1, pp 79-85

The proposed Directive on Copyright in the Digital Single Market (Articles 11, 14 and 116). Strengthening the Press Through Copyright. doi: $10.2861 / 104262$ 
Wegweisend: Die EU-Entscheidung gegen Google. In: 31. Jahrbuch des BDZV 'Zeitungen 2017/2018‘. ZV Zeitungs-Verlag Service GmbH, Berlin, ISBN: 978-3-939705-22-2, pp 151-164

Höppner T, Kretschmer M, Xalabarder R (2017)

Höppner T, Schaper F (2017)

Kunkel C (2017)

Kunkel C (2017)

Kunkel C (2017)

Kunkel C (2017)

Kunkel C, Kunkel O (2017)

Kunkel C, Kunkel O (2017)

Kunkel C, Kunkel O (2017)

Kunkel C, Kunkel O (2017)

Kunkel C, Kunkel O (2017)

Kunkel C, Kunkel O (2017)
CREATe public lectures on the proposed EU right for press publishers. European Intellectual Property Review (EIPR) 39(4): 607-622

CREATe public lectures on the proposed EU right for press publishers. European Intellectual Property Review (EIPR) 39(4): 607-622

Alle Jahre wieder: Beitragspflicht aufgrund IHK-Pflichtmitgliedschaft zur Verfassungsmäßigkeit von Pflichtmitgliedschaften in Kammern und der damit verbunden Beitragserhebung. Anmerkung zu Bayerischer Verwaltungsgerichtshof, Beschluss vom 20.09.2016 - 22 ZB 16.288. Juris-PraxisReport Handels- und Gesellschaftsrecht 9(7): Anm. 4

Vertragsgestaltung bei der $\mathrm{GmbH}$, 1st edn. Kautelarjuristische Schriftenreihe zum Wirtschaftsrecht. epubli, Berlin. ISBN: 978-3-7450-4748-6

Wirtschaftsrecht, 2nd edn. Schriftenreihe des Privaten Instituts für Angewandtes Wirtschaftsrecht. epubli, Berlin. ISBN: 978-3-7450-4704-2

Zur Bedeutung der Kautelarjurisprudenz im Wirtschaftsrecht, insbesondere im GmbH-Recht. In: Kunkel C (ed) Vertragsgestaltung bei der $\mathrm{GmbH}, 1$ st edn. Kautelarjuristische Schriftenreihe zum Wirtschaftsrecht. epubli, Berlin, ISBN: 978-3-7450-4748-6, pp 1-18

Gerichtliche Überprüfung der Wirksamkeit von Gesellschafterbeschlüssen bei Abberufung oder Kündigung eines Anstellungsvertrags eines Gesellschafter-Geschäftsführers einer $\mathrm{GmbH}$ aus wichtigem Grund. Anmerkung zu BGH, 2. Zivilsenat, Urteil, 04.04.2017, II ZR 77/16. JurisPraxisReport Handels- und Gesellschaftsrecht 9(6): Anmerkung 2

Gesellschaftsrecht I, 3rd edn. Schriftenreihe des Privaten Instituts für Angewandtes Wirtschaftsrecht. epubli, Berlin. ISBN: 978-3-7450-1381-8

Gesellschaftsrecht II, 3rd edn. Schriftenreihe des Privaten Instituts für Angewandtes Wirtschaftsrecht. epubli, Berlin. ISBN: 978-3-7450-1382-5

Haftung des Gesellschafter-Geschäftsführers einer Ein-PersonenGesellschaft. Anmerkung zu LG München II, Urteil vom 26.01.2017, 3 O 3420/15. juris PraxisReport Strafrecht 11(20): Anmerkung 2

Insiderhandel und Marktmanipulation nach der Neufassung des WpHG. Anmerkung zu BGH 5. Strafsenat, Beschluss vom 10. Januar 2017, Az: 5 StR 532/16. juris PraxisReport Compliance \& Investigations 4(3): Anmerkung 4

Kein Schutz vor strafrechtlicher Haftung durch bloße Einführung eines Compliance Management Systems. Anmerkung zu BGH, Urteil vom 06.09.2016, 1 StR 104/15. juris PraxisReport Compliance \& Investigations 4(2): Anmerkung 3 
Die Kunst der Vertragsverhandlung - Spieltheorie einmal anders. In: Jahrbuch der Rechtsdidaktik 2016. Yearbook of Legal Education. BWV Berliner Wissenschafts-Verlag GmbH, Berlin, ISBN: 978-3-8305-3775-5, pp 235-258

Vertragsverhandlung und Spieltheorie, 1st edn. Kautelarjuristische Schriftenreihe zum Wirtschaftsrecht, Band 2. epubli, Berlin. ISBN: 9783745074963

Meyer S (2017)

Gleichgeschlechtliche Ehe unabhängig vom Ehebegriff des Art. 6 Abs. 1 GG verfassungsmäßig. Zeitschrift für das gesamte Familienrecht (FamRZ) 64(16): $1281-1284$

Meyer S (2017)

Kriminalwissenschaftliche Prognoseinstrumente im Tatbestand polizeilicher Vorfeldbefugnisse. Die Zukunft der Terrorismusbekämpfung im Lichte des BKA-Urteils. JuristenZeitung 72(9): 429-439

Meyer S (2017)

Subjektiver oder objektiver Gefahrenbegriff, „Gefahrenverdacht“ und Vorfeldbefugnisse: Dauerbaustellen des Gefahrenabwehrrechts. JURA Juristische Ausbildung 39(11): 1259-1270

Meyer S (2017)

The EU and Nanotechnologies. A Critical Analysis. By Tanja Ehnert. European Journal of Risk Regulation (EJRR): 804-810

Mietzner D, Hartmann F, Fahrenkrug M, Fahrenkrug K (2017)

Mietzner D, Lahr M (2017)

Schultz C, Mietzner D (2017)

Tschiedel R, Hartmann F (2017)
Strategische Vorausschau bei der Multiwatt ${ }^{\circledast} \mathrm{GmbH}$. Wissenschaftliche Beiträge 21:101-108. doi: 10.15771/0949-8214_2017_13

Think, Make, Share - Die Rolle von Makerspaces an Hochschulen. Synergie: Fachmagazin für Digitalisierung in der Lehre 4:24-27

Anforderungen an das Teammatching aus der Sicht von hochschulnahen Gründerteams. In: Mieke C, Siemon C (eds) Gründung und Innovation, Band 6. Gründung und Innovation. Logos Verlag, Berlin, ISBN: 978-3-8325-4430-0, pp 165-185

Innovationsorientierende Technikfolgenabschätzung zur Erarbeitung von Handlungsoptionen für kleine und mittelständische Unternehmen. In: Bornewasser M (ed) Vernetztes Kompetenzmanagement. Springer Berlin Heidelberg, Berlin, Heidelberg, ISBN: 978-3-662-54953-7, pp 123-133. doi: 10.1007/978-3-662-54954-4_10

\section{FORSCHUNGSPREISE}

Prof. Dr. rer. pol. Dana Mietzner

Prof. Dr. rer. nat. Margit Scholl
Forschungsprofessur 2012-2017 der TH Wildau

Forschungsprofessur 2014-2019 der TH Wildau
„Innovations- und Regionalmanagement "

"Ganzheitlicher Aufbau und partizipatives Management von Smart-Technologien des 21. Jahrhunderts“ 